CONFORMAL GEOMETRY AND DYNAMICS

An Electronic Journal of the American Mathematical Society

Volume 7, Pages 103-151 (September 9, 2003)

S $1088-4173(03) 00065-1$

\title{
CUSPS IN COMPLEX BOUNDARIES OF ONE-DIMENSIONAL TEICHMÜLLER SPACE
}

\author{
HIDEKI MIYACHI
}

Dedicated to my father Kenji Miyachi for his 60th Birthday

\begin{abstract}
This paper gives a concrete proof of the conjectural phenomena on the complex boundary one-dimensional slices: every rational boundary point is cusp shaped. This paper treats this problem for Bers slices, the Earle slice, and the Maskit slice. In proving this, we also show that every Teichmüller modular transformation acting on a Bers slice can be extended as a quasiconformal mapping on its ambient space. Furthermore, using this extension, we discuss similarity phenomena on the boundaries of Bers slices, and also compare these phenomena with results in complex dynamics. We will also give a result, related to the theory of L. Keen and C. Series, of pleated varieties in quasifuchsian space of once punctured tori.
\end{abstract}

\section{Contents}

1. Introduction 104

2. Preliminaries $\quad 109$

3. Complex length and end invariants $\quad 113$

4. Trace functions and the geometry of parameter spaces 114

5. Pleating rays 118

6. Geometry on the boundaries of convex cores 118

7. Derivative is of linear order 124

8. Differential on a pinched curve under qc-deformation 129

9. Differential on remaining curves under qc-deformation $\quad 130$

10. Non-vanishing theorem for the derivative of trace functions 132

11. Teichmüller modular group acting on Bers slices $\quad 139$

12. Extended local pleating theorem

Appendix A. Relation to complex dynamics $\quad 147$

References 148

Received by the editors July 2, 2000 and, in revised form, February 20, 2003.

2000 Mathematics Subject Classification. Primary 30F40, 30F60; Secondary 37F30, 37F45.

Key words and phrases. Kleinian group, Teichmüller theory, deformation space.

This work was done when the author was partially supported by Research Fellowships of the Japan Society for the Promotion of Science for Young Scientists.

(C)2003 American Mathematical Society 


\section{INTRODUCTION}

1.1. Problem. This paper concerns the shapes of deformation spaces for once punctured torus groups:

Problem. How is the shape of quasifuchsian space described as the subset of the space of representations?

By virtue of Bers' simultaneous uniformization theorem, quasifuchsian space is a domain in the space of representations of the fundamental group of a once punctured torus, and is biholomorphically equivalent to the product of two Teichmüller spaces (of mutually orientation reversed surfaces). On the other hand, C.McMullen [44] observed that the closure of the quasifuchsian space is NOT a topological submanifold with boundary in the space of representations (see also [22]), which implies the boundary of this space seems to be complicated. Meanwhile, many one-dimensional slices of quasifuchsian space are drawn as the computer pictures, and also have complicated boundaries. Indeed, C.McMullen [44] also gave an interesting picture of a slice of quasifuchsian space. In the picture, one can find many points where two slices touch.

In this paper, we focus on the shapes of the boundaries of three kinds of slices: Bers slices, the Earle slice, and the Maskit slice (see Figures 1, 2, and 3). Each of these slices is realized as the image of the canonical embedding from Teichmüller space of once punctured tori, and is deeply related to the moduli of Kleinian groups. The boundaries of their slices are called the complex boundaries. This terminology is adopted after Lipman Bers. Indeed, he used this terminology to indicate the boundary of his slices ([7]).

Recently, Y.N.Minsky [47] gave an affirmative answer on the ending lamination conjecture for once punctured torus groups. Thanks to this beautiful work, one can see that each of the three embeddings can be extended homeomorphically to Thurston's compactification of Teichmüller space of once punctured tori with the property that, except at most two laminations, every rational lamination in the Thurston boundary corresponds to a geometrically finite group containing the accidental parabolic transformation associated with the lamination (cf. [47] and 29]). In this paper, such boundary points of slices are called rational boundary points.

The central subject of the paper is to study the shape of the complex boundaries of the above three slices near rational boundary points. We will give a concrete proof of the folklore conjecture:

Theorem. Every rational boundary point is cusp shaped.

Cusp shapes as universal phenomena. D. Mumford and D. Wright drew the computer graphic of the Maskit slice a few decades ago. Recently, pictures of a Bers slice and the Earle slice were drawn by P. Liepa and Y. Yamashita, respectively. Pictures of linear slices, that is, slices defined by fixing some trace function, had been given by C. McMullen and D. Wright. Pictures of other one-dimensional parameter spaces, though not for once punctured torus groups, were given by R. Riley, F. Gehring and G. Martin, and D. Wright. These pictures also seem to admit cusps in their boundaries like those in our three slices. Results given in this paper support the conjectural phenomena at the rational boundary points of these slices (see [18], [31], [44], [45], [53], 63], 64], and 665]). 


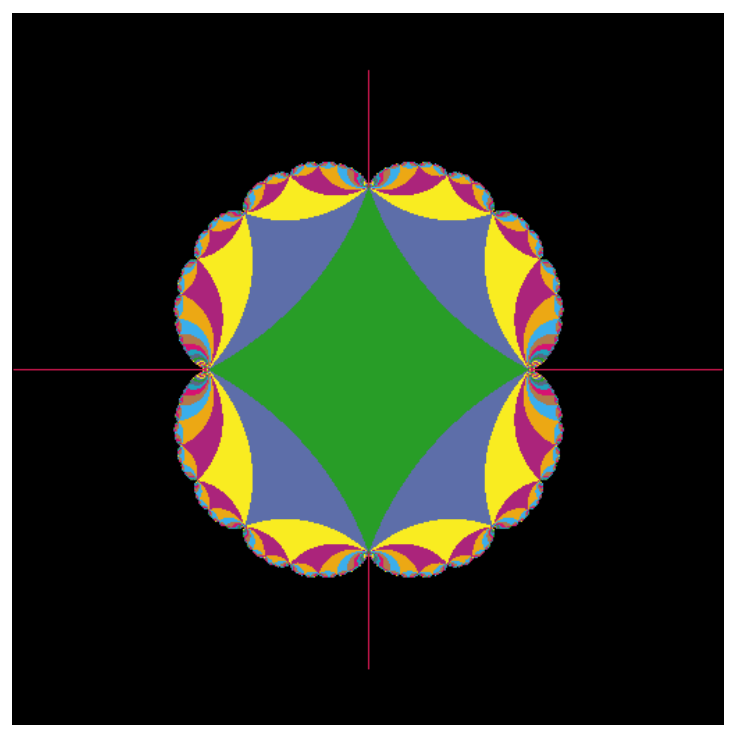

Figure 1. A Bers slice: Courtesy of Yasushi Yamashita

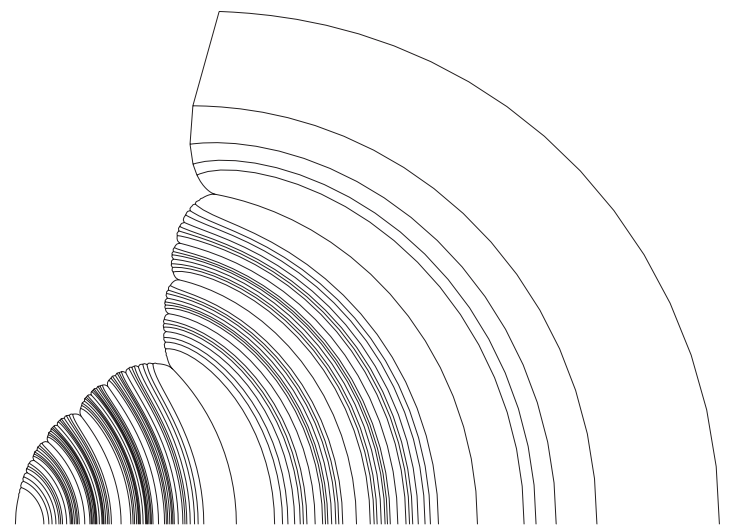

Figure 2. The upper half of the Earle slice: Courtesy of Peter Liepa

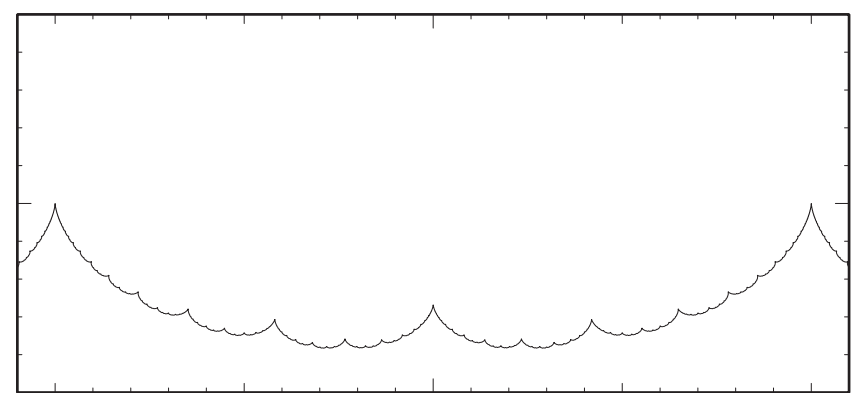

Figure 3. The Maskit slice: Courtesy of David J. Wright 
1.2. Statement of results of this paper. As noted in the previous section, the main purpose of this paper is to prove the folklore conjecture:

Theorem 1 (Rational boundary points are cusps). Every rational boundary point of three slices is a $(2,3)$-cusp.

The definition of a $(2,3)$-cusp is given in Section 2 As a corollary, we have

Corollary 1. All three slices are not quasidisks.

Corollary 1 for the case of the Maskit slice has already been observed in the author's earlier paper [48] (see also Corollary 7).

Theorem 1 is related to D. Wright's conjecture in 63: In the late 1980's, D. Mumford, C. McMullen, and D. Wright drew the computer graphical picture of the Maskit embedding and found many cusps on its boundary. In drawing this picture, Wright conjectured that, in the case of the Maskit embedding, every rational boundary point is a simple zero of corresponding trace function, while he found that this conjecture implies an affirmative answer for the folklore conjecture in the case of the Maskit embedding. Actually, Theorem 1 follows from the next theorem, which contains the affirmative answer to Wright's conjecture:

Theorem 2 (Rational boundary points are simple zeros). For each of the three slices, every rational boundary point is a simple zero of the trace function corresponding to its accidental parabolic.

We also show the following theorem.

Theorem 3 (Modular transformation has qc-extension). Every Teichmüller modular transformation acting on a Bers slice admits a quasiconformal extension to the ambient space. Furthermore, the maximal dilatation of an extension is dominated by the maximal dilatation of the extremal quasiconformal self-mapping acting on the base surface of the Bers slice, which is homotopic to the homeomorphism corresponding to the given modular transformation.

\subsection{Applications of results.}

1.3.1. Extreme points for outer radius. As an immediate corollary of Theorem 1 one can determine the class of groups to be extreme points of outer radius of Bers slices. The outer radius of a Bers slice is an important notion from the function theoretic point of view and extreme points for the outer radius often have some extremal properties like the Köebe function (cf. [36]).

Corollary 2. Any extreme point of the outer radius of a Bers slice corresponds to a totally degenerate group.

Indeed, it can be observed from Euclidean geometry that the outer radius cannot be attained at any $(2,3)$-cusp.

1.3.2. Similarity phenomena on Bers boundaries. The following problem was posed by C. McMullen (cf. p.178 of [43]).

Problem. Is the boundary of a Bers slice self-similar about the fixed points of every hyperbolic modular transformation?

Theorem 3 gives one answer for McMullen's problem. 
Corollary 3. The closure of a Bers slice is weakly quasiconformally self-similar about the fixed points of any hyperbolic modular transformation.

In this paper, a set $E \subset \hat{\mathbb{C}}$ is called weakly quasiconformally self-similar about $e_{0} \in E$ if there exists a quasiconformal mapping $f$ of $\hat{\mathbb{C}}$ and at most one point $e_{1} \in E \backslash\left\{e_{0}\right\}$ satisfying the following:

(1) $f(E)=E, f\left(e_{0}\right)=e_{0}$, and $\bar{\partial} f \equiv 0$ on the interior of $E$.

(2) For any neighborhood $U$ of $e_{0}$ in $\hat{\mathbb{C}} \backslash\left\{e_{1}\right\}, E \backslash\left\{e_{1}\right\}=\bigcup_{n \in \mathbb{N}} f^{n}(U \cap E)$ holds.

In Section 11 we also observe the following similarity phenomenon among cusps in the boundary of a Bers slice:

Corollary 4 (All rational boundary points are similar to each other). Every Teichmüller modular transformation acting on a Bers slice is $C^{1+\alpha}$-conformal at all rational boundary points, where $\alpha>0$ depends only on the base point of a given Bers slice, independent of the choice of elements of modular transformation.

In Appendix $\mathrm{A}$ we will discuss similarity phenomena regarding the boundary of the Mandelbrot set as related to this corollary.

1.3.3. Hölder continuity at boundary. Combining Theorem 3 and the Hölder continuity of quasiconformal mappings (see p.70 of [37]), we also obtain a refinement of a result of Bers (see p.51 of [7]):

Corollary 5. Every Teichmüller modular transformation acting on a Bers slice can be extended Hölder-continuously on the closure of the Bers slice.

1.3.4. Extended local pleating theorem. We extend the local pleating theorem due to L. Keen and C. Series to a neighborhood of geometrically finite boundary groups (cf. 26] and Section [12). The once punctured torus group [ $\eta]$ is called a boundary group if it is Kleinian but not quasifuchsian]. If a boundary group is geometrically finite, it has either one or two conjugacy classes of primitive accidental parabolics. In this paper, a geometrically finite boundary group is of type $n$ if it admits $n$ conjugacy classes of primitive accidental parabolics.

Theorem 4 (Extended local pleating theorem: the rational case). For any geometrically finite boundary group $\left[\eta_{0}\right]$, there exists a neighborhood $\mathcal{U}$ of $\left[\eta_{0}\right]$ in the space of representations which satisfies the following property: Suppose that $\left[\eta_{0}\right]$ is of type $n$, and let $\left\{\mathbf{w}_{i}\right\}_{1 \leq i \leq n}$ be elements of the fundamental group of a once punctured torus such that each $\eta_{0}\left(\mathbf{w}_{i}\right)$ is a primitive accidental parabolic. For $[\eta] \in \mathcal{U}$, if $\operatorname{tr}^{2} \eta\left(\mathbf{w}_{i}\right)$ are real and greater than 4 , then $[\eta]$ is a quasifuchsian representation. Furthermore, the convex core of the Kleinian manifold associated with $[\eta]$ is bent along the geodesic corresponding to $\eta\left(\mathbf{w}_{i}\right)(1 \leq i \leq n)$.

Theorem 4 is related to the following result due to J. Otal in [51:

Theorem (Otal). Let $\eta$ be a Kleinian once punctured torus group. Let $\mathbf{w}$ be an element of the fundamental group of a once punctured torus which represents a simple closed curve. If $\eta(\mathbf{w})$ is hyperbolic and if its translation length is less than some universal constant, then the boundary of the convex core of the associated Kleinian manifold is bent along the geodesic corresponding to $\eta(\mathbf{w})$.

\footnotetext{
${ }^{1}$ By virtue of Minsky's theorem, boundary groups can be characterized by this property in the case of once punctured torus groups.
} 
Our theorem is proved without using Otal's result. The resemblance between ours and Otal's is that both theorems are concerned with the behavior of the pleating locus of the convex core in the case when the hyperbolic element corresponding to the locus is sufficiently close to parabolic. There are two differences: The first is an advantage of ours. In Otal's case, a given group for which it is to be ascertained whether the associate convex core is bent or not, is assumed to be discrete at the beginning. This is not so in our case. Furthermore, our theorem gives a decision as to whether the group is discrete. The second is a disadvantage of ours. In our case, we are looking only near geometrically finite groups, while Otal's theorem treats any once punctured torus group and points out the universality for the condition of translation length.

1.4. Outline of the paper. In Section 2, we give the notation and definitions used in this paper. The definition of Minsky's end invariants and the statement of his pivot theorem (a special case) are reviewed in Section 3.

Section 4 treats a relation between the shape of the complex boundary near a rational boundary point and the trace function associated with a given rational boundary point. In this section we will show that Theorem 2 implies Theorem 1 (see Proposition 4.3). The most crucial reason why rational boundary points are cusp shaped is that, when groups move along complex boundaries converging to the group of a given rational boundary point, the suitable branch of the complex length of the corresponding trace function converges to zero along a path running parallel to a circle which is contained in the right-half plane and tangent to zero. This phenomenon is completely analyzed by using Minsky's pivot theorem.

In the proof of Theorem [2] the theory of pleating coordinates occupies an important portion (26] and [44]). In Sections 5 and 6, we review notation concerning geometries and moduli of the convex core of Kleinian manifolds. For example, the definitions and basic properties of pleating rays, $F$-peripheral disks and subgroups are given in these sections.

In Section 7, Theorem 9, which is the most important theorem for the proof of Theorem 2, is stated. Theorem 9 tells us that along a pleating ray, the derivative of the complex length of a trace function not associating with the bending locus is dominated by a linear function of the length of the bending locus. In Section 7.3 we define quasiconformal deformations of groups on pleating rays for treating the derivative of trace functions. In Section 7.4, we state two theorems, Theorem 11 and 12 which imply Theorem 9 We prove Theorem 9 in Section 7.4 . We prove Theorems 11 and 12] in Sections 8 and 9, respectively. In Section [10] one can see how Theorem 9 contributes to the proof of Theorem 2 , independent of the proof of Theorem 9

In Section 11, we will prove Theorem 3. The soul of the proof is to recognize quasifuchsian space and the cotangent bundle over Teichmüller space as the total spaces of the holomorphic families of Bers slices and their ambient spaces over the Teichmüller space respectively. Applying the global triviality of the cotangent bundle, we can understand that Bers slices move holomorphically in $\mathbb{C}$ when their base surfaces also move complex analytically. Together with this observation, the proof is obtained by applying the Improved $\lambda$-lemma and the Bers' marking trick in [7] (that is, a diagonal action of the mapping class group on quasifuchsian space). One can read this section independently of the other results. 
We prove Theorem 4 in Section 12 To prove this theorem we will define a local coordinate near a geometrically finite boundary group in the space of representations using associated trace functions.

In Appendix A, we will give a brief explanation about a relation between our result and the complex dynamics, and add a column in Sullivan's dictionary.

Notation. $A \asymp B$ and $A \lesssim B$ mean $A / C<B<C A$ and $A<C B$ for some implicit constant $C$, respectively. For $z, w \in \mathbb{H}$ and $c>0, z \approx_{c} w$ means that the hyperbolic distance between $z$ and $w$ is bounded by $c$.

\section{Preliminaries}

2.1. (2,3)-cusps. Let $a, r>0$. Define

$$
C^{+}(a, r)=\left\{z=x+i y \in \mathbb{C}|| z \mid<r, y^{2}>a x^{3}, x \geq 0\right\} \cup\{|z|<r, x<0\}
$$

and

$$
C^{-}(a, r)=\left\{z=x+i y \in \mathbb{C}|| z \mid<r, y^{2}<a x^{3} x \geq 0\right\} .
$$

Let $E$ be a domain in $\mathbb{C}$ and $\zeta_{0} \in \partial E \cap \mathbb{C}$. We say that $\zeta_{0}$ is a $(2,3)$-cusp of $E$ if there exist $a>b>0, r>0$, and $\theta \in \mathbb{R}$ such that

$$
\zeta_{0}+e^{\theta} \cdot C^{+}(a, r) \subset E \quad \text { and } \quad\left\{\zeta_{0}+e^{\theta} \cdot C^{-}(b, r)\right\} \cap \bar{E}=\emptyset .
$$

See Figure 4. A typical example of a $(2,3)$-cusp is the cusp of the cardioid $\left\{z^{2}|| z-1 \mid<1\right\}$.

2.2. Enumeration of simple closed curves and Teichmüller space. Throughout this paper, we denote by $\Sigma$ an oriented once punctured torus and by $\pi_{1}$ the fundamental group of $\Sigma$. For $\mathbf{a}^{\prime}, \mathbf{a}^{\prime \prime} \in \pi_{1}$, the product $\mathbf{a}^{\prime} \mathbf{a}^{\prime \prime}$ means the homotopy class of a curve which first passes along a curve in $\mathbf{a}^{\prime \prime}$ and next passes along a curve in $\mathbf{a}^{\prime}$.

2.2.1. Enumeration of simple closed curves. An ordered pair $(\mathbf{a}, \mathbf{b})$ of generators of $\pi_{1}$ is called canonical if the algebraic intersection number of $\mathbf{a}$ and $\mathbf{b}$ with respect to the given orientation of $\Sigma$ is equal to +1 . The commutator $[\mathbf{a}, \mathbf{b}]=\mathbf{a}^{-1} \mathbf{b}^{-1} \mathbf{a b}$ represents a loop around the puncture. Henceforth, we fix a canonical generator pair $(\mathbf{a}, \mathbf{b})$ of $\pi_{1}$.

We review an identification between the homotopy classes of all simple closed curves on $\Sigma$ and $\hat{\mathbb{Q}}=\mathbb{Q} \cup\{1 / 0\}$. Henceforth, we will always express rational numbers in the form $p / q$ where $p$ and $q$ are relatively prime integers and $q>0$.

Set $\mathbf{w}(1 / 0)=\mathbf{a}^{-1}$ and $\mathbf{w}(n / 1)=\mathbf{a}^{-n} \mathbf{b}(n \in \mathbb{Z})$. For $p / q, p^{\prime} / q^{\prime} \in \hat{\mathbb{Q}}$ with $p q^{\prime}-p^{\prime} q=-1$, we define $\mathbf{w}\left(\left(p+p^{\prime}\right) /\left(q+q^{\prime}\right)\right)=\mathbf{w}\left(p^{\prime} / q^{\prime}\right) \mathbf{w}(p / q)$. Then, $\mathbf{w}(p / q) \in \pi_{1}$ can be defined for all $p / q \in \hat{\mathbb{Q}}$ and the homology class of a simple closed curve in $\mathbf{w}(p / q)$ is equal to that in $\mathbf{a}^{-p} \mathbf{b}^{q}$. See for instance, [24], [30, [50], and [63].

2.2.2. Teichmüller space and modular transformations. The Teichmüller space of $\Sigma$ is a set of equivalence classes of a pair $(f, R)$ of an orientation preserving homeomorphism $f$ of $\Sigma$ to an analytically finite Riemann surface $R$, where two pairs $(f, R),\left(f^{\prime}, R^{\prime}\right)$ are equivalent if there exists a conformal mapping $h$ from $R$ onto $R^{\prime}$ which is homotopic to $f^{\prime} \circ f^{-1}$. The Teichmüller distance is defined by

$$
d_{\text {Teich }(\Sigma)}\left((f, R),\left(f^{\prime}, R^{\prime}\right)\right)=\log \inf _{h} K(h)
$$




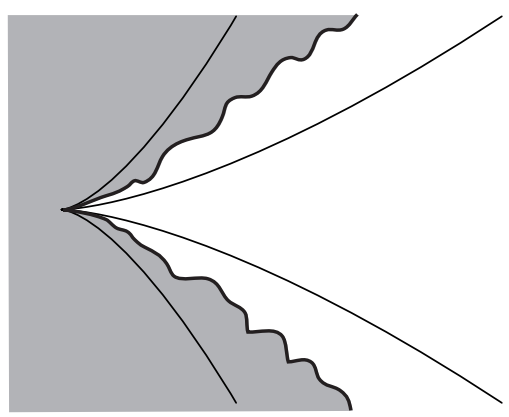

Figure 4. A (2,3)-cusp

where $h$ runs over quasiconformal mappings $h$ of $R$ to $R^{\prime}$ which are homotopic to $f^{\prime} \circ f^{-1}$ and $K(h)$ is the maximal dilation of $h$. This infimum is always attained by the unique extremal quasiconformal mapping (cf. 21]).

A canonical generator $(\mathbf{a}, \mathbf{b})$ on $\Sigma$ determines an identification between Teichmüller space of $\Sigma$ and the upper-half plane $\mathbb{H}$ via their periods. Under this identification, Teichmüller space with the Teichmüller distance is isometric to the hyperbolic 2-plane $\mathbb{H}$ (of curvature -1 ).

Let $\operatorname{Mod}(\Sigma)$ be the mapping class group of $\Sigma$. Any element $[\omega] \in \operatorname{Mod}(\Sigma)$ induces the isomorphism $[\omega]_{*}$, called Teichmüller modular transformation, of Teich $(\Sigma)$ by the equation

$$
[\omega]_{*}((f, S))=\left(f \circ \omega^{-1}, S\right) .
$$

$[\omega] \in \operatorname{Mod}(\Sigma)$ determines the element $[\bar{\omega}] \in \operatorname{Mod}(\bar{\Sigma})$ by conjugating by the canonical orientation reversing involution. This satisfies that

$$
[\bar{\omega}]_{*}(y)=\overline{[\omega]_{*}(\bar{y})} \quad \text { for all } y \in \operatorname{Teich}(\bar{\Sigma}) .
$$

2.3. Once punctured torus groups. We give the definitions of once punctured torus groups, and the space of representations.

2.3.1. Space of representations $\mathcal{R}$. A homomorphism from $\pi_{1}$ to $\mathrm{PSL}_{2}(\mathbb{C})$ is called admissible if it sends the commutator $[\mathbf{a}, \mathbf{b}]$ to a parabolic transformation. Denote by $\mathcal{R}$ the space of admissible representations of $\pi_{1}$ into $\mathrm{PSL}_{2}(\mathbb{C})$. The space $\mathcal{R}$ is a two-dimensional complex analytic space such that every irreducible representation corresponds to a regular point, and the trace function of $\mathbf{w} \in \pi_{1}$ :

$$
\mathcal{R} \ni[\eta] \mapsto \operatorname{tr}^{2} \eta(\mathbf{w}) \in \mathbb{C}
$$

is holomorphic.

2.3.2. Once punctured torus groups. An admissible homomorphism (or its $\mathrm{PSL}_{2}(\mathbb{C})$ conjugacy class) is said to be a once punctured torus group if it is a conjugacy class of a discrete and faithful representation; by abuse of language, such a representation itself is called by the same terminology. Let $\eta$ be a once punctured torus group with invariant component. An invariant component $\Delta$ of $\eta$ is called distinguished if $\eta$ induces an orientation preserving homeomorphism from $\Sigma$ onto $\Delta / \eta\left(\pi_{1}\right)$.

Let $\mathcal{D}$ be the set of all once punctured torus groups and $\mathcal{Q F}$ the subset of $\mathcal{D}$ which consists of all quasifuchsian representations. $\mathcal{Q} \mathcal{F}$ is called quasifuchsian space. Y. Minsky proved that the closure of $\mathcal{Q F}$ coincides with $\mathcal{D}$ ([47]). 
Quasifuchsian space has the product structure as follows:

Henceforth, for any 3-manifold $M$ with boundary, the following orientation convention is applied to $\partial M$ : A frame $f$ on $\partial M$ is positive if $(f, n)$ is positive with respect to the orientation of $M$ where $n$ is an inward-pointing vector on $\partial M$. When $M$ is a Kleinian 3-manifold, the orientation on $\partial M$ coincides with that inherited from the Riemann sphere.

Let $\bar{\Sigma}$ denote $\Sigma$ with the orientation reversed. Let $x=(f, S) \in \operatorname{Teich}(\Sigma)$ and $y=(g, R) \in \operatorname{Teich}(\bar{\Sigma})$. Bers' simultaneous uniformization tells us that $x$ and $y$ determine a marked quasifuchsian group $G(x, y)$ (up to conjugation in $\mathrm{PSL}_{2}(\mathbb{C})$ ) such that the quasifuchsian manifold $\left(\mathbb{H}^{3} \cup \Omega(x, y)\right) / G(x, y)$ is bounded by Riemann surfaces $R$ and $S$, where $\Omega(x, y)$ means the region of discontinuity of $G(x, y)$. Furthermore, there exists a homeomorphism between $\Sigma \times[0,1]$ to $\left(\mathbb{H}^{3} \cup \Omega(x, y)\right) / G(x, y)$, compatible with corresponding markings and orientations between their boundaries. Let $\eta_{G(x, y)}$ be the representation associated with the marked quasifuchsian group $G(x, y)$. Then Bers showed that

$$
\operatorname{Teich}(\Sigma) \times \operatorname{Teich}(\bar{\Sigma}) \ni(x, y) \mapsto\left[\eta_{G(x, y)}\right] \in \mathcal{Q F}
$$

is biholomorphic.

2.4. Definitions of slices. This section gives the definitions of three slices: a Bers slice, the Maskit slice, and the Earle slice.

2.4.1. The Bers embedding $\mathcal{B}_{G}$. In this paper, we treat a Bers slice in a somehow extended sense from the usual one (cf. e.g. [21]).

Fix a pair $\left(z_{0}, y_{0}\right) \in \operatorname{Teich}(\Sigma) \times \operatorname{Teich}(\bar{\Sigma})$. Let $\eta_{G\left(z_{0}, y_{0}\right)}$ be a representation associated with $G\left(z_{0}, y_{0}\right)$ as in the previous section. We normalize this representation so that its limit set contains three points, 0,1 , and $\infty$. Denote by $\Omega_{+}\left(z_{0}, y_{0}\right)$ the distinguished invariant component of $G\left(z_{0}, y_{0}\right)$ and, by $\Omega_{-}\left(z_{0}, y_{0}\right)$, the other component.

Let $(x, y) \in \operatorname{Teich}(\Sigma) \times \operatorname{Teich}(\bar{\Sigma})$; there exists a quasiconformal mapping $W_{y}^{x}$ which fixes the three points $\{0,1, \infty\}$ such that the conjugation $W_{y}^{x} G\left(z_{0}, y_{0}\right)\left(W_{y}^{x}\right)^{-1}$ is a quasifuchsian group and

$$
\left[\eta_{G(x, y)}\right]=\left[\mathbf{a}^{\prime} \mapsto W_{y}^{x} \circ \eta_{G\left(z_{0}, y_{0}\right)}\left(\mathbf{a}^{\prime}\right) \circ\left(W_{y}^{x}\right)^{-1}\right] .
$$

In the case of $y=y_{0}$, we may assume that $f_{y_{0}}^{x, z_{0}}:=W_{y_{0}}^{x} \circ\left(W_{y_{0}}^{z_{0}}\right)^{-1}$ is conformal on $\Omega_{-}\left(z_{0}, y_{0}\right)$. Let $Q_{-4}\left(z_{0}, y_{0}\right)$ be the complex Banach space of automorphic forms of weight -4 with respect to $G\left(x_{0}, y_{0}\right)$ with the hyperbolic $L^{\infty}$-norm. The Bers embedding $\beta_{y_{0}}^{z_{0}}$ (with respect to $G\left(z_{0}, y_{0}\right)$ ) is the mapping from Teich $(\Sigma)$ into $Q_{-4}\left(z_{0}, y_{0}\right)$ defined by

$$
\beta_{y_{0}}^{z_{0}}(x)=\mathcal{S}\left(f_{y_{0}}^{x, z_{0}}\right)
$$

where $\mathcal{S}(-)$ means the Schwarzian derivative. One can see that $\beta_{y_{0}}^{z_{0}}$ is a holomorphic embedding and whose image $\mathcal{B}_{G\left(z_{0}, y_{0}\right)}$ is a bounded domain in $Q_{-4}\left(z_{0}, y_{0}\right)$. The image is called the Bers slice with respect to $G\left(z_{0}, y_{0}\right)$.

Let $G=G\left(z_{0}, y_{0}\right)$. Let us introduce the following notation for convenience of later arguments: Let $\varphi \in \mathcal{B}_{G}$ and $x \in \operatorname{Teich}(\Sigma)$ so that $\varphi=\beta_{y_{0}}^{z_{0}}(x)$. Then, we put?

$$
\rho_{\varphi}^{b}(-)=W_{y_{0}}^{x} \circ \eta_{G\left(z_{0}, y_{0}\right)}(-) \circ\left(W_{y_{0}}^{x}\right)^{-1}
$$

\footnotetext{
${ }^{2}$ The superscript "b" comes from the name "Bers slice".
} 
and

$$
\Omega_{\varphi}^{b}=W_{y_{0}}^{x}\left(\Omega_{+}\left(z_{0}, y_{0}\right)\right) .
$$

It is known that, for $p / q \in \mathbb{Q}$, there exists the unique point $x^{b}(p / q) \in \partial \mathcal{B}_{G}$ such that the image of $\rho_{x^{b}(p / q)}^{b}$ is a regular b-group with one primitive accidental parabolic transformation $\rho_{x^{b}(p / q)}^{b}(\mathbf{w}(p / q))$. A boundary point $\varphi$ of $\mathcal{B}_{G}$ corresponds to a geometrically finite group if and only if $\varphi=x^{b}(p / q)$ for some $p / q \in \mathbb{Q}$. The set $\left\{x^{b}(p / q)\right\}_{p / q \in \hat{\mathbb{Q}}}$ are nothing but the rational boundary points in $\partial \mathcal{B}_{G}$.

The Bers slice $\mathcal{B}_{G}$ can be recognized as a slice of holomorphic mapping from $Q_{-4}\left(z_{0}, y_{0}\right)$ to $\mathcal{R}$ as follows: Let $\varphi \in Q_{-4}\left(z_{0}, y_{0}\right)$. With the Schwarzian equation $\mathcal{S}(-)=\varphi$, the monodromy homomorphism $\rho_{\varphi}^{b}: \pi_{1} \rightarrow \mathrm{PSL}_{2}(\mathbb{C})$ is associated (see e.g. 20]). Then, the Bers slice $\mathcal{B}_{G}$ is the component of the preimage of quasifuchsian space under the holomorphic mapping

$$
Q_{-4}\left(z_{0}, y_{0}\right) \ni \varphi \mapsto\left[\rho_{\varphi}^{b}\right] \in \mathcal{R}
$$

containing the origin (cf. [57]).

When we need to care about where the discussing differential lies, for $\varphi \in$ $Q_{-4}\left(z_{0}, y_{0}\right)$, we will denote by $\rho_{\varphi,\left(z_{0}, y_{0}\right)}^{b}$ instead of $\rho_{\varphi}^{b}$. This notation will be used in the proof of Proposition 10.3 of Section 10 to show our main theorem in the case of Bers slices.

2.4.2. The Earle embedding $\mathcal{E}$. Most of facts in this subsection can be referred to in Komori and Series' paper [30].

For $d \in \mathbb{C}^{*}$, we define a homomorphism $\rho_{d}^{e}$ from $\pi_{1}$ to $\mathrm{PSL}_{2}(\mathbb{C})$ by

$$
\rho_{d}^{e}(\mathbf{a})=\left[\begin{array}{cc}
\frac{d^{2}+1}{d} & \frac{d^{3}}{2 d^{2}+1} \\
\frac{2 d^{2}+1}{d} & d
\end{array}\right], \quad \rho_{d}^{e}(\mathbf{b})=\left[\begin{array}{cc}
\frac{d^{2}+1}{d} & -\frac{d^{3}}{2 d^{2}+1} \\
-\frac{2 d^{2}+1}{d} & d
\end{array}\right] .
$$

The domain $\mathcal{E}$ in $\mathbb{C}$ is defined by

$$
\mathcal{E}=\left\{d \in \mathbb{C} \mid \operatorname{Re} d>0, \quad\left[\rho_{d}^{e}\right] \in \mathcal{Q F}\right\} .
$$

Denote by $\Omega_{d}^{e}$ the distinguished invariant component of $\rho_{d}^{e}\left(\pi_{1}\right)$. Then as in the case of the Bers embedding, we obtain the isomorphism, called the Earle embedding, from Teich $(\Sigma)$ onto $\mathcal{E}$. We know that $\rho_{d}^{e}\left(\pi_{1}\right)$ has an elliptic involution $z \mapsto-z$ which gives the conjugation between $\rho_{d}^{e}(\mathbf{a})$ and $\rho_{d}^{e}(\mathbf{b})$. Thus, the Earle embedding represents a point of Teich $(\Sigma)$ by a quasifuchsian group with suitable elliptic involution (cf. 13] and [34]). The domain $\mathcal{E}$ is invariant under the complex conjugation $d \mapsto \bar{d}$. One can imagine the shape of the whole $\mathcal{E}$ by taking the reflection of P. Liepa's figure along its bottom break.

The above elliptic involution induces the relation $\operatorname{tr}^{2} \rho_{d}^{e}(\mathbf{w}(p / q)) \equiv \operatorname{tr}^{2} \rho_{d}^{e}(\mathbf{w}(q / p))$ on $\mathbb{C}^{*}$. It is known that for every $p / q \in \hat{\mathbb{Q}} \backslash\{ \pm 1 / 1\}$, there exists the unique point $x^{e}(p / q)$ in $\partial \mathcal{E}$ such that the image of $\rho_{x^{e}(p / q)}^{e}$ is a maximally parabolic group with parabolic transformations $\rho_{x^{e}(p / q)}^{e}(\mathbf{w}(p / q)), \rho_{x^{e}(p / q)}^{e}(\mathbf{w}(q / p))$, and $\rho_{x^{e}(p / q)}^{e}([\mathbf{a}, \mathbf{b}])$, and $\operatorname{Im} x^{e}(p / q)>0$ if $-1<p / q<1$. By a maximally parabolic group we mean a Kleinian group with the largest number of the non-conjugate rank 1 maximal parabolic subgroup (see [27]).

The boundary point $d$ of $\mathcal{E}$ corresponds to a geometrically finite group if and only if $d=x^{e}(p / q)$ for some $p / q \in \hat{\mathbb{Q}} \backslash\{ \pm 1 / 1\}$. Notice that $x^{e}(p / q)$ and $x^{e}(q / p)$ 
are different. In fact, it holds that $x^{e}(q / p)=\overline{x^{e}(p / q)}$ (the complex conjugation). As in the case of the Bers embedding, the rational boundary points of $\mathcal{E}$ are $\left\{x^{e}(p / q)\right\}_{p / q \in \hat{\mathbb{Q}}-\{ \pm 1 / 1\}}$.

2.4.3. The Maskit embedding $\mathcal{M}$. We deal with the Maskit embedding via the socalled horocyclic coordinate (see [33]). The horocyclic coordinate is defined as follows: For $\mu \in \mathbb{C}$, we define the representation $\rho_{\mu}^{m}$ of $\pi_{1}$ by

$$
\rho_{\mu}^{m}(\mathbf{a})=\left[\begin{array}{ll}
1 & 2 \\
0 & 1
\end{array}\right], \quad \rho_{\mu}^{m}(\mathbf{b})=\left[\begin{array}{cc}
-i \mu & -i \\
-i & 0
\end{array}\right] .
$$

Then the domain $\mathcal{M}$ is defined as the component of the interior of the set

$$
\left\{\mu \in \mathbb{C} \mid\left[\rho_{\mu}^{m}\right] \in \mathcal{D}\right\}
$$

which contains $4 i$. It is known that for each $\mu \in \mathcal{M}, \rho_{\mu}^{m}\left(\pi_{1}\right)$ is a terminal regular b-group of type $(1,1)$.

Denote by $\Omega_{\mu}^{m}, \mu \in \mathcal{M}$, the invariant component of $\rho_{\mu}^{m}\left(\pi_{1}\right)$. Then we can define the biholomorphic mapping, called the horocyclic coordinate, of Teich $(\Sigma)$ onto $\mathcal{M}$ as in the subsections above. This mapping is often referred to as the Maskit embedding of $\operatorname{Teich}(\Sigma)$ (cf. [32]).

We find the unique point $x^{m}(p / q) \in \partial \mathcal{M} \backslash\{\infty\}$ for $p / q \in \mathbb{Q}$ satisfying that $\rho_{x^{m}(p / q)}^{m}\left(\pi_{1}\right)$ is a maximal parabolic group whose parabolic transformations are $\rho_{x^{m}(p / q)}^{m}(\mathbf{w}(p / q)), \rho_{x^{m}(p / q)}^{m}(\mathbf{w}(1 / 0))$, and $\rho_{x^{m}(p / q)}^{m}([\mathbf{a}, \mathbf{b}])$. Further, it is known that $\mu \in \partial \mathcal{M}$ is a rational boundary point if and only if $\mu=x^{m}(p / q)$ for some $p / q \in \mathbb{Q}$ (see [24]).

Notation. To simplify the arguments, we put

$$
\mathbb{Q}_{\mathcal{X}}= \begin{cases}\hat{\mathbb{Q}} & \text { if } \mathcal{X}=\mathcal{B}_{G}, \\ \hat{\mathbb{Q}}-\{ \pm 1 / 1\} & \text { if } \mathcal{X}=\mathcal{E}, \text { or } \\ \mathbb{Q} & \text { if } \mathcal{X}=\mathcal{M} .\end{cases}
$$

Let us denote by $\left(\mathcal{X}_{0}, \mathcal{X}\right)$ and $\left\{\eta_{x}\right\}_{x \in \mathcal{X}_{0}}$ the pair and the holomorphic family of admissible representations corresponding to one of the following three cases:

(B) $\mathcal{X}_{0}=Q_{-4}\left(G\left(z_{0}, y_{0}\right)\right), \mathcal{X}=\mathcal{B}_{G\left(z_{0}, y_{0}\right)}$, and $\eta_{x}=\rho_{x}^{b}$.

(E) $\mathcal{X}_{0}=\mathbb{C}^{*}, \mathcal{X}=\mathcal{E}$, and $\eta_{x}=\rho_{x}^{e}$.

(M) $\mathcal{X}_{0}=\mathbb{C}, \mathcal{X}=\mathcal{M}$, and $\eta_{x}=\rho_{x}^{m}$.

Denote by $G_{x}, x \in \mathcal{X}$ the image of $\pi_{1}$ under $\eta_{x}$. For $p / q \in \mathbb{Q} \mathcal{X}$, let $x(p / q)$ be the rational boundary point of $\mathcal{X}$ corresponding to the geometrically finite group containing $X_{p / q, x}:=\eta_{x}(\mathbf{w}(p / q))$ as an accidental parabolic transformation, and in the case of the Earle slice, with the additional condition that $\operatorname{Im} x(p / q)>0$ if $-1<p / q<1$ and $x(q / p)=\overline{x(p / q)}$.

\section{Complex length And End invariants}

According to Minsky's work [47, every once punctured torus group is determined by its end invariants. We recall here the definition of end invariants of once punctured torus groups and a relation between end invariants of a given group and complex length of loxodromic elements in the group.

Let $\eta$ be a once punctured torus group. According to Bonahon's fundamental work [10], $\eta$ induces an orientation preserving homeomorphism of $\Sigma \times(-1,1)$ onto the associated manifold $\mathbb{H}^{3} / \eta\left(\pi_{1}\right)$ and the orientation of $\Sigma$ agrees with that induced 
from $\Sigma \times\{1\}$. Let us denote by $e_{+}$the end of $\mathbb{H}^{3} / \eta\left(\pi_{1}\right)$ corresponding to $\Sigma \times\{1\}$ and, by $e_{-}$, the other end.

The region of discontinuity of $\eta\left(\pi_{1}\right)$ is divided into two disjoint pieces $\Omega_{+}$and $\Omega_{-}$ corresponding to $e_{+}$and $e_{-}$, respectively. (One (or both) of them may be empty.) There are three possibilities for each of these boundaries, corresponding to three types of end invariants (let $s$ denote either + or - ):

- $\Omega_{s}$ is a topological disk, and $\Omega_{s} / \eta\left(\pi_{1}\right)$ is a once punctured torus. $\eta$ gives the marking on $\Omega_{s} / \eta\left(\pi_{1}\right)$ and hence determines the point of Teich $(\Sigma)$ and $\nu_{s}(\eta) \in \mathbb{H}$.

- $\Omega_{s}$ is an infinite union of disks and $\Omega_{s} / \eta\left(\pi_{1}\right)$ is a thrice punctured sphere obtained from the corresponding boundary of $\Sigma \times(0,1)$ by deleting a simple closed curve $\mathbf{w}(p / q)$ for some $p / q \in \hat{\mathbb{Q}}$. In this case, set $\nu_{s}(\eta)=p / q$.

- $\Omega_{s}$ is empty. In this case $\nu_{s}(\eta) \in \mathbb{R} \backslash \mathbb{Q}$. This is defined as the limit of rational numbers corresponding to simple closed curves exiting the end $e_{s}$.

The pair $\left(\nu_{-}(\eta), \nu_{+}(\eta)\right)$ is called the end invariants of $\eta$.

Fix $p / q \in \hat{\mathbb{Q}}$. Denote by $\lambda(\eta)=\lambda_{p / q}(\eta)$ the complex length of $\eta(\mathbf{w}(p / q))$ satisfying that $\operatorname{Re} \lambda(\eta) \geq 0$ and $-\pi<\operatorname{Im} \lambda(\eta) \leq \pi$. This satisfies the equation

$$
\operatorname{tr}^{2} \eta(\mathbf{a})=4 \cosh ^{2}(\lambda(\eta) / 2) .
$$

By Minsky's pivot theorem, Theorem 4.1 of [4], we have the following:

Proposition 3.1. There exist universal constants $\epsilon_{1}, c_{1}>0$ such that if $\operatorname{Re} \lambda(\eta)<$ $\epsilon_{1}$, then

$$
\frac{2 \pi i}{\lambda(\eta)} \approx_{c_{1}} T\left(\nu_{+}(\eta)\right)-T\left(\overline{\nu_{-}(\eta)}\right)+i
$$

where $T \in \mathrm{PSL}_{2}(\mathbb{Z})$ with $T(p / q)=\infty$.

Example 5. (F) Let $\eta$ be a fuchsian punctured torus group. Then $\nu_{+}(\eta)=$ $\nu_{-}(\eta) \in \mathbb{H}$.

(B) Let $G$ be a quasifuchsian group of type $(1,1)$. Then $\nu_{+}\left(\rho_{\varphi}^{b}\right)$ is the period on $\Omega_{\varphi}^{b} / \rho_{\varphi}^{b}\left(\pi_{1}\right)$ corresponding to the homology basis $([\mathbf{a}],[\mathbf{b}])$ on $\Sigma$ by the canonical homeomorphism induced from $\rho_{\varphi}^{b}$ and $\nu_{-}\left(\rho_{\varphi}^{b}\right) \equiv \nu_{0}$ for some $\nu_{0} \in \mathbb{H}$.

(E) $\nu_{+}\left(\rho_{d}^{e}\right)$ is the period on $\Omega_{d}^{e} / \rho_{d}^{e}\left(\pi_{1}\right)$ corresponding to the basis $([\mathbf{a}]$, [b] $)$ on $\Sigma$. $\nu_{-}\left(\rho_{d}^{e}\right)$ satisfies that $\overline{\nu_{+}\left(\rho_{d}^{e}\right)} \nu_{-}\left(\rho_{d}^{e}\right)=1$ since $\rho_{d}^{e}\left(\pi_{1}\right)$ admits an elliptic involution $z \rightarrow-z$ which conjugates $\rho_{d}^{e}(\mathbf{a})$ to $\rho_{d}^{e}(\mathbf{b})$ (cf. [29]).

(M) $\nu_{+}\left(\rho_{\mu}^{m}\right)$ is the period on $\Omega_{\mu}^{m} / \rho_{\mu}^{m}\left(\pi_{1}\right)$ corresponding to the basis $([\mathbf{a}],[\mathbf{b}])$ on $\Sigma$ and $\nu_{-}\left(\rho_{\mu}^{m}\right) \equiv 1 / 0$ for all $\mu \in M$.

\section{Trace functions and the Geometry of Parameter spaces}

4.1. Localization of holomorphic families. The following proposition is observed by Y. Komori and Y. Minsky (cf. [47] and [29]).

Proposition 4.1. All Bers slices, the Earle slice, and the Maskit slice are Jordan domains in their ambient spaces. Moreover, their canonical identifications with the Teichmüller space are extended homeomorphically to the Thurston compactification.

We easily deduce the following corollary from this proposition. 


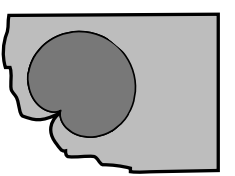

$\mathrm{n}=1$

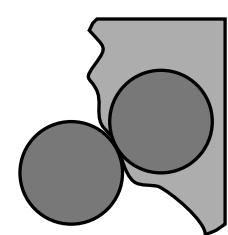

$\mathrm{n}=2$

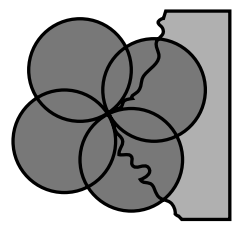

$\mathrm{n}>2$

FiguRE $5.2 \pi / n$-corners

Corollary 6. Let $\mathfrak{q} \in \mathbb{Q} \mathcal{X}$. Then $x(\mathfrak{q})$ admits a system of neighborhoods so that each element $U$ in this system satisfies the following:

(1) $\mathcal{X} \cap U$ is connected and contractible, and $U \cap \partial \mathcal{X}$ is a Jordan curve whose two end points are contained in $\partial U$.

(2) $U$ itself is connected and simply connected.

(3) Positive end invariant $\nu_{+}(x):=\nu_{+}\left(\left[\eta_{x}\right]\right)$ maps $U \cap \overline{\mathcal{X}}$ homeomorphically into $\overline{\mathbb{H}}$ so that $\nu_{+}(U \cap \mathcal{X}) \subset \mathbb{H}$ and $\nu_{+}(U \cap \partial \mathcal{X})$ is an open interval in $\hat{\mathbb{R}}=\partial \mathbb{H}$ containing $\mathfrak{q}$.

4.2. Geometry of parameter spaces. This subsection treats geometric properties of the parameter space for the holomorphic family of once punctured torus groups defined as in previous subsection.

4.2.1. $2 \pi / n$-corners. We define a $2 \pi / n$-corner and give its properties.

Definition $6\left(2 \pi / n\right.$-corner). Let $E$ be a proper subdomain in $\mathbb{C}$ and let $e_{0} \in \partial E \cap \mathbb{C}$. We say that $e_{0}$ is a $2 \pi / n$-corner of $E(n \in \mathbb{N})$ if one of the following conditions is satisfied (cf. Figure [5):

- If $n=1$, there exists a disk $D \subset \mathbb{C}$ such that $0 \in \partial D$ and $e_{0}+t^{2} \in E$ $(t \in D)$.

- If $n=2$, there exist two disjoint disks $D_{1}$ and $D_{2}$ such that $\overline{D_{1}} \cap \overline{D_{2}}=\left\{e_{0}\right\}$, $D_{1} \subset E$, and $D_{2} \cap E=\emptyset$.

- If $n \geq 3$, there are four disks $\left\{D_{j}, D_{j}^{\prime}\right\}_{j=1,2}$ such that $D_{j}$ and $D_{j}^{\prime}$ are tangent at $e_{0}(j=1,2), \partial D_{1}$ intersects $\partial D_{2}$ at $e_{0}$ in angle $2 \pi / n,\left(D_{1} \cup D_{2}\right) \cap E=\emptyset$, and $D_{1}^{\prime} \cap D_{2}^{\prime} \subset E$.

A $2 \pi$-corner is also called an inward-pointing cusp of $E$.

A $2 \pi / n$-corner of $E$ is a corner of angle $2 \pi / n$ for $E$ and is a corner of angle $2 \pi(1-1 / n)$ for the complementary domain $\operatorname{Int}(\complement E)$ of $E$. This similar notion is given in Pommerenke's book [54].

We note here two basic properties of $2 \pi / n$-corners. The first is used in the next subsection and the second in Section [10,

Lemma 4.1. Let $E$ be a proper subdomain in $\mathbb{C}$ and $e_{0} \in \partial E \cap \mathbb{C}$. Let $U$ be a neighborhood of $e_{0}$ and $\lambda$ a holomorphic function on $U$. Let $n$ be the order of zeros of the function $\lambda-\lambda\left(e_{0}\right)$ at $e_{0}$. Then $\lambda\left(e_{0}\right)$ is a $2 \pi / m$-corner of $\lambda\left(U_{0} \cap E\right)$ for some small neighborhood $U_{0}$ of $e_{0}$ if and only if $e_{0}$ is a $2 \pi /(n m)$-corner of $E$.

Proof. It can be easily deduced from the local properties of holomorphic functions (cf. [1]). 
Proposition 4.2. Let $\mathbb{B}$ be the unit disk in $\mathbb{C}$. There is no quasiconformal mapping $f$ such that $f(\hat{\mathbb{C}}-\overline{\mathbb{B}})$ admits an inward-pointing cusp. In particular, no quasiconformal mapping maps a $2 \pi / n$-corner $(n \geq 2)$ to an inward-pointing cusp.

Proof. Assume there was such a quasiconformal mapping $f$. We may assume that $f(1)=0$ and the origin is the inward pointing cusp. Let $\zeta_{1}, \zeta_{2} \in \partial f(\hat{\mathbb{C}}-\overline{\mathbb{B}})$ so that $\left|\zeta_{1}\right|=\left|\zeta_{2}\right|$. Since $f(\hat{\mathbb{C}}-\overline{\mathbb{B}})$ is a quasidisk, these points satisfy $\left|\zeta_{1}\right|<d\left|\zeta_{1}-\zeta_{2}\right|$ for some $d>0$ by Ahlfors' three points property (see [17]). On the other hand, since the origin is an inward pointing cusp, $\left|\zeta_{1}-\zeta_{2}\right|=O\left(\left|\zeta_{1}\right|^{2}\right)$. This contradicts the previous inequality. The last claim is proved by using a quasiconformal mapping which maps the sector of angle $2 \pi / n$ to the sector of angle $\pi$.

Corollary 7. No quasi-disk admits inward-pointing cusps.

4.2.2. Cusps in parameter space. In this section, we give a necessary and sufficient condition (Proposition 4.3) for a boundary point of the parameter space to be a $(2,3)$-cusp. To do this, we begin with the following lemma.

Lemma 4.2. Let $E$ be a domain with the following property: There exists a neighborhood $U$ of zero and two positive numbers $r_{1}>r_{2}$ such that

$$
\left\{\zeta \in \mathbb{C}|| \zeta-r_{2} \mid<r_{2}\right\} \subset E \cap U \subset\left\{\zeta \in \mathbb{C}|| \zeta-r_{1} \mid<r_{1}\right\} .
$$

Let $\xi=h(\zeta)$ be an odd univalent function on $U$. Then the origin is a $(2,3)$-cusp of the domain $E^{\prime}:=\left\{h(\zeta)^{2} \mid \zeta \in E \cap U\right\}$.

Proof. Replacing $E$ with $E \cap U$ if necessary, we may assume that $E \subset U$. By Lemma 4.1, the origin is an inward-pointing cusp of $E^{\prime}$. Hence there exist $a, r>0$ and $\theta \in \mathbb{R}$ such that $e^{i \theta} \cdot C^{+}(a, r) \subset E^{\prime}$. Therefore, to complete this lemma, we should find a positive number $b$ such that $e^{i \theta} \cdot C^{-}(b, r) \cap \overline{E^{\prime}}=\emptyset$ with suitable $r>0$. Especially, since $E^{\prime}$ contains a cardioid whose cusp is the origin, it suffices to show the following weaker condition: there exist $\theta^{\prime} \in \mathbb{R}$ and $b>0$ such that $e^{\theta^{\prime}} \cdot C^{-}(b, r) \cap \overline{E^{\prime}}=\emptyset$. Indeed, if such $\theta^{\prime}$ exists, $\theta^{\prime}$ is automatically equal to $\theta$ modulo $2 \pi$.

We may assume that $h^{\prime}(0)=1$. Since $h$ is an odd univalent function, we have

$$
h(-\zeta)=-h(\zeta) \text { and }|h(\zeta)-\zeta|=O\left(|\zeta|^{3}\right) .
$$

By definition, $E \cap(-E)=\emptyset$ and $E_{0}:=E \cup(-E)$ is contained in the union of two circles $\left\{\zeta|| \zeta \pm r_{1} \mid<r_{1}\right\}$. Therefore, the equations in (1) imply that $h(E) \cap(-h(E))=$ $\emptyset, h\left(E_{0}\right)=h(E) \cup(-h(E))$ and there exist $r_{1}^{\prime}>0$ and a neighborhood $V$ of $w=0$ so that

$$
h\left(E_{0}\right) \cap V \subset\left\{\zeta \in \mathbb{C} \| \zeta+r_{1}^{\prime} \mid<r_{1}^{\prime}\right\} \cup\left\{\zeta \in \mathbb{C} \| \zeta-r_{1}^{\prime} \mid<r_{1}^{\prime}\right\} .
$$

Hence $h\left(E_{0}\right) \cap V$ does not intersect a union of two cones $C^{\prime}=\left\{\zeta \| \zeta \pm r_{1}^{\prime} \mid>r_{1}^{\prime}\right.$, $\left.|\zeta|<r_{2}^{\prime}\right\}$, some small $r_{2}^{\prime}>0$. Thus, the set $\left\{\zeta^{2} \mid \zeta \in C^{\prime}\right\}$ contains $(-1) \cdot C^{-}\left(b,\left(r_{2}^{\prime}\right)^{2}\right)$ for suitable $b>0$, which implies $E \cap(-1) \cdot C^{-}(b, r)=\emptyset$ for some $r>0$.

The following proposition is a refinement of the main result of the author's previous paper 48 .

Proposition 4.3. Let $\mathfrak{q} \in \mathbb{Q} \mathcal{X}$. If $x(\mathfrak{q})$ is a zero of the function $\operatorname{tr}^{2} \eta_{x}(\mathbf{w}(\mathfrak{q}))-4$ of order $n$, then $x(\mathfrak{q})$ is a $2 \pi / n$-corner of $\mathcal{X}$. Furthermore, the derivative of the trace function at $x(\mathfrak{q})$ does not vanish if and only if $x(\mathfrak{q})$ is a $(2,3)$-cusp of $\mathcal{X}$. 
Proof. Let $\Pi(t)=x(\mathfrak{q})+t^{2}$ and let $\tilde{\mathcal{X}}$ be a lift of $\mathcal{X}$ via the covering mapping $\Pi$. To prove the first part of this proposition, it suffices to show from Lemma 4.1 that the origin $t=0$ is a $\pi / n$-corner of $\tilde{\mathcal{X}}$.

Let $\lambda_{\mathfrak{q}}$ denote the complex length of $\eta_{\Pi(t)}(\mathbf{w}(\mathfrak{q}))$ for $t \in \tilde{\mathcal{X}}$. By definition,

$$
\operatorname{tr}^{2} \eta_{\Pi(t)}(\mathbf{w}(\mathfrak{q}))-4=4 \sinh ^{2} \frac{\lambda_{\mathfrak{q}}(t)}{2} .
$$

Put $\tilde{\mathcal{X}}_{0}=\Pi^{-1}\left(\mathcal{X}_{0}\right) \subset \mathbb{C}$. We now have two branched covering spaces $\Pi: \tilde{\mathcal{X}}_{0} \rightarrow \mathcal{X}_{0}$ and $\sinh ^{2}(-): \mathbb{C} \rightarrow \mathbb{C}$. Note that these two projections $\Pi$ and $\sinh ^{2}(-)$ are branched of order 2 at $x(\mathfrak{q})$ and the origin, respectively. Therefore, taking the lift of the map $\mathcal{X}_{0} \ni x \mapsto \operatorname{tr}^{2} \eta_{x}-4 \in \mathbb{C}$, we may assume that $\lambda_{\mathfrak{q}}$ is holomorphic on a neighborhood $V$ of the origin $t=0$ and satisfies that $\operatorname{Re} \lambda_{\mathfrak{q}}>0$ in $\tilde{\mathcal{X}}$ and $\lambda_{\mathfrak{q}}(0)=0$. Since the holomorphic mapping $\lambda_{\mathfrak{q}}$ is a lift of two covering spaces as above, the mapping commutes with their covering transformations $t \mapsto-t$ of $\Pi$ and $z \rightarrow-z$ of $\sinh ^{2}(-)$. This means that $\lambda_{\mathfrak{q}}$ is an odd function on $V$. By assumption, $x(\mathfrak{q})$ is a zero of the left-hand side of (2) of order $n$. Therefore the origin is a zero of $\lambda_{\mathfrak{q}}$ of order $n$. Shrinking $V$ if necessary, we may suppose that the origin is the unique zero of $\lambda_{\mathfrak{q}}$ in $V$ and

$$
\left|\operatorname{Re} \lambda_{\mathfrak{q}}(t)\right|<\epsilon_{1}, \quad\left|\operatorname{Im} \lambda_{\mathfrak{q}}(t)\right|<\pi, t \in V,
$$

where $\epsilon_{1}$ is the positive constant given in Minsky's pivot theorem (Proposition 3.1). We may also assume $V$ to be invariant under the covering transformation of $\Pi: \tilde{\mathcal{X}}_{0} \rightarrow \mathcal{X}_{0}$ and such that $U:=\Pi(V)$ becomes a neighborhood of $x(\mathfrak{q})$ satisfying the conditions (1), (2), and (3) in Corollary 6 ,

Put $\nu_{ \pm}(x)=\nu_{ \pm}\left(\left[\eta_{x}\right]\right)$ for $x \in \mathcal{X}$. Then by (3), we can apply Proposition 3.1 for ends invariants of $\eta_{\Pi(t)}$ for all $t \in \overline{\mathcal{X}} \cap V$, and hence we obtain

$$
\frac{2 \pi i}{\lambda_{\mathfrak{q}}(t)} \approx_{c_{1}} T\left(\nu_{+}(\Pi(t))\right)-\overline{T\left(\nu_{-}(\Pi(t))\right)}+i, \quad t \in \overline{\tilde{\mathcal{X}}} \cap V,
$$

where $T \in \mathrm{PSL}_{2}(\mathbb{Z})$ with $T(\mathfrak{q})=\infty$.

In the cases of a Bers slice and the Maskit slice, the end invariant for the negative end is a suitable fixed point in $\overline{\mathbb{H}}$. In the Earle slice case $\mathcal{X}=\mathcal{E}$, the end invariant for the negative end of $\left[\eta_{x(\mathfrak{q})}\right]$ is $\mathfrak{q}^{-1}$. Since $\overline{\nu_{+}(x)} \nu_{-}(x)=1$ for $x \in \mathcal{E}$ and $\nu_{+}$is the Riemann mapping of $\mathcal{E}, \nu_{-}$is continuous on the closure of $\mathcal{E}$. Hence, shrinking $V$ again if necessary, we may suppose

$$
\left|\operatorname{Re} T\left(\nu_{-}(x)\right)-T\left(\mathfrak{q}^{-1}\right)\right|<1 \quad \text { and } \quad 0<\operatorname{Im} T\left(\nu_{-}(x)\right)<1, \quad x \in U \cap \mathcal{E} .
$$

Let $I_{0}$ be an interval in $\mathbb{R}$ corresponding to the curve $\nu_{+}(U) \cap \overline{\mathcal{X}}$ via $\nu_{+}$. Then the mapping $I_{0} \ni \Theta \mapsto x(\Theta) \in U$ is well-defined and satisfies $\nu_{+}(x(\Theta))=\Theta$. Take a lift $\tilde{x}(-)$ from $I$ to $\partial \tilde{\mathcal{X}} \cap V$ so that $\Pi \circ \tilde{x}(\Theta)=x(\Theta)$. Then by (4) and from the observation on the negative end invariant, there exists a positive constant $c_{2}$ and $\nu_{0} \in \overline{\mathbb{H}}$ so that

$$
\frac{2 \pi i}{\lambda_{\mathfrak{q}}((\tilde{x}(\Theta))} \approx_{c_{2}} T(\Theta)-\overline{\nu_{0}}+i, \quad \Theta \in I_{0}
$$

This implies that

$$
\left\{\lambda \in \mathbb{C}|| \lambda-r_{1} \mid<r_{1}\right\} \subset \lambda_{\mathfrak{q}}(\tilde{\mathcal{X}} \cap V) \subset\left\{\lambda \in \mathbb{C}|| \lambda-r_{2} \mid<r_{2}\right\}
$$

for some $r_{1}, r_{2}>0$. Thus $\lambda=0$ is a $\pi$-corner of a domain $\lambda_{\mathfrak{q}}(V \cap \tilde{\mathcal{X}})$ and hence, the origin $t=0$ is a $\pi / n$-corner of $\tilde{\mathcal{X}}$ by Corollary 6 
Next we show the rest of this proposition. If $x(\mathfrak{q})$ is a $(2,3)$-cusp of $\mathcal{X}$, the derivative of the trace function at $x(\mathfrak{q})$ does not vanish by above observation. The converse follows from (5) and Lemma 4.2

\section{Pleating Rays}

Details of the contents of this subsection can be found in [15], 24], 30, and [44]. Recall that for any point $x \in \mathcal{X}$, a Kleinian group $G_{x}$ admits the distinguished invariant component $\Omega_{x}$, which is one of the three; $\Omega_{\varphi}^{b}, \Omega_{d}^{e}$, or $\Omega_{\mu}^{m}$.

Let $p / q \in \mathbb{Q} \mathcal{X}$. The $p / q$-pleating ray $\mathcal{P}_{p / q}^{\mathcal{X}}$ is the set of points $x \in \mathcal{X}$ so that the component of the boundary of the convex core of $\mathbb{H}^{3} / G_{x}$ facing $\Omega_{x} / G_{x}$ is bent along the simple closed geodesic labeled by $p / q$. In all cases, the $p / q$-pleating ray is an embedded real analytic curve on $\mathcal{X}$ landing at $x(p / q)$, and $X_{p / q, x}$ remains a hyperbolic element (i.e. without screw actions along its axis) on the ray.

Remark 1 . This paper treats the pleating rays only in the rational case; that is, the rays corresponding to rational laminations (see [26] for more general situations).

Proposition 5.1. The translation length of $X_{p / q, x}$ on $\mathcal{P}_{p / q}^{\mathcal{X}}$ gives a real analytic diffeomorphism from $\mathcal{P}_{p / q}^{\mathcal{X}}$ onto its image. Further, from the inside of $\mathcal{P}_{p / q}^{\mathcal{X}}, x$ tends to $x(p / q)$ if and only if the translation length tends to zero.

Here, for a hyperbolic transformation $W$, the translation length of $W$ is the positive real number $l$ satisfying that $\operatorname{tr}^{2} W=4 \cosh ^{2}(l / 2)$. To prove this proposition, it suffices to show that the derivative of the trace function $\operatorname{tr}^{2} X_{p / q, x}$ does not vanish along $\mathcal{P}_{p / q}^{\mathcal{X}}$. Indeed, in the cases $\mathcal{X}=\mathcal{B}_{G}$ or $\mathcal{E}$, this can be proved by combining the Local Pleating Theorem due to L. Keen and C. Series (cf. [26] see also Section[12) and the fact that all pleating rays are embedded arcs (see also Theorem 5.1 of 30 . and Theorem 7.4 of [44]). Notice that the case of $\mathcal{X}=\mathcal{M}$ had already been proven directly in Lemma 5.5 of [24].

\section{Geometry on the Boundaries of CONVEX CORES}

In this and the next sections, we treat any (but not fuchsian) once punctured torus groups with distinguished invariant component.

This section deals with basic properties of $F$-peripheral subgroups and peripheral disks. Many results of this section seem to be well-known due to L. Keen and C. Series' beautiful works (cf. [24], 25], and [26]). For the convenience of the readers, we give a brief proof of them here. Let $\eta$ be a once punctured torus group with distinguished invariant component $\Omega$. To simplify the argument, we assume throughout this section that $G:=\eta\left(\pi_{1}\right)$ is not a fuchsian group.

6.1. The expanding mapping. Denote by $\mathcal{C}_{\eta}$ the convex hull of the limit set of $G$ and by $\partial_{0} \mathcal{C}_{\eta}$ the component of $\partial \mathcal{C}_{\eta}$ facing $\Omega$. Then $\partial_{0} \mathcal{C}_{\eta}$ is simply connected and has a hyperbolic structure inherited from $\mathbb{H}^{3}$. Therefore, there exists a hyperbolic structure on $\Sigma$ and an isometry from the universal covering of $\Sigma$ to $\partial_{0} \mathcal{C}_{\eta}$. By the expanding mapping, we here mean this isometry. The expanding mapping induces an isomorphism between $\pi_{1}$ to $G$ compatible with $\eta$. 


\subsection{F-peripheral subgroups and peripheral disks.}

Definition 7 ( $F$-peripheral subgroup). Let $G$ be a Kleinian group. A fuchsian subgroup $H$ of $G$ is called $F$-peripheral if the convex hull of the limit set of $H$ is contained in the boundary of the convex hull of the limit set of $G$. Let $\Omega$ be a component of $G$. If the convex hull of $\Lambda(H)$ is contained in the boundary of the convex hull of $\Lambda(G)$ facing $\Omega$, the subgroup $H$ is also said to be $F$-peripheral with respect to $\Omega$.

Lemma 6.1. Let $\eta, G, \Omega$, and $\partial_{0} \mathcal{C}_{\eta}$ be as in the previous subsection. Suppose $\partial_{0} \mathcal{C}_{\eta}$ is bent along the geodesic corresponding to $\eta(\mathbf{w}(p / q))$ for some $p / q \in \hat{\mathbb{Q}}$. Let $X \in G$ be an element conjugate to $\eta(\mathbf{w}(p / q))$ in $G$ and $V \in G$ such that $G=\langle X, V\rangle$. Then a subgroup $H=\left\langle X, V X V^{-1}\right\rangle$ is $F$-peripheral with respect to $\Omega$

To show Lemma 6.1, we shall prove the following proposition.

Proposition 6.1. Let $H$ be as in Lemma 6.1, Then, there exists the unique disk $\Delta(H)$ such that $\Delta(H) \subset \Omega$ and $\Lambda(H)=\overline{\Delta(H)} \cap \Lambda(G)$.

Proof. According to Proposition A.1 of 24], $H$ is a fuchsian group with invariant circle $C$ such that the quotient space of each component of $\hat{\mathbb{C}}-C$ by $H$ is a once punctured cylinder whose boundary curves are represented by $X$ and $V X V^{-1}$. Especially, $\Lambda(H) \subset C$. Since $\eta^{-1}(H)$ is conjugate to the fundamental group of $\Sigma-\mathbf{w}(p / q)$ in $\pi_{1}$ and the axis of $\eta(\mathbf{w}(p / q))$ is a bending line, by passing through the expanding mapping of $\partial_{0} \mathcal{C}_{\eta}$, we can see that $H$ stabilizes some flat piece $N_{0}$ of $\partial_{0} \mathcal{C}_{\eta}$. This implies that $\Lambda(H) \subset N_{0} \cap \hat{\mathbb{C}}$. Since $\Lambda(H)$ contains at least 3 points, the totally geodesic plane in $\mathbb{H}^{3}$ containing $N_{0}$ coincides with the plane with contour $C$. Since $\partial_{0} \mathcal{C}_{\eta}$ is a component of the boundary of the convex set $\mathcal{C}_{\eta}$, there exists the unique component $\Delta(H)$ of $\hat{\mathbb{C}}-C$ so that the totally geodesic plane whose boundary is $C$ becomes the support plane which separates $\Delta(H)$ and $\mathcal{C}_{\eta}$. Because $\partial_{0} \mathcal{C}_{\eta}$ faces $\Omega$, the disk $\Delta(H)$ is contained in $\Omega$.

Next, we shall show that $\Lambda(H)=\overline{\Delta(H)} \cap \Lambda(G)$. Immediately, $\Lambda(H) \subset \overline{\Delta(H)} \cap$ $\Lambda(G)$. Let $H^{\prime}=\left\langle X, V^{-1} X V\right\rangle$. Then, $H^{\prime}$ is also an $F$-peripheral subgroup contain$\operatorname{ing} X$ and satisfies $\Delta\left(H^{\prime}\right) \neq \Delta(H)$. Indeed, assume that $\Delta\left(H^{\prime}\right)=\Delta(H)$. Since $V H^{\prime} V^{-1}=H, V(\Delta(H))=V\left(\Delta\left(H^{\prime}\right)\right)=\Delta(H)$. Thus $G=\langle H, V\rangle$ stabilizes a disk $\Delta(H)$. This is a contradiction because it means $G$ is fuchsian.

Let $\sigma$ be the component of $\partial \Delta(H)-\Lambda(H)$ which connects the fixed points of $X$. Since both $\partial \Delta(H)$ and $\partial \Delta\left(H^{\prime}\right)$ contain the fixed points of $X, \partial \Delta(H) \cap \partial \Delta\left(H^{\prime}\right)$ consists of the fixed points of $X$. Then we have $\sigma \subset \Delta\left(H^{\prime}\right) \subset \Omega$ because $\partial \Delta(H)-\bar{\sigma}$ contains a limit point of $H$. By the same reason, the component $\sigma^{\prime}$ of $\partial \Delta(H)-\Lambda(H)$ connecting the fixed points of $V X V^{-1}$ is included in $\Omega$.

By applying Theorem A.1 of [24] again, we have $\partial \Delta(H)-\Lambda(H)=\bigcup_{h \in H} h(\sigma) \cup$ $h\left(\sigma^{\prime}\right)$. Thus we conclude the assertion.

Proof of Lemma 6.1] Since $\Delta(H) \subset \Omega$ and $\Delta(H) \cap \Lambda(G)=\Lambda(H)$, the convex hull of $\Lambda(H)$ is contained in $\partial_{0} \mathcal{C}_{\eta}$.

Almost all $F$-peripheral subgroups treated in this paper form as in Lemma 6.1 For simplicity of notation, we define

Definition 8 (Peripheral subgroups and disks). Let $G$ be a once punctured torus group with invariant component. Assume that a component of the boundary of 
the convex core of the manifold $\mathbb{H}^{3} / G$ is bent along the simple closed geodesic. A subgroup $H$ of $G$ is called a peripheral subgroup if there exists $X, V \in G$ such that $X$ is a primitive and hyperbolic element whose axis corresponds to a bending locus, $G=\langle X, V\rangle$, and $H=\left\langle X, V^{-1} X V\right\rangle$. The disk $\Delta(H)$ as shown in Proposition 6.1 is called the peripheral disk of $H$.

Proposition 6.2. $\quad$ (1) Let $H$ be a peripheral subgroup of $G$. Let $A$ be a Möbius transformation. Then $A H A^{-1}$ is a peripheral subgroup of $A G A^{-1}$ with a peripheral disk $A(\Delta(H))$.

(2) The stabilizer subgroup of a peripheral disk is a peripheral subgroup.

(3) Let $X$ be an element in $G$ whose axis is a bending locus. Then there are exactly two peripheral subgroups containing $X: H_{1}=\left\langle X, V^{-1} X V\right\rangle$ and $H_{2}=\left\langle X, V X V^{-1}\right\rangle$, where $V \in G$ with $G=\langle X, V\rangle$.

Proof. (1) By the definition.

(2) This follows from L. Keen and C. Series' argument in Lemma 3.4 of [25].

(3) From the proof of Proposition 6.1, $\Delta\left(H_{1}\right) \neq \Delta\left(H_{2}\right)$. Hence $\partial \Delta\left(H_{1}\right) \cap$ $\partial \Delta\left(H_{2}\right)$ consists of the fixed points of $X$. Let $H$ be an $F$-peripheral subgroup containing $X$. Then $\partial \Delta(H)$ passes through the fixed points of $X$. Since $\Lambda(H) \subset$ $\partial \Delta(H)$ and $\Delta(H) \subset \Omega, \Delta(H)$ must coincide with one of $\left\{\Delta\left(H_{i}\right)\right\}_{i=1,2}$. From (2), $H$ should agree with one of those peripheral subgroups.

Proposition 6.3. Let $H$ be a peripheral subgroup of $G$. Let $W \in G$. Then the set $\operatorname{Fix}(W)$ of fixed points of $W$ satisfies either $\operatorname{Fix}(W) \subset \Lambda(H)$ or $\operatorname{Fix}(W) \cap \overline{\Delta(H)}=\emptyset$. Furthermore, $\operatorname{Fix}(W) \subset \Lambda(H)$ if and only if $W \in H$.

To prove this, we use the following theorem by Susskind (Theorem[2 of [59]).

Theorem (Susskind). Let $G$ be a Kleinian group containing no rank-2 parabolic subgroups. Let $\mathrm{H}_{1}$ and $\mathrm{H}_{2}$ be a geometrically finite subgroup of $G$. Then $\Lambda\left(H_{1} \cap H_{2}\right)=\Lambda\left(H_{1}\right) \cap \Lambda\left(H_{2}\right)$.

Proof of Proposition 6.3. Assume that $\langle W\rangle \cap H=\emptyset$. Since $G$ contains no rank-2 parabolic subgroup and $H$ and $\langle W\rangle$ are geometrically finite subgroups of $G$,

$$
\Lambda(H) \cap \operatorname{Fix}(W)=\Lambda(H) \cap \Lambda(\langle W\rangle)=\Lambda(H \cap\langle W\rangle)=\emptyset .
$$

Clearly, $\operatorname{Fix}(W) \subset \Lambda(H)$ when $\langle W\rangle \cap H \neq \emptyset$. Thus we conclude the first statement.

Now, we assume that $\operatorname{Fix}(W) \subset \Lambda(H)(\subset \partial \Delta(H))$. By (2) of Proposition 6.2 we have only to show $W(\Delta(H))=\Delta(H)$.

If $W$ is parabolic, one of disks $\{W(\Delta(H)), \Delta(H)\}$ is contained within the other. Since a part of $\Lambda(H)$ lies on their disks, both disks must coincide.

Assume $W$ is loxodromic. If $W^{2}(\Delta(H))=\Delta(H)$ but $W(\Delta(H)) \neq \Delta(H)$, then $W(\Delta(H)) \cap \Delta(H)=\emptyset$ and $\partial W(\Delta(H))=\partial \Delta(H)$. Since $\Delta(H)$ is contained in the invariant component $\Omega, \Lambda(G) \subset \partial \Delta(H)$. This means $G$ is fuchsian and contradicts our assumption. Finally, suppose that that $W(\Delta(H)), W^{2}(\Delta(H)) \neq \Delta(H)$. In this case, we can see that $\bigcup_{n \in \mathbb{Z}} W^{n}(\Delta(H))=\hat{\mathbb{C}}-\operatorname{Fix}(W)$. This implies that $\Lambda(G) \subset \operatorname{Fix}(W)$ because $\Delta(H) \subset \Omega$. Since $G$ is non-elementary, this case cannot occur.

Remark 2. The author learned of Susskind's result from Professor Caroline Series. He would like to thank for her accurate advice. 
Proposition 6.4. Let $H_{1}$ and $H_{2}$ be peripheral subgroups of $G$ with respect to the distinguished invariant component $\Omega$. If there is an element $X \in G$ so that $X$ is conjugate to $\eta(\mathbf{w}(p / q))$ and $\partial \Delta\left(H_{1}\right) \cap \partial \Delta\left(H_{2}\right)=\operatorname{Fix}(X)$, then $H_{1} \cap H_{2}=\langle X\rangle$.

Proof. Clearly $H_{1} \neq H_{2}$ because $\Delta\left(H_{1}\right) \neq \Delta\left(H_{2}\right)$. Since $\operatorname{Fix}(X) \subset \partial \Delta\left(H_{i}\right)(i=1,2)$ and $\operatorname{Fix}(X) \subset \Lambda(G), \operatorname{Fix}(X) \subset \Lambda(H)$. Hence $X$ is contained in $H_{i}(i=1,2)$ from Proposition 6.3. Since each $H_{i}$ corresponds to the fundamental group of $\Sigma-\mathbf{w}(p / q)$ and $X$ is conjugate to $\eta(\mathbf{w}(p / q)), H_{1} \cap H_{2}$ must agree with $\langle X\rangle$.

6.3. Intersections among peripheral disks. Let $\eta, X$, and $V$ be as above. From observations in the previous subsection, the axes of $X$ and $V^{-1} X V$ both lie on $\partial_{0} \mathcal{C}_{\eta}$ and they bound a piece of hyperbolic plane $P$. The bending angle is the angle in the interval $[0, \pi)$ between $P$ and $V(P)$, so that it is zero when $P$ and $V(P)$ are coplanar, and is near $\pi$ when $P$ and $V(P)$ are almost parallel in $\mathbb{H}^{3}$, which corresponds to the case where $X$ is close to parabolic.

Proposition 6.5. Let $\eta$ be a once punctured torus group with distinguished invariant component $\Omega$. Then for any $\epsilon_{2}$ with $0<\epsilon_{2}<\pi$, there exists a constant $\kappa_{0}=\kappa_{0}\left(\epsilon_{2}\right)>0$ which satisfies the following: Let $p / q \in \hat{\mathbb{Q}}$. Suppose that

(1) $\partial_{0} \mathcal{C}_{\eta}$ is bent along the axis of $\eta(\mathbf{w}(p / q))$,

(2) its bending angle is more than $\epsilon_{2}$, and

(3) the translation length of $\eta(\mathbf{w}(p / q))$ is less than $\kappa_{0}$.

Let $H$ be a peripheral subgroup of $G:=\eta\left(\pi_{1}\right)$ and $W \in G-H$. Then $W(\Delta(H)) \cap$ $\Delta(H) \neq \emptyset$ if and only if $\partial W(\Delta(H)) \cap \partial \Delta(H)$ consists of the fixed points of an element of $G$ conjugate to $\eta(\mathbf{w}(p / q))$.

It is easy to see the "if"-part of this proposition. To show the "only if" part, we begin with the following lemma.

Lemma 6.2. Under the hypothesis of Proposition 6.5, for any $\epsilon_{2}$ with $0<\epsilon_{2}<\pi$, there exists a constant $\kappa_{0}=\kappa_{0}\left(\epsilon_{2}\right)>0$ satisfying the following: Let $H$ and $H^{\prime}$ be peripheral subgroups of $G$ with $H \cap H^{\prime}=\langle X\rangle$ where $X$ is conjugate to $\eta(\mathbf{w}(p / q))$. Suppose that the bending angle $\theta^{\prime}$ is more than $\epsilon_{0}$. Then, if the translation length $l$ of $\eta(\mathbf{w}(p / q))$ is less than $\kappa_{0}, \Delta(H) \cap \Delta\left(H^{\prime}\right)$ is contained in a component of the preimage of the Margulis collar of the geodesic in $\Omega / G$ which is homotopic to $\mathbf{w}(p / q)$.

Proof. By taking conjugation if necessary, we may suppose that $X(\zeta)=e^{l} \zeta$ for some $l>0$ and $\Delta(H) \cap \Delta\left(H^{\prime}\right)$ is the sector $\left\{\zeta \in \mathbb{C}|| \arg \zeta \mid<\left(\pi-\theta^{\prime}\right) / 2\right\}$. Then, the invariant component $\Omega$ contains the sector

$$
\Delta(H) \cup \Delta\left(H^{\prime}\right)=\left\{\zeta \in \mathbb{C}|| \arg \zeta \mid<\left(\pi+\theta^{\prime}\right) / 2\right\} .
$$

Hence by a straightforward calculation, we have that

$$
d_{\Omega}(\zeta, X(\zeta)) \leq d_{\Delta(H) \cup \Delta\left(H^{\prime}\right)}(\zeta, X(\zeta)) \leq \frac{2 l}{\left(\pi+\theta^{\prime}\right) \cos \left\{\pi\left(\pi-\theta^{\prime}\right) / 2\left(\pi+\theta^{\prime}\right)\right\}} \lesssim l
$$

for all $\zeta \in \Delta(H) \cap \Delta\left(H^{\prime}\right)$, where the implicit constant in the inequality is dependent only on $\epsilon_{2}$. Therefore there exists a constant $\kappa_{0}=\kappa_{0}\left(\epsilon_{2}\right)>0$ so that if $l<$ $\kappa_{0}$, the distance $d_{\Omega}(\zeta, X(\zeta))$ is less than the Margulis constant, which implies the assertion. 
Proof of Proposition 6.5. Take a positive constant $\kappa_{0}$ as in Lemma 6.2 Assume that the translation length of $\eta(\mathbf{w}(p / q))$ is less than $\kappa_{0}$.

Let $W \in G-H$. We suppose that $W(\Delta(H)) \cap \Delta(H) \neq \emptyset$. Notice that $W(\Delta(H))$ is the peripheral disk of $H^{\prime}=W H W^{-1}$ and $W(\Delta(H)) \neq \Delta(H)$ by (2) of Proposition 6.2 We take $X, X^{\prime} \in G$ with the properties that both are conjugate to $\eta(\mathbf{w}(p / q))$, and $\partial \Delta(H) \cap \Delta\left(H^{\prime}\right)$ (resp. $\left.\partial \Delta\left(H^{\prime}\right) \cap \Delta(H)\right)$ is contained in the free side of $H$ (resp. $\left.H^{\prime}\right)$ connecting $\operatorname{Fix}(X)\left(\right.$ resp. Fix $\left.\left(X^{\prime}\right)\right)$. Take peripheral subgroups $H_{1}$ and $H_{1}^{\prime}$ so that $H \cap H_{1}=\langle X\rangle$ and $H^{\prime} \cap H_{1}^{\prime}=\left\langle X^{\prime}\right\rangle$. Then both $\Delta(H) \cap \Delta\left(H_{1}\right)$ and $\Delta\left(H^{\prime}\right) \cap \Delta\left(H_{1}^{\prime}\right)$ contain $\Delta(H) \cap \Delta\left(H^{\prime}\right)$, and hence both sets do intersect. Since both of these sets are contained in the preimage of the Margulis collar with respect to $\mathbf{w}(p / q)$ in $\Omega / G$ by Lemma 6.2, applying Keen-Halpern's collar lemma (cf. e.g. [1]), we have $\langle X\rangle=\left\langle X^{\prime}\right\rangle$. This concludes the assertion.

6.4. Radius of peripheral disks. At the end of this section, we shall give the following proposition which is used in Section 10 to prove Theorem 12 .

Proposition 6.6 (Radius of peripheral disks). Let $\eta$ be a once punctured torus group with distinguished invariant component $\Omega$. Assume that $\eta$ satisfies the conditions (1), (2), and (3) in Proposition 6.5 for some $\epsilon_{2}$ and $\kappa_{0}>0$. Assume furthermore that there exists a loxodromic element $W \in G$ which forms $W(\zeta)=e^{\lambda} \zeta$ with $\operatorname{Re} \lambda>0$ and does not belong to any peripheral subgroup of $G$. Then for any $r>0$, there exists $\delta_{0}$ depending only on $\operatorname{Re} \lambda$ and $r$ such that the radius of a peripheral disk which intersects a disk $\{|\zeta|<r\}$ is less than $\delta_{0}$.

To prove this proposition, we begin with two lemmas; the first one comes from an elementary of the Number theory and the second from Euclidean geometry.

Lemma 6.3. There exists a universal constant $\mathfrak{a}_{0}>0$ satisfying the following: For any $b \in \mathbb{R}$, there are integers $a$ and $c$ such that $2 \leq a \leq \mathfrak{a}_{0}$ and $|a b-2 c|<1 / 3$.

Proof. For $c / a \in \mathbb{Q}$, we consider the following neighborhood

$$
\mathcal{N}_{c / a}=\{b \in \mathbb{R}|| b-(c / a) \mid<1 /(6 a)\} .
$$

Then the collection $\left\{\mathcal{N}_{c / a}\right\}_{c / a \in \mathbb{Q}}$ forms a covering of $\mathbb{R}$. Indeed, it is clear that all rational numbers are contained in the union of this covering system. By using the best approximations for irrational numbers (see e.g. 19]), for an irrational number $b$, we can find $c / a \in \mathbb{Q}$, such that $a \geq 6$ and $|b-(c / a)|<1 / a^{2} \leq 1 / 6 a$. This means that $b \in \mathcal{N}_{c / a}$ for some $c / a \in \mathbb{Q}$.

Since the interval $[0,1]$ is compact, we can find a finite subcollection $\left\{\mathcal{N}_{c_{i} / a_{i}}\right\}_{i}$ which covers the interval. Put $\mathfrak{a}_{0}=2 \times \max _{i}\left\{a_{i}\right\}$.

For any $b \in \mathbb{R}$, there exists $m \in \mathbb{Z}$ such that $b-m \in[0,1]$. By the argument above, $b-m \in \mathcal{N}_{c_{i} / a_{i}}$ for some $i$. Therefore, $a=2 a_{i}$ and $c=c_{i}+m \cdot a_{i}$ are desired numbers for given $b$.

Lemma 6.4. Let $\left.\Delta=\left\{\mid \zeta-\zeta_{0}\right\}<\delta\right\}$ be a disk in $\mathbb{C}$. Assume that $\Delta \cap\{|\zeta|<r\} \neq \emptyset$ for some $r>0$. Then for a complex number $\lambda$ with $\operatorname{Re} \lambda>0$ and $|\operatorname{Im} \lambda-2 \pi c|<\pi / 3$ for some $c \in \mathbb{Z}$, there exists $\delta_{0}^{\prime}$ depending only on $r$ and $\operatorname{Re} \lambda$ such that $\delta \geq \delta_{0}^{\prime}$ implies $e^{\lambda} \cdot \Delta \cap \Delta \neq \emptyset$.

Proof. The constant $\delta_{0}^{\prime}$ which is defined directly by

$$
\delta_{0}^{\prime}:=\frac{r\left(1+e^{\operatorname{Re} \lambda}+e^{2 \operatorname{Re} \lambda}\right)^{1 / 2}}{1+e^{\operatorname{Re} \lambda}-\left(1+e^{\operatorname{Re} \lambda}+e^{2 \operatorname{Re} \lambda}\right)^{1 / 2}}
$$


satisfies the assertion. Indeed by definition, the center and the radius of $e^{\lambda} \cdot \Delta$ are $e^{\lambda} \zeta_{0}$ and $e^{\operatorname{Re} \lambda} \delta$, respectively. Moreover $\left|\zeta_{0}\right|<r+\delta$ since $\Delta \cap\{|\zeta|<r\} \neq \emptyset$. Thus the assumption that $|\operatorname{Im} \lambda-2 \pi c|<\pi / 3$ for some $c \in \mathbb{Z}$ implies

$$
\begin{aligned}
\left|e^{\lambda} \zeta_{0}-\zeta_{0}\right| & \leq\left(1+e^{\operatorname{Re} \lambda}+e^{2 \operatorname{Re} \lambda}\right)^{1 / 2}\left|\zeta_{0}\right| \\
& <\left(1+e^{\operatorname{Re} \lambda}+e^{2 \operatorname{Re} \lambda}\right)^{1 / 2}(r+\delta) \\
& \leq \delta+e^{\operatorname{Re} \lambda} \delta
\end{aligned}
$$

when $\delta \geq \delta_{0}^{\prime}$. This means $e^{\lambda} \cdot \Delta \cap \Delta \neq \emptyset$.

Next we give a condition of the configuration of Peripheral disks.

Proposition 6.7. Under the conditions stated in Proposition 6.5, if $W \in G-H$ satisfies $W^{m}(\Delta(H)) \cap \Delta(H) \neq \emptyset$, then $|m| \leq 1$.

Proof. By Propositions 6.4 and 6.5 there exists $X \in G$ conjugate to $\eta(\mathbf{w}(p / q))$ such that $H \cap W^{m} H W^{-m}=\langle X\rangle$. Notice that $W^{-m} X W^{m} \in H$ and $\Lambda(H) \cap W^{m}(\Lambda(H))$ are the fixed points of $X$. Let $\phi$ be an expanding mapping from $\mathbb{H}^{2}$ to the component $\partial_{0} \mathcal{C}_{\eta}$ of the boundary of the convex full of $\Lambda(G)$ facing $\Omega$. Put $F=\phi^{-1} G \phi$ and let $F_{H}=\phi^{-1} H \phi, \gamma_{1}=\phi^{-1} X \phi, \gamma_{W}=\phi^{-1} W \phi$, and $\gamma_{2}=\gamma_{W}^{-m} \gamma_{1} \gamma_{W}^{m}$. Then these satisfy that $F_{H} \cap \gamma_{W}^{m} F_{H} \gamma_{W}^{-m}=\left\langle\gamma_{1}\right\rangle$ and $\gamma_{2} \in F_{H}$.

Let us assume first that $\gamma_{W}$ is hyperbolic. Let $P_{H}$ be the convex hull of $\Lambda\left(F_{H}\right)$ in $\mathbb{H}^{2}$. Since each axis $\operatorname{Ax}\left(\gamma_{i}\right)$ of $\gamma_{i}$ corresponds to the geodesic homotopic to $\mathbf{w}(p / q)$, these axes are components of the boundary of $P_{H}$.

Here, we claim that the axis of $\gamma_{W}$ intersects both the axes $\operatorname{Ax}\left(\gamma_{1}\right)$ and $\operatorname{Ax}\left(\gamma_{2}\right)$. Indeed, since $\phi\left(P_{H}\right)=\Lambda(H)$ and $\Lambda(H) \cap W^{m}(\Lambda(H))$ is the axis of $X, P_{H} \cap \gamma_{W}^{m}\left(P_{H}\right)$ (resp. $P_{H} \cap \gamma_{W}^{-m}\left(P_{H}\right)$ ) is the axis $\operatorname{Ax}\left(\gamma_{1}\right)$ (resp. $\operatorname{Ax}\left(\gamma_{2}\right)$ ). Hence the fixed points of $\gamma_{W}$ lie on the free sides for $\gamma_{1}$ and $\gamma_{2}$.

Let us prove this proposition. Assume that $m>1$. Denote by $x_{i}$ the intersection point of axes of $\gamma_{W}$ and $\gamma_{i}(i=1,2)$. Since $\gamma_{W}^{m}\left(\operatorname{Ax}\left(\gamma_{1}\right)\right)=\operatorname{Ax}\left(\gamma_{2}\right), \gamma_{W}^{m}\left(x_{1}\right)=x_{2}$. Since $P_{H}$ is convex, the part of the axis of $\gamma_{W}$ between $x_{1}$ and $x_{2}$ is contained in the interior of $P_{H}$. Thus $\gamma_{W}\left(x_{1}\right)$ is contained in the interior of $P_{H}$ and hence $\gamma_{W}\left(P_{H}\right) \cap P_{H}$ contains an open set. This means that the convex hulls of $\Lambda(H)$ and $\Lambda\left(W H W^{-1}\right)$ are contained in the same totally geodesic hemisphere, and hence $W \in H$ by (2) of Proposition 6.2 This contradicts the assumption that $W \in G-H$. If $m<-1$, we may consider $W^{-1}$ instead of $W$ in the argument above.

Finally we shall show that $\gamma_{W}$ is not parabolic by contradiction. Assume that $\gamma_{W}$ is parabolic. We may also assume $\gamma_{W}$ is a parallel translation. Since $W \notin H$ and $H \cap W^{m} H W^{-m}=\langle X\rangle$, by the argument as above, we see that $\gamma_{W}^{m}\left(P_{H}\right)$ and $P_{H}$ intersect at the axis of $\gamma_{1}$. Then by the sublemma below, the axis of $\gamma_{1}$ is a vertical line; hence this contradicts the discreteness of $F_{H}$.

Sublemma. Let $D$ be a closed hyperbolically convex domain in $\mathbb{H}^{2}$ with non-empty interior. Let $T(\zeta)=\zeta+1$ be a translation. If $T(D) \cap D$ is a complete geodesic (that is, a geodesic having infinite length in both directions), it is a vertical line.

Proof. Since $l:=T(D) \cap D$ is complete, $l$ divides the plane $\mathbb{H}^{2}$ into 2 parts, say $E_{1}$ and $E_{2}$. Both $D$ and $T(D)$ have $l$ as a component of their boundaries. Indeed, if some $\zeta \in l$ lies on the interior $\operatorname{Int}(D)$ of $D$, there exists a small ball $D^{\prime}$ in $D$ with center $\zeta$. Since $D^{\prime} \cap T(D) \subset l \cap D^{\prime}$, by the convexity of $T(D)$ and $l \subset T(D)$, a geodesic connecting $\zeta \in l$ and a point of $T(D)$ must lie on $l \cap D^{\prime}$ near $\zeta$. This 
means that $T(D)=l$; this contradicts the assumption that $D$ has non-empty interior. Thus, we may assume that $\operatorname{Int}(D) \subset E_{1}$ and $T(\operatorname{Int}(D)) \subset E_{2}$.

Assume that $l$ is not a vertical line. Let $a, b \in \mathbb{R}$ be end points of $l$. When $|a-b|>1$, then $T(l) \cap l \neq \emptyset$. In this case, it is easy to see that $T(D)$ has an interior point in common with $D$, and this is a contradiction. Hence $|a-b| \leq 1$. By definition of $E_{i}, D$ lies under $l$. Therefore $D$ and $T(D)$ cannot intersect at $l$, which also contradicts the assumption.

Proof of Proposition 6.6. Let $\mathfrak{a}_{0}$ be a universal constant given in Lemma 6.3 Then we shall see that the constant

$$
\delta_{0}=\delta_{0}(\operatorname{Re} \lambda, r)=\max _{2 \leq a \leq \mathfrak{a}_{0}}\left\{\frac{r\left(1+e^{a \operatorname{Re} \lambda}+e^{2 a \operatorname{Re} \lambda}\right)^{1 / 2}}{1+e^{a \operatorname{Re} \lambda}-\left(1+e^{a \operatorname{Re} \lambda}+e^{2 a \operatorname{Re} \lambda}\right)^{1 / 2}}\right\}
$$

is a desired one.

Indeed, let $\Delta$ be a peripheral disk which intersects a disk $\{|\zeta|<r\}$. By Lemma 6.3, there exist integers $a$ and $c$ such that $2 \leq a \leq \mathfrak{a}_{0}$ and that $|a(\operatorname{Im} \lambda / \pi)-2 c|<$ $1 / 3$. Since $a \geq 2, W^{a}(\Delta)$ cannot intersect $\Delta$ by Proposition 6.7 Hence by Lemma 6.4, the radius of $\Delta$ is less than $\delta_{0}$.

\section{DERIVATIVE IS OF LINEAR ORDER}

In this section, we will introduce three theorems, Theorems [9, 11, and 12. The first theorem implies our main theorem of this paper (Theorem 2). The next two theorems are used to show the first.

7.1. Statement of the theorem 'Derivative is of linear order'. Let us continue to use the notation defined in previous sections. Let $p / q \in \mathbb{Q} \mathcal{X}$. Denote by $\lambda_{p / q}(x)$ the complex length of $X_{p / q, x}$, that is, $\lambda_{p / q}$ satisfies the equation

$$
\operatorname{tr}^{2} X_{p / q, x}=4 \cosh ^{2}\left(\lambda_{p / q}(x) / 2\right) .
$$

We take $\lambda_{p / q}$ to be holomorphic on $\mathcal{X}$ and satisfying that $\operatorname{Re} \lambda_{p / q}(x)>0$ on $\mathcal{X}$ and $\lambda_{p / q}(x) \in \mathbb{R}$ for any point on $\mathcal{P}_{p / q}^{\mathcal{X}}$.

In this and the following sections, we fix a rational number $\mathfrak{q} \in \mathbb{Q} \mathcal{X}$. Notice that for $p / q \in \mathbb{Q} \mathcal{X}-\{\mathfrak{q}\}, \lambda_{p / q}$ can be extended holomorphically on a neighborhood of $x(\mathfrak{q})$ because $X_{p / q, x}$ is either loxodromic or hyperbolic at $x(\mathfrak{q})$. Denote by $\ell(x)$ the restriction of $\lambda_{\mathfrak{q}}$ to $\mathcal{P}_{\mathfrak{q}}^{\mathcal{X}}$. By Proposition 5.1, $\ell(x)$ is a real analytic diffeomorphism of $\mathcal{P}_{\mathfrak{q}}^{\mathcal{X}}$ onto its image. Here we state the following.

Theorem 9 (Derivative is of linear order). Suppose that $\mathcal{X}=\mathcal{M}$ or $\mathcal{B}_{G}$ for some quasifuchsian group $G$ of type $(1,1)$. Then for $p / q \in \mathbb{Q}_{\mathcal{X}}-\{\mathfrak{q}\}$,

$$
\left|\frac{d}{d l}\left(\lambda_{p / q} \circ \ell^{-1}\right)(l)\right| \lesssim l, \text { as } l \rightarrow 0
$$

where the implicit constant is dependent only on the translation length of $X_{p / q, x}$ at $x=x(\mathfrak{q})$, either when $\mathcal{X}=\mathcal{M}$ or when $\mathcal{X}=\mathcal{B}_{G}$ and $\mathbf{w}(\mathfrak{q})$ does not correspond to one of the shortest geodesics on a hyperbolic surface $\left(\Sigma, \nu_{-}\left(\rho_{0}^{b}\right)\right)$. Otherwise, the constant, in addition, depends on the length of the geodesic corresponding to $\mathbf{w}(\mathfrak{q})$ on $\left(\Sigma, \nu_{-}\left(\rho_{0}^{b}\right)\right)$, where the $\nu_{-}\left(\rho_{0}^{b}\right)$ is the negative end invariant of $\rho_{x}^{b}$ at $x=0 \in \mathcal{B}_{G}$ (see Section [3). 
The proof of Theorem 9 is given in Section 7.4 after stating and assuming Theorems 11$]$ and 12 These two theorems are proved in Section 8. To state the two theorems, we begin with the following formula.

7.2. Parker and Series' bending formula. The content of this section is due to Parker-Series [52].

Let $\eta$ be a once punctured torus group with distinguished invariant component $\Delta$. Assume that $\partial_{0} \mathcal{C}_{\eta}$ is bent along the geodesic corresponding to $\mathbf{w}(p / q)$ for some $p / q$. Put $X=\eta(\mathbf{w}(p / q))$ and $G=\eta\left(\pi_{1}\right)$. Take $V \in G$ so that $G=\langle X, V\rangle$.

Let us recall the definition of the complex shear. Let $\xi$ be the oriented common perpendicular from $\mathrm{Ax} X$ to $\mathrm{Ax} V^{-1} X V$. Then the complex shear is defined to be $\pm d$ where $d$ is the complex distance from $\xi$ to $V(\xi)$. The sign is determined by the condition $\operatorname{Im}( \pm d) \in[0, \pi]$. We do not give the concrete definition of the complex length here, but we should know that the imaginary part of the complex shear coincides with the bending angle.

Denote by $\lambda(W)$ the complex length of $W$ for a loxodromic element $W$. Then the relation between the complex shear and $\lambda(X)$ and $\lambda(V)$ is given as follows:

Theorem 10 (Bending formula). Denote by $\tau$ the complex shear. Then

$$
\cosh ^{2}(\tau / 2)=\cosh ^{2}(\lambda(V) / 2) \tanh ^{2}(\lambda(X) / 2) .
$$

Relation between length and bending angle. For $x \in \mathcal{P}_{\mathfrak{q}}^{\mathcal{X}}$, define $\theta(x) \in \mathbb{R}$ so that $\pi-\theta(x)$ is the bending angle.

Lemma 7.1. We have $\theta(x) \lesssim \ell(x)$ as $x \rightarrow x(\mathfrak{q})$ and $x \in \mathcal{P}_{p / q}^{\mathcal{X}}$. Furthermore, the implicit constant is a universal constant, either when $\mathcal{X}=\mathcal{M}$ or when $\mathcal{X}=\mathcal{B}_{G}$ and $\mathbf{w}(\mathfrak{q})$ does not correspond to the shortest geodesic on $\left(\Sigma, \nu_{-}\left(\rho_{0}^{b}\right)\right)$. Otherwise, the implicit constant is dependent only on the length of the shortest geodesic on $\left(\Sigma, \nu_{-}\left(\rho_{0}^{b}\right)\right)$.

Proof. Fix $p^{\prime} / q^{\prime} \in \mathbb{Q}_{\mathcal{X}}$ so that $\pi_{1}=\left\langle\mathbf{w}(\mathfrak{q}), \mathbf{w}\left(p^{\prime} / q^{\prime}\right)\right\rangle$. Let $\tau(x)$ be the complex shear defined for $X=X_{\mathfrak{q}, x}$ and $V=X_{p^{\prime} / q^{\prime}, x}$. Then the Bending formula gives that

$$
\begin{aligned}
\sin ^{2}(\theta(x) / 2) & =\cos ^{2}(\operatorname{Im} \tau(x) / 2) \leq\left|\cosh ^{2}(\tau(x) / 2)\right| \\
& =\mid \cosh ^{2}\left(\lambda_{p^{\prime}} / q^{\prime}\right. \\
& =4^{-1}\left|\operatorname{tr}^{2} X_{p^{\prime} / q^{\prime}, x}\right| \tanh ^{2}(\ell(x) / 2) .
\end{aligned}
$$

Since the trace function $\operatorname{tr}^{2} X_{p^{\prime} / q^{\prime}, x}$ is holomorphic at $x=x(\mathfrak{q})$ and $\sin \theta \asymp \theta$ and $\tanh l \asymp l$ as $\theta, l \rightarrow 0$ hold, the first part of the assertion is established. The second part of this lemma follows from the following proposition.

Proposition 7.1. Suppose that $\mathcal{X}=\mathcal{B}_{G}$ or $\mathcal{M}$. Let $\mathfrak{q} \in \mathbb{Q} \mathcal{X}$. Then, there exists a constant $\kappa_{1}>0$ and $p^{\prime} / q^{\prime} \in \mathbb{Q}_{\mathcal{X}}$ such that

(i) the geometric intersection number between $\mathbf{w}(\mathfrak{q})$ and $\mathbf{w}\left(p^{\prime} / q^{\prime}\right)$ is one, and

(ii) at $x=x(\mathfrak{q}), X_{p^{\prime} / q^{\prime}, x}$ is loxodromic and $\left|\operatorname{tr}^{2} X_{p^{\prime} / q^{\prime}, x}\right| \leq \kappa_{1}$.

In addition, the constant $\kappa_{1}$ is a universal constant, either when $\mathcal{X}=\mathcal{M}$ or when $\mathcal{X}=\mathcal{B}_{G}$ and $\mathbf{w}(\mathfrak{q})$ does not correspond to the shortest geodesic on $\left(\Sigma, \nu_{-}\left(\rho_{0}^{b}\right)\right)$. Otherwise, the constant is dependent only on the length of the shortest geodesic on $\left(\Sigma, \nu_{-}\left(\rho_{0}^{b}\right)\right)$. 


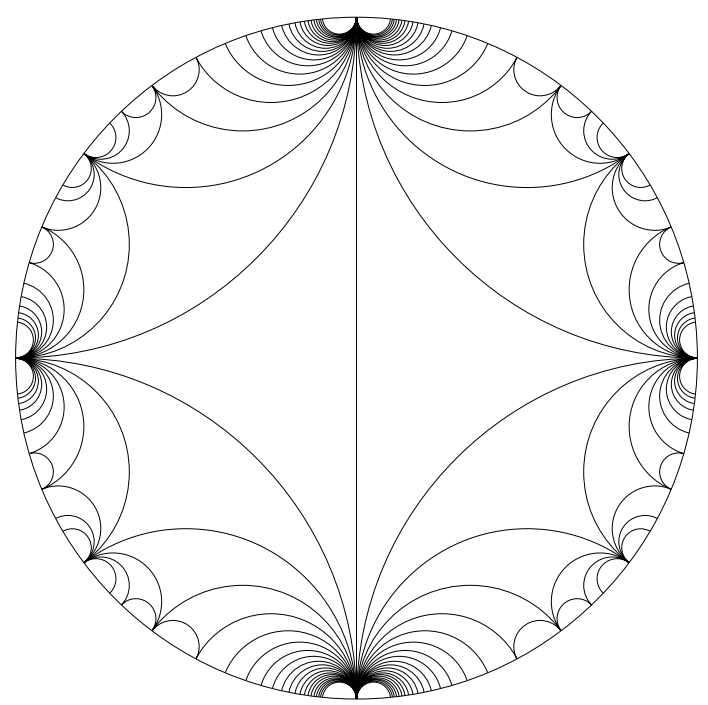

FiguRE 6. Farey triangulation

To prove this, let us first recall the Farey triangulation of $\mathbb{H}$ (see Figure [6) and its geometric properties as the partition of Teichmüller space (cf. Section 2 of [47]). The Farey triangulation is a triangulation by ideal triangles in $\mathbb{H}$ whose vertices consist of $\widehat{\mathbb{Q}} \subset \partial \mathbb{H}$, and two rational numbers $p / q, p^{\prime} / q^{\prime}$ are connected by the hyperbolic geodesic connecting them only when $\left|p q^{\prime}-p^{\prime} q\right|=1$, equivalently, when the geometric intersection number of corresponding curves $\mathbf{w}(p / q)$ and $\mathbf{w}\left(p^{\prime} / q^{\prime}\right)$ is one. We denote by $\mathcal{K}$ the 1 -skeleton of this triangulation.

We now identify the upper half plane as Teichmüller space as in Section 2.2. It is known that for $\nu_{0} \in \mathbb{H}$, the geodesic homotopic to $\mathbf{w}(1 / 0)$ on a hyperbolic surface $\left(\Sigma, \nu_{0}\right)$ is the shortest one if and only if $\left|\nu_{0}-n\right|>1$ for all $n \in \mathbb{Z}$. Hence if a point $\mathfrak{p} \in \hat{\mathbb{Q}}$ corresponds to one of the shortest curves of a hyperbolic surface $(\Sigma, \nu)$ $(\nu \in \mathbb{H}), \nu$ is contained in an ideal triangle with vertex $\mathfrak{p}$.

Let $\eta$ be a once punctured torus group. Let us denote by $\mathcal{K}(\eta, \kappa)$ the subgraph of $\mathcal{K}$ spanned by rational numbers $\mathfrak{p}$ with the property that the translation length $\eta(\mathbf{w}(\mathfrak{p}))$ is less than $\kappa>0$. Y. Minsky [47] showed that there exists a universal constant $\kappa_{2}$ so that $\mathcal{K}\left(\eta, \kappa_{2}\right)$ is connected and that any shortest curve on any hyperbolic once punctured torus has length less than $\kappa_{2}$.

Proof of Proposition 7.1. Let us prove Proposition 7.1. The argument is an analog of that of Lemma 8.2 of [47. We first define $\mathfrak{p} \in \mathbb{Q} \mathcal{X}$ as follows (see Figure 7):

(1) In the case where $\mathcal{X}=\mathcal{B}_{G}$ and $\mathbf{w}(\mathfrak{q})$ does not correspond to one of the shortest curves on a hyperbolic surface $\left(\Sigma, \nu_{-}\left(\rho_{0}^{b}\right)\right)$, we take $\mathfrak{p}$ so that $\mathbf{w}(\mathfrak{p})$ represents one of the shortest simple closed geodesics on $\left(\Sigma, \nu_{-}\left(\rho_{0}^{b}\right)\right)$.

(2) In the case where $\mathcal{X}=\mathcal{B}_{G}$ and $\mathbf{w}(\mathfrak{q})$ represents one of the shortest curves on a hyperbolic surface $\left(\Sigma, \nu_{-}\left(\rho_{0}^{b}\right)\right)$, we take $\mathfrak{p}$ as an another vertex of the ideal triangle containing $\nu_{-}\left(\rho_{0}^{b}\right)$ rather than $\mathfrak{q}$.

(3) When $\mathcal{X}=\mathcal{M}$, we put $\mathfrak{p}=1 / 0$. 


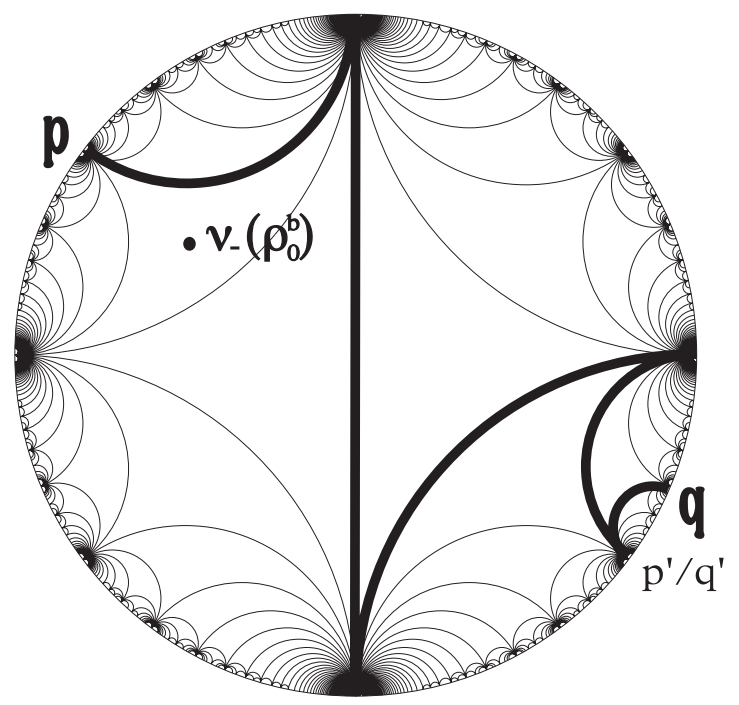

FiguRE $7 \cdot \mathfrak{p}, \mathfrak{q}$, and $p^{\prime} / q^{\prime}$

Notice here that in the case of $\mathcal{X}=\mathcal{B}_{G}, \nu_{-}\left(\rho_{x}^{b}\right)=\nu_{-}\left(\rho_{0}^{b}\right)$ for all $x \in \overline{\mathcal{B}_{G}}$.

We first treat the case (1). Since $\mathbf{w}(\mathfrak{p})$ corresponds to one of the shortest geodesics on $\left(\Sigma, \nu_{-}\left(\rho_{x(\mathfrak{q})}^{b}\right)\right), \mathfrak{p} \in \mathcal{K}\left(\rho_{x(\mathfrak{q})}^{b}, \kappa_{2}\right)$ by definition. Since $X_{\mathfrak{q}, x(\mathfrak{q})}$ is parabolic, $\mathfrak{q} \in \mathcal{K}\left(\rho_{x(\mathfrak{q})}^{b}, \kappa_{2}\right)$. Hence we can connect two points $\mathfrak{q}$ and $\mathfrak{p}$ by the path in $\mathcal{K}\left(\rho_{x(\mathfrak{q})}^{b}, \kappa_{2}\right)$ by connectivity. Denote by $p^{\prime} / q^{\prime}$ the next of $\mathfrak{q}$ in the path. Then, by definition, $X_{p^{\prime} / q^{\prime}, x(\mathfrak{q})}$ is loxodromic, the geometric intersection number of $\mathbf{w}(\mathfrak{q})$ and $\mathbf{w}\left(p^{\prime} / q^{\prime}\right)$ is one, and its translation length is less than $\kappa_{2}$. Therefore, the absolute value of the trace of $X_{p^{\prime} / q^{\prime}, x(\mathfrak{q})}$ is dominated by a universal constant $4 \cosh ^{2}\left(\kappa_{2} / 2\right)$.

In case (2), we may put $p^{\prime} / q^{\prime}=\mathfrak{p}$. Indeed, since $p^{\prime} / q^{\prime}$ and $\mathfrak{q}$ consist of the end points of common edge, the geometric intersection number of the corresponding curves is one. Furthermore, since $p^{\prime} / q^{\prime}$ and $\mathfrak{q}$ are vertices of the ideal triangle containing $\nu_{-}\left(\rho_{0}^{b}\right)$, the length of the closed geodesic on $\left(\Sigma, \nu_{-}\left(\rho_{0}^{b}\right)\right)$ homotopic to $\mathbf{w}(\mathfrak{p})$ depends only on the length of that on the same surface homotopic to $\mathbf{w}(\mathfrak{q})$. Hence the translation length of $X_{p^{\prime} / q^{\prime}, x(\mathfrak{q})}$ is also dependent only on the length of the closed geodesic on $\left(\Sigma, \nu_{-}\left(\rho_{0}^{b}\right)\right)$ homotopic to $\mathbf{w}(\mathfrak{q})$ by Bers inequality (cf. Theorem 3 of [5]). Therefore so is the absolute value of the trace of $X_{p^{\prime}} / q^{\prime}, x(\mathfrak{q})$.

Finally, we consider case (3). If the geometric intersection number between $\mathbf{w}(\mathfrak{q})$ and $\mathbf{w}(1 / 0)$ is greater than one, we can find appropriate $p^{\prime} / q^{\prime}$ by the same argument as that of case (1). Otherwise, $\mathfrak{q}$ is equal to $n / 1$ for some $n \in \mathbb{Z}$. Then the rational number $p^{\prime} / q^{\prime}=(n+1) / 1$ is the desired one, since $x^{m}(n / 1)=2 n+2 i$ and $\operatorname{tr}^{2} X_{(n+1) / 1, x}=-(x-2(n+1))^{2}$.

7.3. Quasiconformal deformation. We define a quasiconformal deformation of groups on a pleating ray. This is the central tool for proving Theorem 9 (Derivative is of linear order).

Let $\ell$ and $\theta$ be as in the previous sections. Let $x \in \mathcal{P}_{\mathfrak{q}}^{\mathcal{X}}$. Let $H_{1}$ and $H_{2}$ be the peripheral subgroups containing $X_{\mathfrak{q}, x}$. Since $H_{i}$ acts on $\Delta\left(H_{i}\right)$, we can consider the axis $\sigma_{i}$ of $X_{\mathfrak{q}, x}$ in $\Delta\left(H_{i}\right)$ as in 2-dimensional hyperbolic geometry. Each $\sigma_{i}$ is 


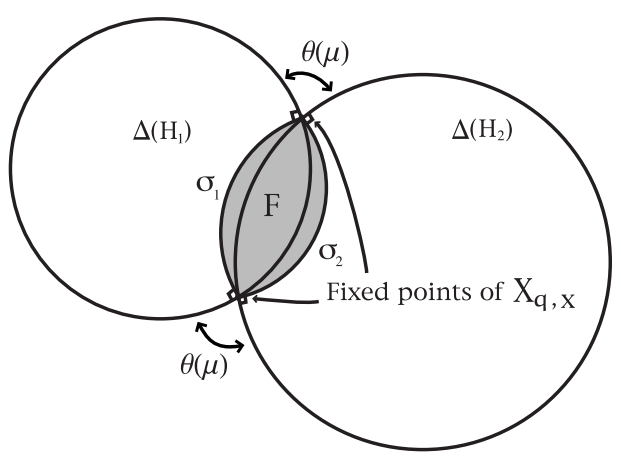

Figure 8. The set $F$

a circular arc orthogonal to $\partial \Delta\left(H_{i}\right)$ at the fixed points of $X_{\mathfrak{q}, x}$, and $\sigma_{1}$ and $\sigma_{2}$ bound the sector $F$ contained in $\Delta\left(H_{1}\right) \cup \Delta\left(H_{2}\right)$. This $F$ is uniquely determined (see Figure 8).

Set $F_{[B]}=B^{-1}(F)$ for $[B] \in\left\langle X_{\mathfrak{q}, x}\right\rangle \backslash G_{x}$. (Notice that $F$ is invariant under the action of $X_{\mathfrak{q}, x}$.) Then, for $\left[B_{1}\right],\left[B_{2}\right] \in\left\langle X_{\mathfrak{q}, x}\right\rangle \backslash G_{x}, F_{\left[B_{1}\right]} \cap F_{\left[B_{2}\right]}=\emptyset$ if $\left[B_{1}\right] \neq\left[B_{2}\right]$.

Fix a Möbius transformation $A$ sending the fixed points of $X_{\mathfrak{q}, x}$ to $\{0, \infty\}$. Then $A(F)$ becomes a sector with center at origin whose central angle is $\pi-\theta(x)$. Set $\hat{\tau}(\zeta)=A(\zeta) \overline{A^{\prime}(\zeta)} / \overline{A(\zeta)} A^{\prime}(\zeta)$ on $F$ and $\hat{\tau}(\zeta)=0$ otherwise. Define the Beltrami differential $\tau_{x}, x \in \mathcal{P}_{\mathfrak{q}}^{\mathcal{X}}$ by

$$
\tau_{x}(\zeta)=\frac{1}{\ell(x)} \sum_{[B] \in\left\langle X_{\mathfrak{q}, x}\right\rangle \backslash G_{x}} \hat{\tau}(B(\zeta)) \overline{\frac{\partial B}{\partial \zeta}(\zeta)}\left(\frac{\partial B}{\partial \zeta}(\zeta)\right)^{-1} .
$$

The differential $\tau_{x}$ satisfies $\tau_{x} \circ g \cdot \overline{g^{\prime}}=\tau_{x} \cdot g^{\prime}$ for all $g \in G_{x}$. Furthermore, $\left\|\tau_{x}\right\|_{\infty}=$ $1 / \ell(x)$ and the support of $\tau_{x}$ is $\bigcup_{[B] \in\left\langle X_{\mathfrak{q}, x}\right\rangle \backslash G_{x}} F_{[B]}$.

For $t \in \mathbb{C}$ with $|t|<\ell(x)$, let $w^{t}$ be a solution (depending holomorphically on $t$ ) on $\hat{\mathbb{C}}$ of the Beltrami equation $\bar{\partial} w^{t}=t \tau_{x} \partial w^{t}$. This deformation defines a holomorphic mapping $\Phi_{x}$ from a disk $\{|t|<\ell(x)\}$ into $\mathcal{X}$ such that $\Phi_{x}(0)=x$ and $G_{\Phi_{x}(t)}$ is conjugate to $w^{t} G_{x}\left(w^{t}\right)^{-1}$.

\subsection{Proof of the theorem 'Derivative is of linear order'.}

Theorem 11. Let $\tau_{x}$ be the Beltrami differential defined as above. Suppose that $\mathcal{X}=\mathcal{M}$ or $\mathcal{B}_{G}$ for some $G=G\left(z_{0}, y_{0}\right)$. Then there exists $l_{1}>0$ so that

$$
\left|d \lambda_{\mathfrak{q}}\left[\tau_{x}\right]\right|>1 / 2 \text { for } 0<l(x)<l_{1} .
$$

Theorem 12 (Remaining curves are not so deformed). Suppose that $\mathcal{X}=\mathcal{M}$ or $\mathcal{B}_{G}$ for some $G=G\left(z_{0}, y_{0}\right)$. Then for $p / q \in \mathbb{Q} \mathcal{X}-\{\mathfrak{q}\}$,

$$
\left|d \lambda_{p / q}\left[\tau_{x}\right]\right| \lesssim \ell(x) \text { as } x \rightarrow x(\mathfrak{q}) .
$$

Moreover the implicit constant is dependent only on the translation length of $X_{p / q, x}$ at $x=x(\mathfrak{q})$, either when $\mathcal{X}=\mathcal{M}$ or when $\mathcal{X}=\mathcal{B}_{G}$ and $\mathbf{w}(\mathfrak{q})$ does not correspond to the shortest geodesic on $\left(\Sigma, \nu_{-}\left(\rho_{0}^{b}\right)\right)$. Otherwise, the implicit constant is also dependent on the length of the shortest geodesic on $\left(\Sigma, \nu_{-}\left(\rho_{0}^{b}\right)\right)$.

Theorems 11 and 12 are proved in Sections 8 and 10 respectively. For the rest of this subsection, we shall show that these two theorems imply Theorem 9 
Indeed, take $l>0$ sufficiently small and let $x \in \mathcal{P}_{\mathfrak{q}}^{\mathcal{X}}$ with $\ell(x)=l$. Let $\Phi_{x}$ be the holomorphic mapping of the disk $\{|t|<\ell(x)\}$ to $\mathcal{X}$ as defined in Section 7.3. From Theorem 11, we may suppose that $\Phi_{x}$ and $\lambda_{\mathfrak{q}} \circ \Phi_{x}$ are both conformal at $t=0$. Since the restriction of $\lambda_{\mathfrak{q}}$ on $\mathcal{P}_{\mathfrak{q}}^{\mathcal{X}}$ agrees with $\ell(x)$ and $\Phi_{x}(0)=x$, we obtain

$$
\begin{aligned}
\left|\frac{d}{d l}\left(\lambda_{p / q} \circ \ell^{-1}\right)(l)\right| & =\left.\left|\frac{d}{d t}\left(\lambda_{p / q} \circ \Phi_{x}\right)\right|_{t=0}|\cdot| \frac{d}{d l}\left(\Phi_{x}^{-1} \circ \ell^{-1}\right)\right|_{l} \mid \\
& =\left|d \lambda_{p / q}\left[\tau_{x}\right]\right| \cdot\left|d \lambda_{\mathfrak{q}}\left[\tau_{x}\right]\right|^{-1} \lesssim l .
\end{aligned}
$$

\section{Differential ON A PINCHED CURVE UNDER QC-DEFORMATion}

8.1. Gardiner's formula. We review the differential formula for the complex length of loxodromic elements under quasiconformal deformations.

Let $g(\zeta)=e^{\lambda} \zeta$ with $\operatorname{Re} \lambda>0$. Let $\tau$ be a Beltrami differential on $\mathbb{C}$ compatible with $g$. We denote by $f^{t}$ a solution on $\hat{\mathbb{C}}$ of the equation $\bar{\partial} f^{t}=t \nu \partial f^{t}$ for $|t|<$ $1 /\|\tau\|_{\infty}$. Define the holomorphic function $\lambda(t)$ on $\{|t|<1 /\|\tau\|\}$ by $\operatorname{tr}^{2} f^{t} g\left(f^{t}\right)^{-1}=$ $4 \cosh ^{2}(\lambda(t) / 2)$ and $\lambda(0)=\lambda$.

Proposition ([16]). Under the notation above,

$$
\left.\frac{d \lambda}{d t}\right|_{t=0}=\frac{1}{\pi} \int_{\left\{1<|\zeta|<e^{\operatorname{Re} \lambda}\right\}} \tau(\zeta) \frac{d u d v}{\zeta^{2}}, \quad \zeta=u+i v .
$$

Indeed, F. Gardiner proved this formula for fuchsian groups. However, his proof can be applied to the cases of general Kleinian groups.

8.2. Proof of Theorem 11. By Lemma [.1 there exists $l_{0}>0$ so that for $x \in \mathcal{P}_{\mathfrak{q}}^{\mathcal{X}}$ with $\ell(x)<l_{0}$, the inequality $\theta(x)<\pi / 2$ holds. For such $x \in \mathcal{P}_{\mathfrak{q}}^{\mathcal{X}}$, we take a conjugation $G_{x}=\eta_{x}\left(\pi_{1}\right)$ by a Möbius transformation sending the fixed points of $X_{\mathfrak{q}, x}$ to $\{0, \infty\}$. To avoid confusion, we use the same symbols to represent $G_{x}, X_{\mathfrak{q}, x}$, etc. Here, we may suppose that $X_{\mathfrak{q}, x}(\zeta)=e^{\ell(x)} \zeta, \Delta\left(H_{1}\right)=\mathbb{H}$, and $\Delta\left(H_{1}\right) \cap \Delta\left(H_{2}\right) \subset\{z \mid \operatorname{Re} z>0\}$, where $H_{1}$ and $H_{2}$ are the peripheral subgroups of $G_{x}$ containing $X_{\mathfrak{q}, x}$.

Since $\operatorname{tr}^{2} X_{\mathfrak{q}, \Phi_{x}(t)}=\operatorname{tr}^{2} w^{t} X_{\mathfrak{q}, x}\left(w^{t}\right)^{-1}$ and $\lambda_{\mathfrak{q}} \circ \Phi_{x}(0)=\ell(x)$, it follows from Gardiner's formula that

$$
d \lambda_{\mathfrak{q}}\left[\tau_{x}\right]=\left.\frac{d}{d t}\left(\lambda_{\mathfrak{q}} \circ \Phi_{x}\right)\right|_{t=0}=\frac{1}{\pi} \int_{\mathcal{A}_{x}} \tau_{x}(\zeta) \frac{d u d v}{\zeta^{2}}
$$

where $\zeta=u+i v$ and $\mathcal{A}_{x}=\left\{\zeta \in \mathbb{C}|1<| \zeta \mid<e^{\ell(x)}\right\}$. By definition, $F=$ $\{\pi / 2<\arg \zeta<3 \pi / 2-\theta(x)\}$ and $\tau_{x}=\zeta / \ell(x) \bar{\zeta}$ on $F$. Let $D_{1}=F \cap \mathcal{A}_{x}$ and $D_{2}=\left(\operatorname{Supp}\left(\tau_{x}\right) \cap \mathcal{A}_{x}\right) \backslash D_{1}$ (see Figure 9). We denote by $J_{k}, k=1,2$ the integral over $D_{k}$. Then the integral in (6) is equal to $J_{1}+J_{2}$. A simple calculation shows that $J_{1}=(\pi-\theta(x)) / \pi$.

Let us consider the integral $J_{2}$. We define $\vartheta(x)>0$ by $\tan \vartheta(x)=1 / \sinh (\ell(x) / 2)$. Then Keen-Halpern's collar lemma asserts that the sector $Y_{1}=\{\pi / 2-\vartheta(x)<$ $\arg \zeta \leq \pi / 2\}$ is disjoint from lifts of boundary geodesics of $\Delta\left(H_{1}\right) / H_{1}$ with the exception of the axis of $X_{\mathfrak{q}, x}$ in $\Delta\left(H_{1}\right)$, the positive imaginary axis. This implies that $Y_{1} \cap D_{2}=\emptyset$. One shows in the same way that the sector $Y_{2}=\{3 \pi / 2-\theta(x) \leq$ $\arg \zeta<3 \pi / 2-\theta(x)+\vartheta(x)\}$ is disjoint from $D_{2}$. Hence $D_{2}$ is contained in the sector 

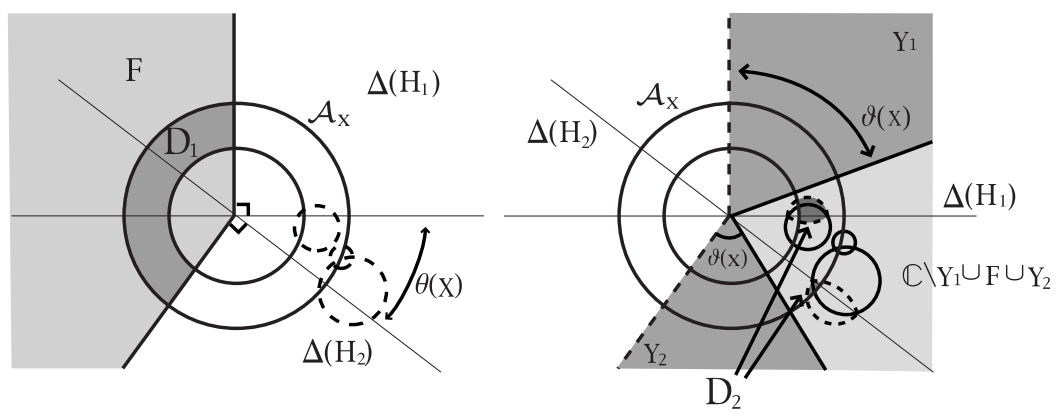

FiguRE $9 . D_{1}$ and $D_{2}$

$\mathbb{C} \backslash\left(Y_{1} \cup F \cup Y_{2}\right)$ and the annulus $\mathcal{A}_{x}$. This implies $\left|J_{2}\right|<(\pi-2 \vartheta(x)+\theta(x)) / \pi(\mathrm{cf}$. Figure 9). Thus we have

$$
\left|d \lambda_{\mathfrak{q}}\left[\tau_{x}\right]\right| \geq\left|J_{1}\right|-\left|J_{2}\right|>2(\vartheta(x)-\theta(x)) / \pi \rightarrow 1 \text { as } x \rightarrow x(\mathfrak{q})
$$

by the definition of $\vartheta(x)$ and Lemma 7.1

\section{Differential on Remaining CURVES UNDER QC-DEFORMation}

Let $p / q \in \mathbb{Q}_{\mathcal{X}}-\{\mathfrak{q}\}$. As in the proof of Theorem 11, we use the same notation to represent $G_{x}$ and $X_{p / q, x}$, and so forth. Here we take a conjugation of $G_{x}$ for each $x \in \mathcal{P}_{\mathfrak{q}}^{\mathcal{X}}$ such that the fixed points of $X_{p / q, x}$ map to $\{0, \infty\}$, and hence $X_{p / q, x}(\zeta)$ forms the expansion $\zeta \mapsto e^{\lambda_{p / q}(x)} \zeta$ with $\operatorname{Re} \lambda_{p / q}(x)>0$.

9.1. Proof of Theorem $\mathbf{1 2}$ (Remaining curves are not so deformed). Take $l_{2}>0$ so that $\theta(x)<\pi / 4$ and $\operatorname{Re} \lambda_{p / q}(x)<2 \operatorname{Re} \lambda_{p / q}(x(\mathfrak{q}))$ if $\ell(x)<l_{2}$. We may suppose in addition that $l_{2}<\kappa_{0}$ where $\kappa_{0}$ is defined in Proposition 6.5 for $\epsilon_{2}=3 \pi / 4$. Fix a point $x$ with such properties.

Let $[B] \in\left\langle X_{\mathfrak{q}, x}\right\rangle \backslash G_{x}$. We suppose that $B^{-1} X_{\mathfrak{q}, x} B(\zeta)=\left(a_{B} \zeta+b_{B}\right) /\left(c_{B} \zeta+d_{B}\right)$ with $a_{B} d_{B}-b_{B} c_{B}=1$. Then it follows from $\theta(x)<\pi / 2$ that $F_{[B]}$ is contained in the disk with center $\left(a_{B}-d_{B}\right) / 2 c_{B}$ and of radius $\sinh (\ell(x) / 2) /\left|c_{B}\right|$. This implies

$$
\operatorname{Area}\left(F_{[B]}\right) \leq \pi \sinh ^{2}(\ell(x) / 2) /\left|c_{B}\right|^{2} \lesssim \ell(x)^{2}\left|c_{B}\right|^{-2},
$$

where Area $(-)$ means the 2-dimensional Lebesgue measure.

Recall that the support of $\tau_{x}$ is the disjoint union of the sets $\left\{F_{[B]}\right\},[B] \in$ $\left\langle X_{\mathfrak{q}, x}\right\rangle \backslash G_{x}$ (cf. Section [7.3). Applying Gardiner's formula again, we have

$$
\begin{aligned}
\left|d \lambda_{p / q}\left[\tau_{x}\right]\right| & =\left|\frac{d}{d t} \lambda_{p / q} \circ \Phi_{x}\right|_{t=0} \mid \\
& =\left|\frac{1}{\pi} \int_{\left\{1<|\zeta|<e^{\operatorname{Re} \lambda_{p / q}(x)}\right\}} \tau_{x}(\zeta) \frac{d u d v}{\zeta^{2}}\right| \\
& \leq \frac{1}{\pi} \int_{\left\{|\zeta| \leq 2 \operatorname{Re} \lambda_{p / q}(x(\mathfrak{q}))\right\}}\left|\tau_{x}(\zeta)\right| d u d v \\
& \leq \frac{1}{\pi \ell(x)} \sum_{[B]}^{\prime} \operatorname{Area}\left(F_{[B]}\right) \lesssim \ell(x) \sum_{[B]}^{\prime}\left|c_{B}\right|^{-2},
\end{aligned}
$$



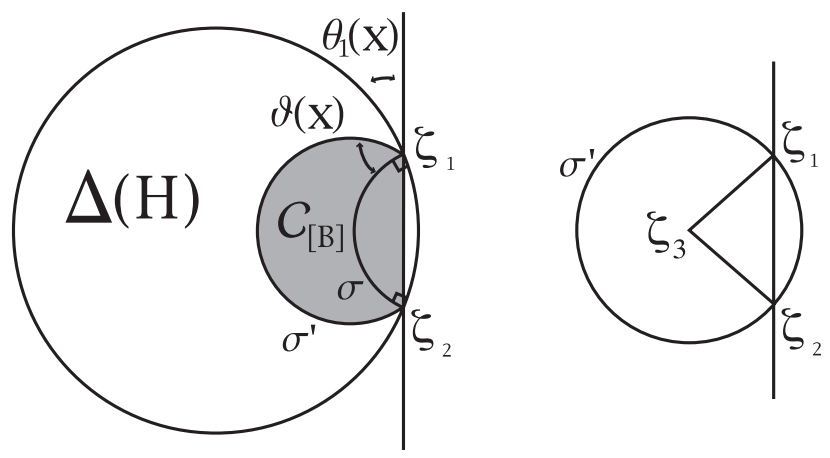

Figure 10. The domain $\mathcal{C}_{[B]}$

where $\sum_{[B]}^{\prime}$ means the summation over all $[B] \in\left\langle X_{\mathfrak{q}, x}\right\rangle \backslash G_{x}$ with $F_{[B]} \cap\{|\zeta| \leq$ $\left.2 \operatorname{Re} \lambda_{p / q}(x(\mathfrak{q}))\right\} \neq \emptyset$. Thus, Theorem 12 follows from Proposition 9.1 which is proved below.

Proposition 9.1. With notation as above, we have

$$
\sum_{[B]}^{\prime}\left|c_{B}\right|^{-2}=O(1)
$$

as $\ell(x) \rightarrow 0$, where the right-hand side of the inequality above depends only on the translation length $\operatorname{Re} \lambda_{p / q}(x(\mathfrak{q}))$ of $X_{p / q, x(\mathfrak{q})}$, either when $\mathcal{X}=\mathcal{M}$ or when $\mathcal{X}=\mathcal{B}_{G}$ and $\mathbf{w}(\mathfrak{q})$ does not correspond to the shortest geodesic on a hyperbolic surface $\left(\Sigma, \nu_{-}\left(\rho_{0}^{b}\right)\right), 0 \in \mathcal{B}_{G}$. Otherwise, it also depends on the length of the shortest geodesic on $\left(\Sigma, \nu_{-}\left(\rho_{0}^{b}\right)\right)$.

Proof. In this proof, we frequently use the symbols defined in the proof of Theorem 12 above.

Let $[B]$ be an element in $\left\langle X_{\mathfrak{q}, x}\right\rangle \backslash G_{x}$ with $F_{[B]} \cap\left\{|\zeta| \leq 2 \operatorname{Re} \lambda_{p / q}(x(\mathfrak{q}))\right\} \neq \emptyset$. Fix a peripheral subgroup $H$ of $G_{x}$ containing $B^{-1} X_{\mathfrak{q}, x} B$. Let $\sigma$ be the axis of $B^{-1} X_{\mathfrak{q}, x} B$ in $\Delta(H)$ considered as the 2-dimensional hyperbolic disk. Let $\left\{\zeta_{k}\right\}_{k=1,2}$ be the fixed point of $B^{-1} X_{\mathfrak{q}, x} B$. We denote by $N_{H}$ the Nielsen region of $H$ in $\Delta(H)$. Then

$$
\sigma^{\prime}:=\left\{z \in N_{H} \mid \sinh \left(d_{\Delta(H)}(z, \sigma)\right) \sinh (\ell(x) / 2)=1\right\}
$$

is a circular arc in $\Delta(H)$ whose end points are $\left\{\zeta_{k}\right\}_{k=1,2}$, where $d_{\Delta(H)}$ means the hyperbolic distance on $\Delta(H)$. Therefore, as in Figure 10, the curve consisting of $\sigma^{\prime}$ and the line segment joining $\zeta_{1}$ and $\zeta_{2}$ bounds a domain $\mathcal{C}_{[B]}$ in $\Delta(H)$. KeenHalpern's collar lemma asserts that the sets $\mathcal{C}_{[B]}$ are pairwise disjoint for $[B] \in$ $\left\langle X_{\mathfrak{q}, x}\right\rangle \backslash G_{x}$.

Let us define a continuous function $\vartheta(x)$ on $\mathcal{P}_{\mathfrak{q}}^{\mathcal{X}} \cup\{x(\mathfrak{q})\}$ by the equation

$$
\tan \vartheta(x)=1 / \sinh (\ell(x) / 2), \quad \vartheta(x(\mathfrak{q}))=\pi / 2 .
$$

Denote by $\theta_{1}(x)(<\pi / 2)$ the outer angle between the line through two points $\zeta_{1}$ and $\zeta_{2}$ and the circle $\partial \Delta(H)$ at $\zeta_{1}$ (cf. the left picture of Figure 10). By definition, $\theta_{1}(x)<\theta(x)$ and hence there exists $l_{3}>0$ such that $\theta_{1}(x)<\vartheta(x)$ whenever $\ell(x)<l_{3}$. Notice that $\left|\zeta_{1}-\zeta_{2}\right|=2 \sinh (\ell(x) / 2) /\left|c_{B}\right|$ and the angle between $\sigma^{\prime}$ and 
$\sigma$ at $\zeta_{1}$ is equal to $\vartheta(x)$. Hence the angle between $\sigma^{\prime}$ and the line segment between $\zeta_{1}$ and $\zeta_{2}$ at $\zeta_{1}$ is equal to $\pi / 2+\vartheta(x)-\theta_{1}(x)$. Therefore, we obtain that

$$
\begin{aligned}
\operatorname{Area}\left(\mathcal{C}_{[B]}\right) & =\frac{\sinh ^{2}(\ell(x) / 2)}{2\left|c_{B}\right|^{2}}\left\{\frac{\pi+2\left(\vartheta(x)-\theta_{1}(x)\right)}{\cos ^{2}\left(\vartheta(x)-\theta_{1}(x)\right)}+2 \tan \left(\vartheta(x)-\theta_{1}(x)\right)\right\} \\
& \gtrsim\left|c_{B}\right|^{-2}\left\{\frac{\ell(x)}{\cos \left(\vartheta(x)-\theta_{1}(x)\right)}\right\}^{2},
\end{aligned}
$$

whenever $\ell(x)<l_{3}$, since $\vartheta(x)-\theta_{1}(x)>0$. Indeed, let $\zeta_{3}$ be the center of the circle containing $\sigma^{\prime}$. Then the set $\mathcal{C}_{[B]}$ is divided into two parts (cf. the right picture of Figure 10): the sector enclosed by $\sigma^{\prime}$ and two segments between $\zeta_{3}$ and $\zeta_{i}$ for $i=1,2$, and the triangle with vertices $\zeta_{1}, \zeta_{2}$, and $\zeta_{3}$. The first part in the bracket of the inequality above corresponds to the area of the sector, the second is the area of the triangle.

A simple calculation shows

$$
\begin{aligned}
\ell(x)^{-1} \cos \left(\vartheta(x)-\theta_{1}(x)\right) & =\ell(x)^{-1}\left(\cos \vartheta(x) \cos \theta_{1}(x)+\sin \vartheta(x) \sin \theta_{1}(x)\right) \\
& <\ell(x)^{-1} \cos \vartheta(x)+\ell(x)^{-1} \sin \theta(x) \\
& \lesssim \ell(x)^{-1} \tanh (\ell(x) / 2)+\ell(x)^{-1} \sin \ell(x) \\
& =O(1)
\end{aligned}
$$

by Lemma 7.1 where the last constant of the above inequality is dependent only on the implicit constant of the inequality in Lemma 7.1. Together with (7) we can find $l_{4}>0$, which is independent of the choice of the classes $[B] \in\left\langle X_{\mathfrak{q}, x}\right\rangle \backslash G_{x}$, such that if $\ell(x)<l_{4}$,

$$
\left|c_{B}\right|^{-2} \lesssim \operatorname{Area}\left(\mathcal{C}_{[B]}\right)
$$

where, by the argument above, the implicit constant depends only on the implicit constant of the inequality in Lemma 7.1 By applying Proposition 6.6 (Radius of peripheral disks), the assumption $F_{[B]} \cap\left\{|\zeta| \leq 2 \operatorname{Re} \lambda_{p / q}(x(\mathfrak{q}))\right\} \neq \emptyset$ implies that $\Delta(H)$ is contained in the disk of radius $2 \operatorname{Re} \lambda_{p / q}(x(\mathfrak{q}))+\delta_{0}$ with center 0 , where $\delta_{0}$ depends only on $\operatorname{Re} \lambda_{p / q}(x(\mathfrak{q}))$. Therefore we conclude that

$$
\sum_{[B]}^{\prime}\left|c_{B}\right|^{-2} \lesssim \sum_{[B]}^{\prime} \operatorname{Area}\left(\mathcal{C}_{[B]}\right) \leq \operatorname{Area}\left(\left\{|\zeta| \leq 2 \operatorname{Re} \lambda_{p / q}(x(\mathfrak{q}))+\delta_{0}\right\}\right)=O(1)
$$

as $\ell(x) \rightarrow 0$.

Finally, we should note that from the proof and Lemma 7.1 the last constant of the inequality above depends only on the translation length $\operatorname{Re} \lambda_{p / q}(x(\mathfrak{q}))$ of $X_{p / q, x(\mathfrak{q})}$, if $\mathcal{X}=\mathcal{M}$ or $\mathbf{w}(\mathfrak{q})$ does not correspond to the shortest geodesic on a hyperbolic surface $\left(\Sigma, \nu_{-}\left(\rho_{0}^{b}\right)\right), 0 \in \mathcal{B}_{G}$. In addition, it depends on the length of the shortest geodesic on $\left(\Sigma, \nu_{-}\left(\rho_{0}^{b}\right)\right)$.

\section{NON-VANISHING THEOREM FOR THE DERIVATIVE OF TRACE FUNCTIONS}

10.1. Non-vanishing theorem. We shall prove Theorem 2;

Theorem 2 (Rational boundary points are simple zeros). For all $\mathfrak{q} \in \mathbb{Q} \mathcal{X}$, it holds that

$$
\left.\frac{d}{d x} \operatorname{tr}^{2} X_{\mathfrak{q}, x}\right|_{x=x(\mathfrak{q})} \neq 0 .
$$

In particular, for all $\mathfrak{q} \in \mathbb{Q}_{\mathcal{X}}, x(\mathfrak{q})$ is a simple zero of the trace function $\operatorname{tr}^{2} X_{\mathfrak{q}, x}$. 
Remark 3. In the case of $\mathcal{X}=\mathcal{M}$, this theorem gives an affirmative answer to a conjecture of D. Wright 63].

10.2. Key lemma. We show the following:

Lemma 10.1. Suppose that $\mathcal{X}=\mathcal{M}$ or $\mathcal{B}_{G}$ for some quasifuchsian group $G$ of type $(1,1)$. Fix $\mathfrak{q} \in \mathbb{Q}_{\mathcal{X}}$. For $p / q \in \mathbb{Q}_{\mathcal{X}}$, we have

$$
\left.\left|\frac{d}{d x} \operatorname{tr}^{2} X_{p / q, x}\right|_{x=x(\mathfrak{q})}|\lesssim| \frac{d}{d x} \operatorname{tr}^{2} X_{\mathfrak{q}, x}\right|_{x=x(\mathfrak{q})} \mid .
$$

Moreover, the implicit constant is dependent only on the translation length of $X_{p / q, x}$ at $x=x(\mathfrak{q})$, either when $\mathcal{X}=\mathcal{M}$ or when $\mathcal{X}=\mathcal{B}_{G}$ and $\mathbf{w}(\mathfrak{q})$ does not correspond to the shortest geodesic on $\left(\Sigma, \nu_{-}\left(\rho_{0}^{b}\right)\right)$. Otherwise, it is also dependent on the length of the shortest geodesic on $\left(\Sigma, \nu_{-}\left(\rho_{0}^{b}\right)\right)$.

Proof. Let $p / q \in \mathbb{Q}_{\mathcal{X}}$ be as in the assumption. If $X_{p / q, x(\mathfrak{q})}$ is parabolic, $\mathcal{X}$ is the Maskit slice and $X_{p / q, x}$ is parabolic for all $x \in \mathcal{X}$ unless $p / q=\mathfrak{q}$. Therefore the derivative of $\operatorname{tr}^{2} X_{p / q, x}$ at $x=x(\mathfrak{q})$ vanishes, and hence the inequality above holds.

Assume that $X_{p / q, x(\mathfrak{q})}$ is loxodromic. We may assume that $p / q \neq \mathfrak{q}$. By virtue of Theorem 9 (Derivative is of linear order), we have

$$
\left|\frac{d}{d l}\left(\lambda_{p / q} \circ \ell^{-1}\right)(l)\right| \lesssim l \quad \text { as } l \rightarrow 0 .
$$

Let $x \in \mathcal{P}_{\mathfrak{q}}^{\mathcal{X}}$ with appropriately small $\ell(x)$. Integrating (8) from $l=0$ to $\ell(x)$, we obtain

$$
\left|\lambda_{p / q}(x)-\lambda_{p / q}(x(\mathfrak{q}))\right| \lesssim \ell(x)^{2} .
$$

Since $X_{\mathfrak{q}, x(\mathfrak{q})}$ is parabolic, the trace function of $X_{\mathfrak{q}, x}$ satisfies

$$
\left|\operatorname{tr}^{2} X_{\mathfrak{q}, x}-4\right| \asymp \ell(x)^{2}
$$

for $x \in \mathcal{P}_{\mathfrak{q}}^{\mathcal{X}}$ near $x(\mathfrak{q})$. Hence we have

$$
\left|\lambda_{p / q}(x)-\lambda_{p / q}(x(\mathfrak{q}))\right| \lesssim\left|\operatorname{tr}^{2} X_{\mathfrak{q}, x}-4\right|
$$

for $x \in \mathcal{P}_{\mathfrak{q}}^{\mathcal{X}}$ with $\ell(x) \rightarrow 0$. Since $\operatorname{tr}^{2} X_{p / q, x}=4 \cosh ^{2}\left(\lambda_{p / q}(x) / 2\right)$, dividing the inequality (9) by $|x-x(\mathfrak{q})|$ and letting $x \rightarrow x(\mathfrak{q})$ imply the inequality in the assertion.

The later part of this lemma follows from Theorem 9 .

10.3. Proof of Theorem 2, We shall show Theorem 2 2 in all slices.

10.3.1. The case $\mathcal{X}=\mathcal{M}$. In this case, $\mathcal{X}=\mathcal{M}, \mathcal{X}_{0}=\mathbb{C}, x(\mathfrak{q})=x^{m}(\mathfrak{q})$, and $\eta_{x}=\rho_{x}^{m}$. We shall show the following:

Proposition 10.1. There exists a universal constant $C_{M}>0$ such that

$$
\left|\frac{d}{d \mu} \operatorname{tr}^{2} \rho_{\mu}^{m}(\mathbf{w}(\mathfrak{q}))\right|_{\mu=x^{m}(\mathfrak{q})} \mid \geq C_{M}
$$

for all $\mathfrak{q} \in \mathbb{Q}$.

Proof. A simple calculation shows that $\operatorname{tr}^{2} \rho_{\mu}^{m}(\mathbf{w}(n / 1))=-(\mu-2 n)^{2}$, and hence

$$
\frac{d}{d \mu} \operatorname{tr}^{2} \rho_{\mu}^{m}(\mathbf{w}(n / 1))=-2(\mu-2 n)
$$


Let us consider annular domains

$$
\mathcal{A}_{n}:=\{|\operatorname{Re} \mu-2 n|<3,|\operatorname{Im} \mu-2|<3\}-\{|\operatorname{Re} \mu-2 n| \leq 1 / 3,|\operatorname{Im} \mu-2| \leq 2\}
$$

for all $n \in \mathbb{Z}$. Since $x^{m}(n / 1)=2 n+2 i, \rho_{\mu}^{m}(\mathbf{w}(n / 1))$ is loxodromic for $\mu \in \mathcal{A}_{n}$, its translation length of $\operatorname{tr}^{2} \rho_{\mu}^{m}(\mathbf{w}(n / 1))$ lies between $2 \sinh ^{-1}(1 / 6)$ and $2 \sinh ^{-1}(3 / 2)$, and the absolute value of the derivative of this trace function is greater than $2 / 3$. Furthermore, a collection $\left\{\mathcal{A}_{n}\right\}_{n}$ covers the strip $\{-1<\operatorname{Im} \mu<5\}$ and hence this collection covers the boundary $\partial \mathcal{M}$. Indeed, according to Proposition 2.6 of [24] (see also [33]), the boundary $\partial \mathcal{M}$ is contained in the strip $\{0<\operatorname{Im} \mu<2\}$. Hence, by Lemma 10.1, the absolute value of the derivative of $\operatorname{tr}^{2} \rho_{\mu}^{m}(\mathbf{w}(\mathfrak{q}))$ at $x=x^{m}(\mathfrak{q})$ is greater than a universal constant, say $C_{M}>0$.

10.3.2. The case $\mathcal{X}=\mathcal{E}$. In this case, $\mathcal{X}=\mathcal{E}, \mathcal{X}_{0}=\mathbb{C}^{*}, x(\mathfrak{q})=x^{e}(\mathfrak{q})$, and $\eta_{x}=\rho_{x}^{e}$. First, we show

Proposition 10.2. Let $p / q, p^{\prime} / q^{\prime} \in \hat{\mathbb{Q}}$ with $p / q \neq p^{\prime} / q^{\prime}$. Define a holomorphic function on the space of representations $\mathcal{R}$ by

$$
\Psi([\eta])=\left(\operatorname{tr}^{2} \eta(\mathbf{w}(p / q)), \operatorname{tr}^{2} \eta\left(\mathbf{w}\left(p^{\prime} / q^{\prime}\right)\right)\right) .
$$

Denote by $\left[\rho_{0}\right]$ the representation with the condition that its image $\rho_{0}\left(\pi_{1}\right)$ is a maximal parabolic group with parabolic transformations $\rho_{0}(\mathbf{w}(p / q)), \rho_{0}\left(\mathbf{w}\left(p^{\prime} / q^{\prime}\right)\right)$, and $\rho_{0}([\mathbf{a}, \mathbf{b}])$. Then the differential of $\Psi$ has maximal rank at $\left[\rho_{0}\right]$.

Proof. Since $\rho_{0}$ is irreducible, $\left[\rho_{0}\right]$ is a regular point of $\mathcal{R}$. We may assume that $\nu_{+}\left(\left[\rho_{0}\right]\right)=p / q$ and $\nu_{-}\left(\left[\rho_{0}\right]\right)=p^{\prime} / q^{\prime}$.

Let $\varsigma_{1}$ be an isomorphism of $\pi_{1}$ so that $\varsigma_{1}(\mathbf{a})=\mathbf{w}\left(p^{\prime} / q^{\prime}\right)$. Define $x_{1} \in \mathbb{Q}$ such that $\mathbf{w}\left(x_{1}\right)$ is conjugate to $\varsigma_{1}^{-1}(\mathbf{w}(p / q))$. By the injectivity of end invariants, $\left[\rho_{x^{m}\left(x_{1}\right)}^{m} \circ\right.$ $\left.\varsigma_{1}^{-1}\right]=\left[\rho_{0}\right]$ (see also [27]).

Let $\varsigma_{2}$ be an isomorphism of $\pi_{1}$ so that $\varsigma_{2}(\mathbf{a})=\mathbf{w}(p / q)$ and let $x_{2} \in \mathbb{Q}$ such that $\mathbf{w}\left(x_{2}\right)$ is conjugate to $\varsigma_{2}^{-1}\left(\mathbf{w}\left(p^{\prime} / q^{\prime}\right)\right)$. Put $\mathcal{I}(z)=\bar{z}$. Then it holds that

$$
\left[\mathcal{I} \rho \frac{m}{x^{m}\left(x_{2}\right)} \circ \varsigma_{2}^{-1} \mathcal{I}\right]:=\left[\pi_{1} \ni \mathbf{a}^{\prime} \mapsto \mathcal{I} \circ \rho \frac{m}{x^{m}\left(x_{2}\right)}\left(\varsigma_{2}^{-1}\left(\mathbf{a}^{\prime}\right)\right) \circ \mathcal{I}\right]=\left[\rho_{0}\right] .
$$

Let us consider the holomorphic mappings $\psi_{1}$ and $\psi_{2}$ of a sufficiently small disk $\{|t|<\epsilon\}$ to $\mathcal{R}$ defined by

$$
\begin{aligned}
& \psi_{1}(t)=\left[\rho_{x^{m}\left(x_{1}\right)+t}^{m} \circ \varsigma_{1}^{-1}\right], \\
& \psi_{2}(t)=\left[\mathcal{I} \rho \frac{m}{x^{m}\left(x_{2}\right)}+\bar{t} \circ \varsigma_{2}^{-1} \mathcal{I}\right] .
\end{aligned}
$$

By definition, this satisfies that $\psi_{k}(0)=\left[\rho_{0}\right]$ for $k=1,2$ and

$$
\begin{aligned}
& \Psi \circ \psi_{1}(t)=\left(4, \operatorname{tr}^{2} \rho_{\left(x^{m}\left(x_{1}\right)+t\right)}^{m}\left(\mathbf{w}\left(x_{1}\right)\right)\right), \\
& \Psi \circ \psi_{2}(t)=\left(\overline{\operatorname{tr}^{2} \rho \frac{m}{x^{m}\left(x_{2}\right)}+\bar{t}}\left(\mathbf{w}\left(x_{2}\right)\right), 4\right)=\left(\operatorname{tr}^{2} \rho_{x^{m}\left(x_{2}\right)+t}^{m}\left(\mathbf{w}\left(x_{2}\right)\right), 4\right),
\end{aligned}
$$

since $\operatorname{tr}^{2} \rho_{\mu}^{m}\left(\mathbf{w}\left(x_{2}\right)\right)$ is a polynomial of real coefficients on the $\mu$-plane. Together with Theorem 2 for $\mathcal{X}=\mathcal{M}$, we complete the proof of Proposition 10.2

Proof of Theorem 2 . Define the mapping $\psi$ from $\mathbb{C}$ to $\mathcal{R}$ by $\psi(d)=\left[\rho_{d}^{e}\right]$. Then

$$
\left.\frac{d}{d c} \operatorname{tr}^{2} \psi(c)\left(\mathbf{a b}^{-1}\right)\right|_{c=x^{e}(\mathfrak{q})}=\left.\frac{d}{d c} \operatorname{tr}^{2} \rho_{c}^{e}\left(\mathbf{a b}^{-1}\right)\right|_{c=x^{e}(\mathfrak{q})}=8 x^{e}(\mathfrak{q})\left(4 x^{e}(\mathfrak{q})^{2}+2\right) .
$$

Since $\rho_{x^{e}(\mathfrak{q})}^{e}$ is faithful and $\operatorname{tr}^{2} \psi\left(x^{e}(\mathfrak{q})\right)\left(\mathbf{a b}^{-1}\right)=\left(4 x^{e}(\mathfrak{q})^{2}+2\right)^{2}, 4 x^{e}(\mathfrak{q})^{2}+2 \neq 0$. At $c=0$, the representation $\rho_{c}^{e}$ is divergent. Hence it is impossible that $x^{e}(\mathfrak{q})=0$. 
Therefore, the differential of $\psi$ has the maximal rank at $d=x^{e}(\mathfrak{q})$. Define a function by

$$
\Psi([\eta])=\left(\operatorname{tr}^{2} \eta(\mathbf{w}(\mathfrak{q})), \operatorname{tr}^{2} \eta\left(\mathbf{w}\left(\mathfrak{q}^{-1}\right)\right)\right) .
$$

The condition $\mathfrak{q} \neq \pm 1 / 1$ implies $\mathfrak{q} \neq \mathfrak{q}^{-1}$. Hence from Proposition 10.2, the differential of $\Psi$ has the maximal rank at $\left[\rho_{x}^{e}(\mathfrak{q})\right]$. Since $\operatorname{tr}^{2} \rho_{d}^{e}(\mathbf{w}(\mathfrak{q}))=\operatorname{tr}^{2} \rho_{d}^{e}\left(\mathbf{w}\left(\mathfrak{q}^{-1}\right)\right)$ for $d \in \mathbb{C}^{*}$, and

$$
\Psi \circ \psi(d)=\left(\operatorname{tr}^{2} \rho_{d}^{e}(\mathbf{w}(\mathfrak{q})), \operatorname{tr}^{2} \rho_{d}^{e}\left(\mathbf{w}\left(\mathfrak{q}^{-1}\right)\right)\right),
$$

we conclude the desired statement.

10.3.3. The case $\mathcal{X}=\mathcal{B}_{G}$. In this case, there exists a quasifuchsian group $G$ of type $(1,1)$ such that $\mathcal{X}=\mathcal{B}_{G}, \mathcal{X}_{0}=Q_{-4}(G), x(\mathfrak{q})=x^{b}(\mathfrak{q})$, and $\eta_{x}=\rho_{x}^{b}$. To prove Theorem 2 in this case, we will show

Proposition 10.3. Let $G=G\left(z_{0}, y_{0}\right)$ and $y_{0}=\left(g_{0}, S_{0}\right)$. Then there exists a constant $C_{S_{0}}>0$ dependent only on a Riemann surface $S_{0}$ such that

$$
\left|\frac{d}{d \varphi} \operatorname{tr}^{2} \rho_{\varphi}^{b}(\mathbf{w}(\mathfrak{q}))\right|_{\varphi=x^{b}(\mathfrak{q})} \mid \geq C_{S_{0}}
$$

for all $\mathfrak{q} \in \hat{\mathbb{Q}}$.

The proof of this proposition needs several propositions. Henceforth, we fix two points $z_{0}=\left(f_{0}, R_{0}\right) \in \operatorname{Teich}(\Sigma)$ and $y_{0}=\left(g_{0}, S_{0}\right) \in \operatorname{Teich}(\bar{\Sigma})$ as in Section 2

Trace functions on the boundary of a Bers slice. We first state the following proposition.

Proposition 10.4. Let $G=G\left(z_{0}, y_{0}\right)$. For any $\varphi_{0} \in \partial \mathcal{B}_{G}$, there exists $\mathfrak{p} \in \hat{\mathbb{Q}}$ such that $\rho_{\varphi_{0}}^{b}(\mathbf{w}(\mathfrak{p}))$ is loxodromic and that the derivative of the trace function

$$
Q_{-4}\left(z_{0}, y_{0}\right) \ni \varphi \mapsto \operatorname{tr}^{2} \rho_{\varphi}^{b}(\mathbf{w}(\mathfrak{p}))
$$

at $\varphi=\varphi_{0}$ does not vanish.

To show this proposition, we first note the facts that from the normalization of $W_{y}^{x}$ (see $\$ 2.4$ ) , the set

$$
\mathcal{T}^{*}:=\left\{(y, \varphi) \mid y \in \operatorname{Teich}(\bar{\Sigma}), \varphi \in Q\left(z_{0}, y\right)\right\}
$$

is recognized as the cotangent space on Teich $(\bar{\Sigma})$ (cf. [8]), and by a theorem of Hejhal in [20] (see also [4]]), the holonomy mapping

$$
\mathcal{T}^{*} \ni(y, \varphi) \mapsto\left[\rho_{\varphi}^{b}\right] \in \mathcal{R}
$$

is an analytic local homeomorphism.

Next let us recall the Jørgensen parameter of once punctured torus groups: Let $a, b, c \in \mathbb{C}$ with $c \neq 0$ and $a^{2}+b^{2}+c^{2}=a b c$. Then we construct an admissible homomorphism (cf. Section 2) $\eta_{(a, b, c)}$ by

$$
\eta_{(a, b, c)}(\mathbf{a}):=\left[\begin{array}{cc}
a-b / c & a / c^{2} \\
a & b / c
\end{array}\right], \quad \eta_{(a, b, c)}(\mathbf{b}):=\left[\begin{array}{cc}
b-a / c & -b / c^{2} \\
-b & a / c
\end{array}\right] .
$$

Notice that

$$
\operatorname{tr}^{2} \eta_{(a, b, c)}(\mathbf{a})=a^{2}, \quad \operatorname{tr}^{2} \eta_{(a, b, c)}(\mathbf{b})=b^{2}, \quad \text { and } \quad \operatorname{tr}^{2} \eta_{(a, b, c)}(\mathbf{a b})=c^{2} .
$$


We also note that a point $(a, b, c)$ in the 2 -dimensional affine variety $\mathcal{V}:=\left\{a^{2}+\right.$ $\left.b^{2}+c^{2}=a b c\right\}$ is a regular point if $a b c \neq 0$. We first note the following well-known lemma.

Lemma 10.2. For $[\eta] \in \mathcal{D}$, there exists a regular point $(a, b, c) \in \mathcal{V}$ such that $[\eta]=\left[\eta_{(a, b, c)}\right]$.

The argument of this proof is due to T. Jørgensen [23]. We sketch his proof here.

Proof. Let $\widetilde{\eta(\mathbf{a})}$ and $\widetilde{\eta(\mathbf{b})}$ be lifts $\eta(\mathbf{a})$ and $\eta(\mathbf{b})$ on $\mathrm{SL}_{2}(\mathbb{C})$, respectively. Since $\rho$ is a discrete representation, the trace of the commutator of these two matrices is equal to -2 (cf. e.g. Theorem 5.37 of [40]). Since any two $A, B \in \mathrm{SL}_{2}(\mathbb{C})$ satisfy the identity

$$
\operatorname{tr}^{2} A+\operatorname{tr}^{2} B+\operatorname{tr}^{2} A B=\operatorname{tr} A \operatorname{tr} B \operatorname{tr} A B+2+\operatorname{tr}\left(A B A^{-1} B^{-1}\right),
$$

the traces $a:=\operatorname{tr} \widetilde{\eta(\mathbf{a})}, b:=\operatorname{tr} \widetilde{\eta(\mathbf{b})}$, and $c:=\operatorname{tr}(\widetilde{\eta(\mathbf{a})} \widetilde{\eta(\mathbf{b})})$ satisfies the equation $a^{2}+b^{2}+c^{2}=a b c$. Furthermore, all of these are not elliptic order 2; we have $a b c \neq 0$. This means that $(a, b, c) \in \mathcal{V}$ and this point is a regular point.

We take a conjugation so that $\eta([\mathbf{a}, \mathbf{b}])(z)=z+2$ and, after this, we take a conjugation again by the translation which takes the pole $(A B)^{-1}(\infty)$ of $A B$ to zero. Then we can observe that $[\eta]=\left[\eta_{(a, b, c)}\right]$. Here the pole of a matrix

$$
\left(\begin{array}{ll}
a & b \\
c & d
\end{array}\right)
$$

is $a / c$.

Hence we have the following proposition.

Proposition 10.5. Let $\omega$ be an orientation preserving homeomorphism on $\Sigma$. Then the holomorphic mapping

$$
\mathcal{V} \ni(a, b, c) \mapsto\left[\eta_{(a, b, c)} \circ\left(\omega_{*}\right)^{-1}\right] \in \mathcal{R}
$$

admits a local inverse mapping at a point $(a, b, c)$ which satisfies $\left[\eta_{(a, b, c)}\right] \in \mathcal{D}$.

Remark 4. By definition, a local inverse mapping forms

$$
[\eta] \mapsto\left(\operatorname{tr} \eta \circ \omega_{*}(\mathbf{a}), \operatorname{tr} \eta \circ \omega_{*}(\mathbf{b}), \operatorname{tr} \eta \circ \omega_{*}(\mathbf{a b})\right) \in \mathcal{V} .
$$

Proof. It is easy to see that the mapping

$$
\mathcal{V} \ni(a, b, c) \mapsto\left[\eta_{(a, b, c)}\right] \in \mathcal{R}
$$

is injective and holomorphic. By Lemma 10.2, a set $\mathcal{D}$ is contained in the image of the mapping above. Since $[\eta] \in \mathcal{D}$ and its corresponding point in $\mathcal{V}$ are both regular points of the ambient manifolds, the mapping above admits local inverse mapping at a neighborhood of $[\eta]$. The mappings discussed here are represented by the inverse direction of the broken lines (see Figure 11).

Since the mapping $\mathcal{R} \ni[\eta] \mapsto\left[\eta \circ\left(\omega_{*}\right)^{-1}\right] \in \mathcal{R}$ is a holomorphic automorphism of $\mathcal{R}$ (cf. [43]), we conclude the assertion.

Proof of Proposition 10.4. Recall that $G=G\left(z_{0}, y_{0}\right)$. Let $\varphi_{0} \in \partial \mathcal{B}_{G}$. By definition, we can recognize $\varphi_{0}$ as a point $\left(y_{0}, \varphi_{0}\right) \in \mathcal{T}^{*}$. Since the holonomy mapping

$$
\mathcal{T}^{*} \ni(y, \varphi) \mapsto\left[\rho_{\varphi}^{b}\right] \in \mathcal{R}
$$




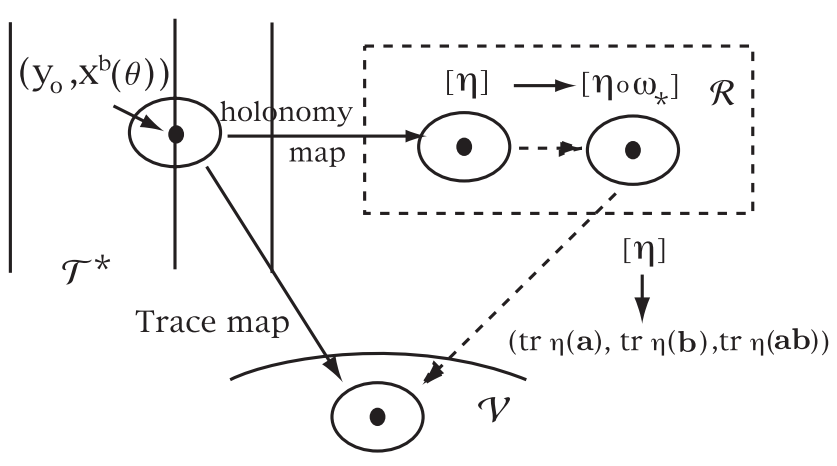

Figure 11. Trace map is locally biholomorphic

is an analytic local homeomorphism, we can take a neighborhood $\mathcal{U}$ of $\left(y_{0}, \rho_{\varphi_{0}}^{b}\right) \in$ $\mathcal{T}^{*}$, at which the holonomy mapping is homeomorphic.

Let $\omega$ be an orientation preserving homeomorphism of $\Sigma$ so that all $\rho_{\varphi_{0}}^{b} \circ \omega_{*}(\mathbf{a})$, $\rho_{\varphi_{0}}^{b} \circ \omega_{*}(\mathbf{b})$, and $\rho_{\varphi_{0}}^{b} \circ \omega_{*}(\mathbf{a b})$ are loxodromic at $\varphi=\varphi_{0}$. Such $\omega$ does exist, because $\rho_{\varphi_{0}}^{b}$ can admit at most two accidental parabolic transformations.

By composing the local inverse mapping defined in Proposition 10.5 and the homeomorphism defined by the inverse of the holonomy mapping (cf. Figure 11), we conclude that the trace map

$$
\mathcal{U} \ni(y, \varphi) \mapsto\left(\operatorname{tr} \rho_{\varphi}^{b} \circ \omega_{*}(\mathbf{a}), \operatorname{tr} \rho_{\varphi}^{b} \circ \omega_{*}(\mathbf{b}), \operatorname{tr} \rho_{\varphi}^{b} \circ \omega_{*}(\mathbf{a b})\right) \in \mathcal{V}
$$

is well-defined and biholomorphic. This means that, on the differential of the trace map along a fiber of $\mathcal{T}^{*}$ passing through $\left(y_{0}, \varphi_{0}\right)$, one of their coordinates of the right-hand side does not vanish. This implies the assertion.

Bers' marking trick. Here we shall give notation and properties for the Bers' marking trick. The proposition proved here is also used in the next section for proving the quasiconformal extendibility of Teichmüller modular transformations. For the proof of Proposition 10.3, we need to distinguish where the discussing differential lies. Hence, for $(z, y) \in \operatorname{Teich}(\Sigma) \times \operatorname{Teich}(\bar{\Sigma})$ and $\varphi \in Q_{-4}(z, y)$, we denote by $\rho_{\varphi,(z, y)}^{b}$ the holonomy of the Schwarzian equation with respect to $\varphi$ instead of $\rho_{\varphi}^{b}$ (see Section [2.4).

Definition 13 (Affine mapping $\left.L_{y}^{x, z}\right)$. Let $z^{\prime}, z \in \operatorname{Teich}(\Sigma)$ and $y \in \operatorname{Teich}(\bar{\Sigma})$. The affine mapping $L_{y}^{z^{\prime}, z}$ of $Q_{-4}(z, y)$ to $Q_{-4}\left(z^{\prime}, y\right)$ is defined by the equation

$$
L_{y}^{z^{\prime}, z}(\varphi)=\varphi \circ\left(f_{y}^{z, z^{\prime}}\right) \times\left(f_{y}^{z, z^{\prime}}\right)^{\prime 2}+\mathcal{S}\left(f_{y}^{z, z^{\prime}}\right) .
$$

Recall that for $\left(z^{\prime}, y\right) \in \operatorname{Teich}(\Sigma) \times \operatorname{Teich}(\bar{\Sigma}), W_{y}^{z^{\prime}}$ is a quasiconformal mapping on $\hat{\mathbb{C}}$ such that $W_{y}^{z^{\prime}}$ fixes 0,1 , and $\infty$, and that a representation

$$
\pi_{1} \ni \mathbf{a}^{\prime} \mapsto W_{y}^{z^{\prime}} \circ \eta_{G\left(z_{0}, y_{0}\right)}\left(\mathbf{a}^{\prime}\right) \circ\left(W_{y}^{z^{\prime}}\right)^{-1} \in \mathrm{PSL}_{2}(\mathbb{C})
$$

is conjugate to $\eta_{G\left(z^{\prime}, y\right)}$ (see Section [2.4). 
Proposition 10.6. The following two statements hold:

(1) For all $z \in \operatorname{Teich}(\Sigma)$ and $\varphi \in Q_{-4}\left(z, y_{0}\right)$,

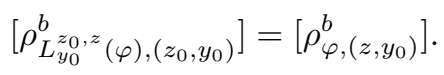

(2) For $[\omega] \in \operatorname{Mod}(\Sigma)$, there is a Möbius transformation $P$ such that

$$
\Omega_{-}\left(\left[\omega_{*}\right]\left(z_{0}\right),\left[\bar{\omega}_{*}\right]\left(y_{0}\right)\right)=P\left(\Omega_{-}\left(z_{0}, y_{0}\right)\right)
$$

and that a linear mapping $\mathcal{L}_{P}$ from $Q_{-4}\left([\omega]_{*}\left(z_{0}\right),[\bar{\omega}]_{*}\left(y_{0}\right)\right)$ to $Q_{-4}\left(z_{0}, y_{0}\right)$ by $\mathcal{L}_{P}(\varphi)=\varphi \circ P\left(P^{\prime}\right)^{2}$ satisfies the formula:

$$
\left[\rho_{\mathcal{L}_{P}(\varphi),\left(z_{0}, y_{0}\right)}^{b} \circ\left(\omega_{*}\right)^{-1}\right]=\left[\rho_{\varphi,\left(\left[\omega_{*}\right]\left(z_{0}\right),\left[\omega_{*}\right]\left(y_{0}\right)\right)}^{b}\right] .
$$

Proof. (1) Let $\varphi \in Q_{-4}\left(z, y_{0}\right)$ and $h_{0}$ be the local univalent function with $\mathcal{S}\left(h_{0}\right)=$ $\varphi$. According to the well-known transformation (cf. [36]) on the Schwarzian derivative,

$$
\mathcal{S}\left(h_{0} \circ f_{y_{0}}^{z_{0}, z}\right)=L_{y_{0}}^{z_{0}, z}(\varphi) \in Q_{-4}\left(z, y_{0}\right) .
$$

This means that the holonomies for $\varphi$ and $L_{y_{0}}^{z_{0}, z}(\varphi) \in Q_{-4}\left(z, y_{0}\right)$ satisfy the equation in (1).

(2) Let $z_{0}^{\prime}=\left[\omega_{*}\right]\left(z_{0}\right)$ and $y_{0}^{\prime}=\left[\bar{\omega}_{*}\right]\left(y_{0}\right)$. Then by virtue of Bers' simultaneous uniformization (cf. Section 8 of [8]. See also p.46, Section 3.3 of [43]), we have that

$$
\begin{aligned}
{\left[\eta_{G\left(z_{0}^{\prime}, y_{0}^{\prime}\right)}\right] } & =\left[\eta_{G\left(\left(f_{0} \circ \omega^{-1}, R_{0}\right),\left(g_{0} \circ \bar{\omega}^{-1}, S_{0}\right)\right)}\right] \\
& =\left[\eta_{G\left(\left(f_{0}, R_{0}\right),\left(g_{0}, S_{0}\right)\right)} \circ\left(\omega_{*}\right)^{-1}\right] \\
& =\left[\eta_{G\left(z_{0}, y_{0}\right)} \circ\left(\omega_{*}\right)^{-1}\right] .
\end{aligned}
$$

Hence there exists a Möbius transformation $P$ so that

$$
P^{-1} \circ \eta_{G\left(z_{0}^{\prime}, y_{0}^{\prime}\right)}(\mathbf{w}) \circ P=\eta_{G\left(z_{0}, y_{0}\right)} \circ\left(\omega_{*}\right)^{-1}(\mathbf{w})
$$

for all $\mathbf{w} \in \pi_{1}$. This implies that $\Omega_{-}\left(z_{0}^{\prime}, y_{0}^{\prime}\right)=P\left(\Omega_{-}\left(z_{0}, y_{0}\right)\right)$. Hence we can define a linear mapping $\mathcal{L}_{P}$ from $\varphi \in Q_{-4}\left(z_{0}^{\prime}, y_{0}^{\prime}\right)$ to $Q_{-4}\left(z_{0}, y_{0}\right)$ by $\mathcal{L}_{P}(\varphi)=$ $\varphi \circ P \cdot\left(P^{\prime}\right)^{2}$. By definition, for $\varphi \in Q_{-4}\left(z_{0}^{\prime}, y_{0}^{\prime}\right)$, the solution of the Schwarzian equation $\mathcal{S}(-)=\mathcal{L}_{P}(\varphi)$ on $\Omega_{-}\left(z_{0}, y_{0}\right)$ is equal to the precomposition of $P$ to the solution of the equation $\mathcal{S}(-)=\varphi$ on $\Omega_{-}\left(z_{0}^{\prime}, y_{0}^{\prime}\right)$, up to composing a Möbius transformation. Together with the equation (10), we have the assertion.

Proof of Proposition 10.3. Let us show Proposition 10.3. For any $\varphi \in \partial \mathcal{B}_{G} \subset$ $Q_{-4}\left(z_{0}, y_{0}\right)$, we can find $\mathfrak{p}=\mathfrak{p}_{\varphi} \in \hat{\mathbb{Q}}$ with the conditions in Proposition 10.4 Hence there exists a neighborhood $\mathcal{U}_{\varphi}^{\prime}$ of $\varphi$ such that for $\varphi^{\prime} \in \mathcal{U}_{\varphi}^{\prime}, \rho_{\varphi^{\prime},\left(z_{0}, y_{0}\right)}^{b}(\mathbf{w}(\mathfrak{p}))$ is loxodromic and the derivative of $\operatorname{tr}^{2} \rho_{\varphi,\left(z_{0}, y_{0}\right)}^{b}(\mathbf{w}(\mathfrak{p}))$ at $\varphi=\varphi^{\prime}$ does not vanish. Let $\mathcal{U}_{\varphi}$ be a neighborhood of $\varphi$ whose closure is compact in $\mathcal{U}_{\varphi}^{\prime}$. Then the collection $\left\{\mathcal{U}_{\varphi}\right\}, \varphi \in \partial \mathcal{B}_{G}$ forms a covering of the compact set $\partial \mathcal{B}_{G}$, and hence there exists a finite collection $\left\{\mathcal{U}_{\varphi_{i}}\right\}_{i}$ which covers $\partial \mathcal{B}_{G}$.

Let $\mathfrak{q} \in \hat{\mathbb{Q}}$. Take $i$ with $x^{b}(\mathfrak{q}) \in \mathcal{U}_{\varphi_{i}}$. Then by Lemma 10.1, the absolute value of the derivative of $\operatorname{tr}^{2} \rho_{\varphi,\left(z_{0}, y_{0}\right)}^{b}(\mathbf{w}(\mathfrak{q}))$ at $\varphi=x^{b}(\mathfrak{q})$ is greater than $C_{G, i}>0$, which depends on the translation length of $\rho_{\varphi_{i},\left(z_{0}, y_{0}\right)}^{b}\left(\mathbf{w}\left(\mathfrak{p}_{\varphi_{i}}\right)\right)$, the absolute value of the derivative of $\operatorname{tr}^{2} \rho_{\varphi,\left(z_{0}, y_{0}\right)}^{b}\left(\mathbf{w}\left(\mathfrak{p}_{\varphi_{i}}\right)\right)$ on $\varphi \in \mathcal{U}_{\varphi_{i}} \cap \partial \mathcal{B}_{G}$, and the complex structure of 
the negative end invariant of $G$. Thus the constant $C_{G}:=\min _{i}\left\{C_{G, i}\right\}$ satisfies that

$$
\left|\frac{d}{d \varphi} \operatorname{tr}^{2} \rho_{\varphi,\left(z_{0}, y_{0}\right)}^{b}(\mathbf{w}(\mathfrak{q}))\right|_{\varphi=x^{b}(\mathfrak{q})} \mid \geq C_{G}
$$

for all $\mathfrak{q} \in \hat{\mathbb{Q}}$.

Henceforth, we show that the constant $C_{G}$ is dependent only on a Riemann surface $S_{0}$, which is the underlying Riemann surface of the Teichmüller parameter $y_{0}$ for the negative end of $G$.

By definition, an affine mapping $L_{y_{0}}^{z_{0}, z}$ satisfies $\left\|L_{y_{0}}^{z_{0}, z}(\varphi)-L_{y_{0}}^{z_{0}, z}\left(\varphi^{\prime}\right)\right\|=\left\|\varphi-\varphi^{\prime}\right\|$. Hence by (1) of Proposition 10.6, we have

$$
\left|\frac{d}{d \varphi} \operatorname{tr}^{2} \rho_{\varphi,\left(z, y_{0}\right)}^{b}(\mathbf{w}(\mathfrak{q}))\right|=\left|\frac{d}{d \varphi} \operatorname{tr}^{2} \rho_{L_{y_{0}}^{z_{0}, z}(\varphi),\left(z_{0}, y_{0}\right)}^{b}(\mathbf{w}(\mathfrak{q}))\right|
$$

for $\varphi \in Q_{-4}\left(z, y_{0}\right)$. This means the lower bound of the absolute values of the derivative of trace functions is independent of the choice of the Teichmüller parameter of $G=G\left(z_{0}, y_{0}\right)$ of the positive end.

Let $\left[\bar{\omega}_{*}\right] \in \operatorname{Mod}(\bar{\Sigma})$ (see Section [2.2). Then by (2) of Proposition 10.6 there exists a Möbius transformation $P$ such that $\mathcal{L}_{P}(\varphi)=\varphi \circ P \cdot\left(P^{\prime}\right)^{2}$ defines a linear mapping from $Q_{-4}\left(\left[\omega_{*}\right]\left(z_{0}\right),\left[\bar{\omega}_{*}\right]\left(y_{0}\right)\right)$ to $Q_{-4}\left(z_{0}, y_{0}\right)$ satisfying

$$
\left[\rho_{\mathcal{L}_{P}(\varphi),\left(z_{0}, y_{0}\right)}^{b} \circ\left(\omega_{*}\right)^{-1}\right]=\left[\rho_{\varphi,\left(\left[\omega_{*}\right]\left(z_{0}\right),\left[\bar{\omega}_{*}\right]\left(y_{0}\right)\right)}^{b}\right]
$$

for all $\varphi \in Q_{-4}\left(z_{0}, y_{0}\right)$. Since $\mathcal{L}_{P}$ is an isometry, we have

$$
\left|\frac{d}{d \varphi} \rho_{\mathcal{L}_{P}(\varphi),\left(z_{0}, y_{0}\right)}^{b} \circ\left(\omega_{*}\right)^{-1}(\mathbf{w}(\mathfrak{q}))\right|=\left|\frac{d}{d \varphi} \rho_{\varphi,\left(\left[\omega_{*}\right]\left(z_{0}\right),\left[\bar{\omega}_{*}\right]\left(y_{0}\right)\right)}^{b}(\mathbf{w}(\mathfrak{q}))\right|
$$

for $\varphi \in Q_{-4}\left(\left[\omega_{*}\right]\left(z_{0}\right),\left[\bar{\omega}_{*}\right]\left(y_{0}\right)\right)$. This implies that the lower bound of the derivatives is independent of the choice of the marking of $y_{0}$.

\section{Teichmüller modular group ACting ON Bers SLICES}

In this section, we shall prove the quasiconformal extendibility of Teichmüller modular transformation acting on one-dimensional Teichmüller space. We frequently use the notation and symbols defined in Section 2.4 for Bers slices.

Remark 5. L. Bers [7] had proved that every Teichmüller modular transformation acting on $\mathcal{B}_{G}$ is extended continuously on its closure. 3

11.1. Improved $\lambda$-lemma. For more details, the reader can consult papers 39, 9], [14], [60], [58], or 61].

Definition 14 (Labeled holomorphic motions [41]). A labeled holomorphic motion of a set $A$ (in $\hat{\mathbb{C}}$ ) over a complex manifold $M$ with distinguished point $m \in M$ is a map $f: M \times A \rightarrow \hat{\mathbb{C}}$ such that:

(i) for any fixed $a \in A, f(\lambda, a)$ is a holomorphic function of $\lambda \in M$;

(ii) for any fixed $\lambda \in M, f(\lambda, a)$ is an injective function of $a \in A$; and

(iii) $f(m, a)=a$ for all $a \in A$.

\footnotetext{
${ }^{3}$ Here we consider the one-dimensional case. Compare with [28].
} 
Theorem 15 (Improved $\lambda$-lemma). Suppose that $M$ is conformally equivalent to the unit disk in $\mathbb{C}$. Then every holomorphic motion $f$ of a set $A$ over $M$ with distinguished point $m \in M$ can be extended as that of $\hat{\mathbb{C}}$ over $M$ with the following property: For all $\lambda \in M, f(\lambda, \cdot)$ is a quasiconformal mapping of the Riemann sphere onto itself with dilatation $\exp \left(d_{M}(m, \lambda)\right)$, where $d_{M}$ is the hyperbolic distance on $M$ with curvature -1 .

A proposition. We shall see the Bers' marking trick with respect to the Bers embeddings. The argument of the proof for this is similar to that of Proposition 10.6 Recall that $L_{y_{0}}^{z_{0}, z}$ is a linear mapping from $Q_{-4}\left(z, y_{0}\right)$ to $Q_{-4}\left(z_{0}, y_{0}\right)$ which is defined by the pull-back via the univalent function $f_{y_{0}}^{z, z_{0}}$ from $\Omega_{-}\left(z_{0}, y_{0}\right)$ to $\Omega_{-}\left(z, y_{0}\right)$ (see Definition 13).

Proposition 11.1. The following three statements hold:

(1) $L_{y_{0}}^{z_{0}, z} \circ \beta_{y_{0}}^{z}=\beta_{y_{0}}^{z_{0}}$ for all $z \in \operatorname{Teich}(\Sigma)$.

(2) For $[\omega] \in \operatorname{Mod}(\Sigma)$, there is a Möbius transformation $P$ such that

$$
G\left(z_{0},[\bar{\omega}]_{*}^{-1}\left(y_{0}\right)\right)=P G\left([\omega]_{*}\left(z_{0}\right), y_{0}\right) P^{-1}
$$

as Kleinian groups (i.e. both sides coincide without marking) and

$$
\Omega_{-}\left(z_{0},[\bar{\omega}]_{*}^{-1}\left(y_{0}\right)\right)=P\left(\Omega_{-}\left([\omega]_{*}\left(z_{0}\right), y_{0}\right)\right) .
$$

(3) Let $P$ be as above. Define a linear mapping $\mathcal{L}_{P}$ from $Q_{-4}\left(z_{0},[\bar{\omega}]_{*}^{-1}\left(y_{0}\right)\right)$ to $Q_{-4}\left([\omega]_{*}\left(z_{0}\right), y_{0}\right)$ by $\mathcal{L}_{P}(\varphi)=\varphi \circ P\left(P^{\prime}\right)^{2}$. Then

$$
\beta_{y_{0}}^{[\omega]_{*}\left(z_{0}\right)} \circ[\omega]_{*}(z)=\mathcal{L}_{P} \circ \beta_{[\bar{\omega}]_{*}^{-1}\left(y_{0}\right)}^{z_{0}}(z), \text { for } z \in \operatorname{Teich}(\Sigma) .
$$

Proof. The equation in (1) follows from the well-known transformation on the Schwarzian derivative as in (1) of Proposition 10.6

The second and third statements are obtained by the normalization of $W_{y}^{z^{\prime}}$ and by the Bers marking trick as in the previous section. Indeed, denote by $q_{w}$ a quasiconformal mapping on $\hat{\mathbb{C}}$ so that $q_{w}\left(\Omega_{-}\left(z_{0}, y_{0}\right)\right)=\Omega_{-}\left(z_{0}, y_{0}\right)$ and $q_{w}$ corresponds to the lift of $f_{0} \circ \omega^{-1} \circ f_{0}^{-1}$ on $\Omega_{+}\left(z_{0}, y_{0}\right)$ and the lift of $g_{0} \circ \bar{\omega}^{-1} \circ g_{0}^{-1}$ on $\Omega_{-}\left(z_{0}, y_{0}\right)$. Then $q_{\omega} G\left(z_{0}, y_{0}\right) q_{\omega}^{-1}=G\left(z_{0}, y_{0}\right)$ as Kleinian groups. Notice that this conjugation corresponds to the action $[\eta] \mapsto\left[\eta \circ\left(\omega_{*}\right)^{-1}\right]$ on $\mathcal{R}$.

Denote by $P$ the Möbius transformation sending three points 0,1 , and $\infty$ to $W_{[\bar{\omega}]_{*}^{-1}\left(y_{0}\right)}^{z_{0}}\left(q_{\omega}(0)\right), W_{[\bar{\omega}]_{*}^{-1}\left(y_{0}\right)}^{z_{0}}\left(q_{\omega}(1)\right)$, and $W_{[\bar{\omega}]_{*}^{-1}\left(y_{0}\right)}^{z_{0}}\left(q_{\omega}(\infty)\right)$, respectively. Notice that two quasiconformal mappings $P^{-1} \circ W_{[\bar{\omega}]_{*}^{-1}\left(y_{0}\right)}^{z_{0}}$ and $W_{y_{0}}^{[\omega]_{*}\left(z_{0}\right)} \circ q_{\omega}^{-1}$ uniformize two markings $f_{0} \circ \omega^{-1}: \Sigma \rightarrow R_{0}$ and $g_{0} \circ \omega: \bar{\Sigma}^{-1} \rightarrow S_{0}$, and take same values on three points $\left\{q_{\omega}(0), q_{\omega}(1), q_{\omega}(\infty)\right\}$. Hence we have

$$
P^{-1} \circ W_{[\bar{\omega}]_{*}^{-1}\left(y_{0}\right)}^{z_{0}}=W_{y_{0}}^{[\omega]_{*}\left(z_{0}\right)} \circ q_{\omega}^{-1},
$$

on the limit set of $G\left(z_{0}, y_{0}\right)$, because the value of such a quasiconformal mapping on the limit set is determined by the corresponding markings. Thus we obtain that

$$
G\left(z_{0},[\bar{\omega}]_{*}^{-1}\left(y_{0}\right)\right)=P G\left([\omega]_{*}\left(z_{0}\right), y_{0}\right) P^{-1}
$$

as Kleinian groups and $\Omega_{-}\left(z_{0},[\bar{\omega}]_{*}^{-1}\left(y_{0}\right)\right)=P\left(\Omega_{-}\left([\omega]_{*}\left(z_{0}\right), y_{0}\right)\right)$.

Suppose $z \in \operatorname{Teich}(\Sigma)$. Then by the same argument as above, there exists a Möbius transformation $P_{z}$ so that $P_{z}^{-1} \circ W_{[\bar{\omega}]_{*}^{-1}\left(y_{0}\right)}^{z}=W_{y_{0}}^{[\omega]_{*}(z)} \circ q_{\omega}^{-1}$. Thus we 
conclude that

$$
\begin{aligned}
\beta_{y_{0}}^{[\omega]_{*}\left(z_{0}\right)} \circ[\omega]_{*}(z) & =\mathcal{S}\left(f_{y_{0}}^{[\omega]_{*}(z),[\omega]_{*}\left(z_{0}\right)}\right) \\
& =\mathcal{S}\left(W_{y_{0}}^{[\omega]_{*}(z)} \circ q_{\omega}^{-1} \circ\left(W_{y_{0}}^{[\omega]_{*}\left(z_{0}\right)} \circ q_{\omega}^{-1}\right)^{-1}\right) \\
& =\mathcal{S}\left(P_{z}^{-1} \circ W_{[\bar{\omega}]_{*}^{-1}\left(y_{0}\right)}^{z} \circ\left(P^{-1} \circ W_{[\bar{\omega}]_{*}^{-1}\left(y_{0}\right)}^{z_{0}}\right)^{-1}\right) \\
& =\mathcal{S}\left(W_{[\bar{\omega}]_{*}^{-1}\left(y_{0}\right)}^{z} \circ\left(W_{[\bar{\omega}]_{*}^{-1}\left(y_{0}\right)}^{z_{0}}\right)^{-1} \circ P\right) \\
& =\mathcal{L}_{P}\left(\mathcal{S}\left(f_{[\bar{\omega}]_{*}^{-1}\left(y_{0}\right)}^{z, z_{0}}\right)\right)=\mathcal{L}_{P} \circ \beta_{[\bar{\omega}]_{*}^{-1}\left(y_{0}\right)}^{z_{0}}(z) .
\end{aligned}
$$

11.2. Quasiconformal extension of modular transformations. To give a proof of Theorem 3 (Modular transformation has qc-extension), we fix notation and definitions used in the proof

Notice that since Teichmüller space is biholomorphic to a bounded domain of $\mathbb{C}$, the cotangent space

$$
\mathcal{T}^{*}=\left\{(y, \varphi) \mid y \in \operatorname{Teich}(\bar{\Sigma}), \varphi \in Q\left(z_{0}, y\right)\right\}
$$

on Teich $(\bar{\Sigma})$ is holomorphically trivial by a theorem of Grauert (cf. e.g. Section 9 (D) of $[8]$ ).

Definition 16 (linear mapping $\ell_{y}$ ). Define a linear mapping from $Q_{-4}\left(z_{0}, y\right)$ to $\mathbb{C}$ by a trivialization of $\mathcal{T}^{*}$ :

$$
\mathcal{T}^{*} \ni(y, \varphi) \mapsto\left(y, \ell_{y}(\varphi)\right) \in \operatorname{Teich}(\bar{\Sigma}) \times \mathbb{C} .
$$

\subsection{Quasiconformal extension. First we prove}

Theorem 17. Any two Bers slices are quasiconformally equivalent. More precisely, for any $\left(z^{\prime}, y\right) \in \operatorname{Teich}(\Sigma) \times \operatorname{Teich}(\bar{\Sigma})$, there exists a quasiconformal mapping $h_{y}^{z^{\prime}}$ of $Q_{-4}\left(z_{0}, y_{0}\right)$ to $Q_{-4}\left(z^{\prime}, y\right)$ such that

$$
h_{y}^{z^{\prime}} \circ \beta_{y_{0}}^{z_{0}}(z)=\beta_{y}^{z^{\prime}}(z)
$$

for all $z \in \operatorname{Teich}(\Sigma)$. Furthermore, the dilatation of $h_{y}^{z^{\prime}}$ is $\exp \left\{d_{\text {Teich }(\bar{\Sigma})}\left(y, y_{0}\right)\right\}$.

Proof. Let $z \in \operatorname{Teich}(\Sigma)$. Put $c_{z}=\ell_{y_{0}} \circ \beta_{y_{0}}^{z_{0}}(z) \in \mathbb{C}$ and $A=\left\{c_{z} \mid z \in \operatorname{Teich}(\Sigma)\right\} \cup$ $\{\infty\}$. Then we define the mapping $H$ from Teich $(\bar{\Sigma}) \times A$ to $\hat{\mathbb{C}}$ by

$$
H(y, c)= \begin{cases}\ell_{y} \circ \beta_{y}^{z_{0}}(z) & \text { if } c=c_{z}, z \in \operatorname{Teich}(\bar{\Sigma}), \\ \infty & \text { if } c=\infty\end{cases}
$$

See Figure 12. We check here that $H$ is a labeled holomorphic motion of $A$ over $\operatorname{Teich}(\bar{\Sigma})$ with distinguished point $y_{0}$. Indeed, by definition, $H\left(y_{0}, c_{z}\right)=\ell_{y_{0}} \circ$ $\beta_{y_{0}}^{z_{0}}(z)=c_{z}$. Fix $y \in \operatorname{Teich}(\bar{\Sigma})$. Since $\beta_{y}^{z_{0}}$ is injective and $\beta_{y}^{z_{0}}(z) \neq \infty$, for $z \in$ Teich $(\Sigma), H(y, c)$ is also injective. From a theorem of Hejhal,

$$
H\left(y, c_{z}\right)=\ell_{y} \circ \beta_{y}^{z_{0}}(z)
$$

is holomorphic for $y \in \operatorname{Teich}(\bar{\Sigma})$ and fixed $z \in \operatorname{Teich}(\Sigma)$. Finally, we note that $H(y, \infty) \equiv \infty$ is also holomorphic on $y \in \operatorname{Teich}(\bar{\Sigma})$. 


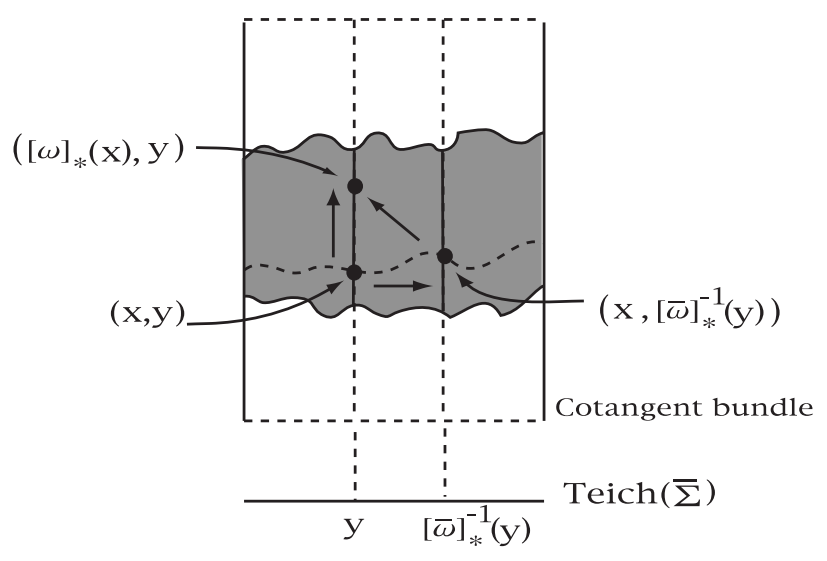

Figure 12. Process of qc extension: The vertical direction means the action on a slice via $[\omega]_{*}$, which is (essentially) factorized into two parts. One is the horizontal part represented by a qc map arising by application of the lambda lemma (Theorem 17). The other is a diagonal part, which is the linear map $\mathcal{L}_{P}$.

Therefore, the Improved $\lambda$-lemma tells us that $H$ can be extend as a labeled holomorphic motion $\hat{H}$ of $\hat{\mathbb{C}}$ over Teich $(\bar{\Sigma})$ with distinguished point $y_{0}$. Now, we define a quasiconformal mapping $h_{y}^{z^{\prime}}$ of $Q_{-4}\left(z_{0}, y_{0}\right)$ to $Q_{-4}\left(z^{\prime}, y\right)$ by

$$
h_{y}^{z^{\prime}}(\varphi)=\mathcal{L}_{y}^{z^{\prime}, z_{0}} \circ\left(\ell_{y}\right)^{-1} \circ \hat{H}\left(y, \ell_{y_{0}}(\varphi)\right) .
$$

Then $h_{y}^{z^{\prime}}$ satisfies the equation in the statement of this theorem. Indeed,

$$
\begin{aligned}
h_{y}^{z^{\prime}} \circ \beta_{y_{0}}^{z_{0}}(z) & =\mathcal{L}_{y}^{z^{\prime}, z_{0}} \circ\left(\ell_{y}\right)^{-1} \circ \hat{H}\left(y, \ell_{y_{0}} \circ \beta_{y_{0}}^{z_{0}}(z)\right) \\
& =\mathcal{L}_{y}^{z^{\prime}, z_{0}} \circ\left(\ell_{y}\right)^{-1} \circ \hat{H}\left(y, c_{z}\right) \\
& =\mathcal{L}_{y}^{z^{\prime}, z_{0}} \circ\left(\ell_{y}\right)^{-1} \circ \ell_{y} \circ \beta_{y}^{z_{0}}(z) \\
& =\mathcal{L}_{y}^{z^{\prime}, z_{0}} \circ \beta_{y}^{z_{0}}(z)=\beta_{y}^{z^{\prime}}(z) .
\end{aligned}
$$

By the Improved $\lambda$-lemma again, the dilatation of $h_{y}^{z^{\prime}}$ is $\exp \left\{d_{\operatorname{Teich}(\bar{\Sigma})}\left(y, y_{0}\right)\right\}$, since all $\mathcal{L}_{y}^{z^{\prime}, z_{0}}, \ell_{y}$, and $\ell_{y_{0}}$ are affine mappings.

Now, we restate and prove Theorem 3

Theorem 3, For $[\omega] \in \operatorname{Mod}(\Sigma)$, there is a $K\left([\omega], y_{0}\right)$-quasiconformal mapping $h_{\omega}$ of $Q_{-4}\left(z_{0}, y_{0}\right)$ onto itself such that

$$
h_{\omega} \circ \beta_{y_{0}}^{z_{0}}(z)=\beta_{y_{0}}^{z_{0}} \circ[\omega]_{*}(z), \text { for } z \in \operatorname{Teich}(\Sigma),
$$

where $K\left([\omega], y_{0}\right)=\exp \left\{d_{\text {Teich }(\bar{\Sigma})}\left(y_{0},[\bar{\omega}]_{*}\left(y_{0}\right)\right)\right\}$.

Proof. This is proved by the argument parallel to that of the proof of Theorem 17

The later part of the proof is the conclusion of the Improved $\lambda$-lemma. Let us prove the first part. Define a quasiconformal mapping $h_{\omega}$ by

$$
h_{\omega}(\varphi)=L_{y_{0}}^{z_{0},[\omega]_{*}\left(z_{0}\right)} \circ \mathcal{L}_{P} \circ\left(\ell_{[\bar{\omega}]_{*}^{-1}\left(y_{0}\right)}\right)^{-1} \circ \hat{H}\left([\bar{\omega}]_{*}^{-1}\left(y_{0}\right), \ell_{y_{0}}(\varphi)\right)
$$


for $\varphi \in Q_{-4}\left(z_{0}, y_{0}\right)$, where $P$ and $\mathcal{L}_{P}$ are taken and defined as in (3) of Proposition 11.1. Then $h_{\omega}$ satisfies the condition in the assertion. Indeed, since $\hat{H}\left([\bar{\omega}]_{*}^{-1}\left(y_{0}\right), \cdot\right)$ is $K\left([\omega], y_{0}\right)$-quasiconformal and all $L_{y_{0}}^{z_{0},[\omega]_{*}\left(z_{0}\right)}, \ell_{[\bar{\omega}]_{*}^{-1}\left(y_{0}\right)}, \mathcal{L}_{P}$, and $\ell_{y_{0}}$ are affine, $h_{\omega}$ is also $K\left([\omega], y_{0}\right)$-quasiconformal. In addition, for $z \in \operatorname{Teich}(\Sigma)$,

$$
\begin{aligned}
& h_{\omega} \circ \beta_{y_{0}}^{z_{0}}(z)=L_{y_{0}}^{z_{0},[\omega]_{*}\left(z_{0}\right)} \circ \mathcal{L}_{P} \circ\left(\ell_{[\bar{\omega}]_{*}^{-1}\left(y_{0}\right)}\right)^{-1} \circ H\left([\bar{\omega}]_{*}^{-1}\left(y_{0}\right), c_{z}\right) \\
& =L_{y_{0}}^{z_{0},[\omega]_{*}\left(z_{0}\right)} \circ \mathcal{L}_{P} \circ \beta_{[\bar{\omega}]_{*}^{-1}\left(y_{0}\right)}^{z_{0}}(z) \\
& =L_{y_{0}}^{z_{0},[\omega]_{*}\left(z_{0}\right)} \circ \beta_{y_{0}}^{[\omega]_{*}\left(z_{0}\right)} \circ[\omega]_{*}(z)=\beta_{y_{0}}^{z_{0}} \circ[\omega]_{*}(z) .
\end{aligned}
$$

Remark 6. By definition, the constant $K\left([\omega], y_{0}\right)$ coincides with the maximal dilatation of the extremal quasiconformal self-mapping of $S_{0}$ which is homotopic to $g_{0} \circ \bar{\omega} \circ g_{0}^{-1}$. Equivalently, let $\varphi_{0}$ be the point in its Bers slice corresponding to the Fuchsian group. Then $\log K\left([\omega], y_{0}\right)$ equals the hyperbolic distance between $\varphi_{0}$ and its image under $[\omega]_{*}$.

Since the boundary of Bers slices moves holomorphically on the base surfaces, we have (cf. [3]).

Corollary 8. The Hausdorff dimension of the complex boundary of Bers slices varies continuously with the complex structures of the base surfaces.

11.4. Similarity at cusps. Recall that a point $\zeta$ in a measurable set $\Omega \subset \mathbb{C}$ is said to be a $\delta$-measurable deep point, $\delta>0$ if

$$
\operatorname{area}(B(\zeta, r)-\Omega)=O\left(r^{2+\delta}\right)
$$

where $B(\zeta, r)$ is a disk of center $\zeta$ with radius $r$ (cf. [43]).

A quasiconformal mapping $\phi$ of $\mathbb{C}$ is said to be $C^{1+\alpha}$-conformal at $\zeta \in \mathbb{C}$ if $\phi^{\prime}(\zeta)$ exists and

$$
\phi(\zeta+t)=\phi(\zeta)+\phi^{\prime}(\zeta) t+O\left(|t|^{1+\alpha}\right) .
$$

Notice that any $C^{1+\alpha}$-conformal mapping $\phi$ at $\zeta$ is also $C^{1+\alpha^{\prime}}$-conformal at $\zeta$ for all $0<\alpha^{\prime}<\alpha$. Furthermore, we note that for two quasiconformal mappings, $\phi_{1}$ and $\phi_{2}$ on $\mathbb{C}$, if $\phi_{1}$ (resp. $\left.\phi_{2}\right)$ is $C^{1+\alpha_{1}}$ (resp. $C^{1+\alpha_{2}}$ )-conformal at $\zeta \in \mathbb{C}$ (resp. at $\left.\phi_{1}(\zeta)\right)$, then $\phi_{2} \circ \phi_{1}$ is $C^{1+\alpha}$-conformal at $\zeta$, where $\alpha=\min \left\{\alpha_{1}, \alpha_{2}\right\}$.

In [43, McMullen showed

Theorem 18 (Theorem 2.25 of [43]). Let $E$ be a measurable set on $\mathbb{C}$ and let $x \in \mathbb{C}$ be a $\delta$-measurable deep point. Then any quasiconformal mapping with vanishing Beltrami differential on $E$ is $C^{1+\alpha}$-conformal at $\zeta$, where $\alpha$ is dependent only on $\delta$ and the maximal dilatation of a given quasiconformal mapping.

We restate and prove a corollary of Theorem 3 in the Introduction:

Corollary 4 (All rational boundary points are similar to each other). There exists $\alpha>0$ that depends only on the base surface of a given Bers slice such that every Teichmüller modular transformation acting on a given Bers slice is $C^{1+\alpha}$-conformal at all rational boundary points.

Proof. A simple calculation shows any rational boundary point is a 1/2-measurable deep point for a given Bers slice by Theorem 1. Since every Teichmüller modular transformation acts on a Bers slice holomorphically, any quasiconformal extension of Teichmüller transformation is $C^{1+\alpha}$-conformal by Theorem[18. 
Next we choose $\alpha>0$ to be dependent only on the base surface. Let $\left[\omega_{1}\right]_{*}$ and $\left[\omega_{2}\right]_{*}$ be generators of the Teichmüller modular transformation acting on a given Bers slice. Suppose that $\left[\omega_{i}\right]_{*}$ is $C^{1+\alpha_{i}}$-conformal at each rational boundary point. Since the deepness of each rational boundary point is a universal constant $1 / 2$, by Remark [6, the constant $\alpha_{i}$ depends only on the dilatation of the Teichmüller mapping acting on the base surface of a given Bers slice representing $\left[\omega_{i}\right]_{*}$.

Let $\alpha$ be the supreme of $\min \left\{\alpha_{1}, \alpha_{2}\right\} / 2$ for all generators $\left\{\left[\omega_{1}\right]_{*},\left[\omega_{2}\right]_{*}\right\}$. Then $\alpha>0$ depends only on the base surface by definition and has the property in the assertion.

By using the same argument, we have

Corollary (All rational boundary points are similar). The quasiconformal map between two Bers slices given in Theorem 17 is $C^{1+\alpha}$-conformal at all rational boundary points for some $\alpha>0$.

\section{EXTENDED LOCAL PLEATING THEOREM}

In this section, applying Theorem2, we will give an extension of the local pleating theorem given in L. Keen and C. Series' beautiful paper [26]. The local pleating theorem gives a complete description of the local behavior of pleating varieties in quasifuchsian space.

12.1. Pleating varieties, local and limit pleating theorem. This section treats the definition of the pleating varieties in quasifuchsian groups after Keen and Series [26], and gives the statement of the local pleating theorem.

Let $\mathcal{F}$ be fuchsian space in $\mathcal{Q F}$. A component of the boundary of the convex core of the manifold corresponding to $[\eta]$ is said to be positive if it faces the positive end of $[\eta]$. The other component is called negative. Each component is a pleated surface in the sense of Thurston. We denote by $p l^{+}([\eta])$ (resp. $\left.p l^{-}([\eta])\right)$ the bending lamination of the positive (resp. negative) component.

Denote by $\mathcal{M L}$ and $\mathcal{P} \mathcal{M L}$ the space of measured laminations and projective measured laminations on $\Sigma$, respectively. For $\left[\nu_{1}\right],\left[\nu_{2}\right] \in \mathcal{P} \mathcal{M L}$, we define

$$
\begin{aligned}
\mathcal{P} \mathcal{L}_{\nu_{1}}^{ \pm} & =\left\{[\eta] \in \mathcal{Q F}-\mathcal{F} \mid\left[p l^{ \pm}([\eta])\right]=\left[\nu_{1}\right]\right\} ; \\
\mathcal{P} \mathcal{L}_{\nu_{1}, \nu_{2}} & =\mathcal{P} \mathcal{L}_{\nu_{1}}^{+} \cap \mathcal{P} \mathcal{L}_{\nu_{2}}^{-} .
\end{aligned}
$$

We call $\mathcal{P} \mathcal{L}_{\nu_{1}}^{ \pm}$the $\left[\nu_{1}\right]$-pleating varieties and $\mathcal{P} \mathcal{L}_{\nu_{1}, \nu_{2}}$ the $\left(\left[\nu_{1}\right],\left[\nu_{2}\right]\right)$-pleating plane. The connection between this notion and ours can be found from the following equation: Let $\nu_{1}$ be a rational lamination whose support is homotopic to $\mathbf{w}(\mathfrak{q})$. For $\mathcal{X}=\mathcal{B}_{G}$ or $\mathcal{E}$,

$$
\mathcal{P} \mathcal{L}_{\nu_{1}}^{+} \cap \mathcal{X}=\mathcal{P}_{\mathfrak{q}}^{\mathcal{X}} .
$$

In [26], L. Keen and C. Series proved the following theorem:

Theorem 19 (Non-singularity of pleating varieties). Let $p / q, p^{\prime} / q^{\prime} \in \hat{\mathbb{Q}}$ with $p / q \neq$ $p^{\prime} / q^{\prime}$. Let $\nu_{1}$ and $\nu_{2}$ be rational measured laminations whose supports are homotopic to $\mathbf{w}(p / q)$ and $\mathbf{w}\left(p^{\prime} / q^{\prime}\right)$ respectively. Then $\mathcal{P} \mathcal{L}_{\nu_{1}, \nu_{2}}$ and $\mathcal{P} \mathcal{L}_{\nu_{2}, \nu_{1}}$ are the components of the preimage of $\mathbb{R}_{>0} \times \mathbb{R}_{>0}$ under the mapping:

$$
\mathcal{Q F}-\mathcal{F} \ni[\eta] \mapsto\left(\operatorname{tr}^{2} \eta(\mathbf{w}(p / q)), \operatorname{tr}^{2} \eta\left(\mathbf{w}\left(p^{\prime} / q^{\prime}\right)\right)\right) .
$$


Furthermore, this mapping is non-singular on $\mathcal{P} \mathcal{L}_{\nu_{1}, \nu_{2}} \cup \mathcal{P} \mathcal{L}_{\nu_{2}, \nu_{1}}$ and the boundary of $\mathcal{P} \mathcal{L}_{\nu_{1}, \nu_{2}} \cup \mathcal{P} \mathcal{L}_{\nu_{2}, \nu_{1}}$ can be computed by solving $\operatorname{tr}^{2} \eta(\mathbf{w}(p / q))=4$ and $\operatorname{tr}^{2} \eta\left(\mathbf{w}\left(p^{\prime} / q^{\prime}\right)\right)=$ 4 on this component.

Here we note that this theorem does not mention the non-singularity of their trace functions at the boundary of these pleating varieties, which is one of the main theorems of this section.

To prove this theorem, they showed useful results, called the Local pleating theorem and the Limit pleating theorem (Theorems 5.1 and 8.1 of [26]) which, for convenience, we explain below. For $\nu_{1} \in \mathcal{M L}$, we denote by $\lambda_{\nu_{1}}([\eta])$ the complex length of the lamination $\nu_{1}$ of the quasifuchsian manifold associated with $[\eta]$. By definition, if $\nu_{1}=t \gamma$ where $t>0$ and $\gamma$ is a simple closed curve on $\Sigma$, this is just $t$ times the complex length defined from the trace function of the corresponding simple closed curve. In the case of irrational laminations, we define the length function by the limiting process (cf. [26]). The real part of the complex length is nothing but the usual length of lamination $\nu_{1}$. The complex function $\lambda_{\nu_{1}}$ takes positive real values on $\mathcal{P} \mathcal{L}_{\nu_{1}}^{+} \cup \mathcal{F} \cup \mathcal{P} \mathcal{L}_{\nu_{1}}^{-}$.

Theorem (Local pleating theorem). Let $\nu_{1} \in \mathcal{M L}$. Every group $\left[\eta_{0}\right]$ in $\mathcal{P} \mathcal{L}_{\nu_{1}}^{+} \cup$ $\mathcal{F} \cup \mathcal{P} \mathcal{L}_{\nu_{1}}^{-}$has a neighborhood $U$ in $\mathcal{Q F}$ such that for $[\eta] \in U, \lambda_{\nu_{1}}([\eta]) \in \mathbb{R}$ implies $[\eta] \in \mathcal{P} \mathcal{L}_{\nu_{1}}^{+} \cup \mathcal{F} \cup \mathcal{P} \mathcal{L}_{\nu_{1}}^{-}$.

Theorem (Limit pleating theorem). Let $\nu_{1}$ and $\nu_{2}$ be mutually distinct rational laminations. Let $\left\{\left[\eta_{n}\right]\right\}_{n}$ be a sequence on $\mathcal{P} \mathcal{L}_{\nu_{1}, \nu_{2}}$. If $\operatorname{Re} \lambda_{\nu_{i}}\left(\left[\eta_{n}\right]\right) \rightarrow c_{i} \geq 0$ for each $i$, then $\left\{\left[\eta_{n}\right]\right\}_{n}$ contains a subsequence with algebraic limit $\left[\eta_{\infty}\right]$. Further, $\left[\eta_{\infty}\right]$ is quasifuchsian if and only if $c_{i}>0$ for $i=1,2$.

Actually, L. Keen and C. Series showed their theorems in more general situations. The theorems above are modified to simplify the argument here.

12.2. Extended local pleating theorem. Using notation given in the previous section, we restate Theorem 4 as follows: Recall that a geometrically finite boundary group is said to be of type $n(n=1,2)$ if it admits $n$ distinct conjugacy classes of accidental parabolic transformations.

Theorem 4 (Extended local pleating theorem). Let $\left[\eta_{0}\right]$ be a geometrically finite boundary group. Then there exists a neighborhood $\mathcal{U}$ of $\left[\eta_{0}\right]$ in $\mathcal{R}$ so that one of the following holds:

1. Suppose that $\left[\eta_{0}\right]$ is of type 1 . Take $p / q \in \mathbb{Q}$ such that $\mathbf{w}(p / q)$ corresponds to the accidental parabolic of $\left[\eta_{0}\right]$. Let $\nu_{1}$ be a measured lamination whose support is homotopic to $\mathbf{w}(p / q)$. Then either $\mathcal{U} \cap \mathcal{P} \mathcal{L}_{\nu_{1}}^{+}$or $\mathcal{U} \cap \mathcal{P} \mathcal{L}_{\nu_{1}}^{-}$is the preimage under the holomorphic mapping

$$
\mathcal{U} \ni[\eta] \mapsto \operatorname{tr}^{2} \eta(\mathbf{w}(p / q))
$$

of $\mathbb{R}_{>4}=\{t \in \mathbb{R} \mid r>4\}$. Furthermore, if $\mathcal{U} \cap \mathcal{P} \mathcal{L}_{\nu_{1}}^{+}$is not empty, $\mathcal{U} \cap \mathcal{P} \mathcal{L}_{\nu_{1}}^{-}$ is empty and vice versa.

2. Otherwise, let $p / q, p^{\prime} / q^{\prime} \in \mathbb{Q}$ correspond to the accidental parabolics of $\left[\eta_{0}\right]$. Let $\nu_{1}$ and $\nu_{2}$ be measured laminations whose supports are homotopic to $\mathbf{w}(p / q)$ and $\mathbf{w}\left(p^{\prime} / q^{\prime}\right)$ respectively. Then $\mathcal{U} \cap \mathcal{P} \mathcal{L}_{\nu_{1}, \nu_{2}}$ is the preimage of the mapping

$$
\mathcal{U} \ni[\eta] \mapsto\left(\operatorname{tr}^{2} \eta(\mathbf{w}(p / q)), \operatorname{tr}^{2} \eta\left(\mathbf{w}\left(p^{\prime} / q^{\prime}\right)\right)\right)
$$

of $\mathbb{R}_{>4} \times \mathbb{R}_{>4}$. 
Furthermore, in all cases, the preimages of these mappings are connected and contractible.

Proof. We first prove the case of the type 2 geometrically finite boundary group. From Proposition 10.2, the differential of the mapping

$$
\Psi_{0}([\eta])=\left(\operatorname{tr}^{2} \eta(\mathbf{w}(p / q)), \operatorname{tr}^{2} \eta\left(\mathbf{w}\left(p^{\prime} / q^{\prime}\right)\right)\right)
$$

has maximal rank at $\left[\eta_{0}\right]$. Hence there exists a neighborhood $\mathcal{U}$ of $\left[\eta_{0}\right]$ so that $\Psi_{0}$ is biholomorphic on $\mathcal{U}, \mathcal{U}$ does not intersect the fuchsian slice, and $\Psi_{0}(\mathcal{U})$ is a polydisk with center $(4,4)$ and radius $r_{0}>0$. Put $N_{2}=\left\{(t, s) \in \mathbb{R} \mid 4<t, s<4+r_{0}\right\}$. Since $\left[\eta_{0}\right] \in \overline{\mathcal{P} \mathcal{L}_{\nu_{1}, \nu_{2}}}, \mathcal{U} \cap \mathcal{P} \mathcal{L}_{\nu_{1}, \nu_{2}}$ is not empty and contained in $\Psi_{0}^{-1}\left(N_{2}\right)$. By virtue of the local and limit pleating theorem, $\mathcal{U} \cap \mathcal{P} \mathcal{L}_{\nu_{1}, \nu_{2}}$ is open and closed in $\Psi_{0}^{-1}(P)$. Therefore

$$
\mathcal{U} \cap \mathcal{P} \mathcal{L}_{\nu_{1}, \nu_{2}}=\left\{[\eta] \in \mathcal{U} \mid 4<\operatorname{tr}^{2} \eta(\mathbf{w}(p / q)), \operatorname{tr}^{2} \eta\left(\mathbf{w}\left(p^{\prime} / q^{\prime}\right)\right)<4+r_{0}\right\} .
$$

Next we assume that $\left[\eta_{0}\right]$ is of type 1 . The space of representations $\mathcal{R}$ has anticonformal involution which is induced from the complex conjugation $\zeta \mapsto \bar{\zeta}$ on the phase space $\hat{\mathbb{C}}$. Since the ends of each Kleinian manifold are switched by this involution, we may suppose that the accidental parabolic of $\left[\eta_{0}\right]$ corresponds to the positive end invariant of $\left[\eta_{0}\right]$; that is, $\nu_{+}\left(\left[\eta_{0}\right]\right) \in \hat{\mathbb{Q}}$ and $\nu_{-}\left(\left[\eta_{0}\right]\right) \in \mathbb{H}$. Therefore $G_{0}:=\eta_{0}\left(\pi_{1}\right)$ has the invariant component, which is no longer distinguished. Let $y_{0}=\left(g_{0}, S_{0}\right) \in \operatorname{Teich}(\bar{\Sigma})$ be the conjugate of the point of Teich $(\Sigma)$ corresponding to $\nu_{-}\left(\left[\eta_{0}\right]\right) \in \mathbb{H}$.

Here, we use the notation given in the previous section frequently. From the argument above, $\left[\eta_{0}\right]$ is on the image by the holonomy map $\mathcal{T}^{*} \rightarrow \mathcal{R}$ of the boundary of the Bers slice $\beta_{y_{0}}^{z_{0}}(\operatorname{Teich}(\bar{\Sigma}))$. Since the holonomy map is a local analytic homeomorphism, there exists a neighborhood $\mathcal{U}^{\prime}$ of $\left[\eta_{0}\right]$ in $\mathcal{R}$ and a holomorphic mapping proj, which is induced from the projection of the cotangent bundle to Teichmüller space, from $\mathcal{U}^{\prime}$ to $\operatorname{Teich}(\bar{\Sigma})$ so that $\operatorname{proj}\left(\left[\eta_{0}\right]\right)=y_{0}$ and any $[\eta] \in \mathcal{U}^{\prime}$ is induced from the projective structure whose underlying complex structure associates with $\operatorname{proj}([\eta])$.

We define the holomorphic mapping $\Psi_{0}$ on $\mathcal{U}^{\prime}$ by

$$
\Psi_{0}: \mathcal{U}^{\prime} \ni[\eta] \mapsto\left(\operatorname{proj}([\eta]), \operatorname{tr}^{2} \eta(\mathbf{w}(p / q))\right) \in \operatorname{Teich}(\bar{\Sigma}) \times \mathbb{C} .
$$

We claim the following:

Claim. $\Psi_{0}$ is biholomorphic on a neighborhood of $\left[\eta_{0}\right]$.

Indeed, let $F_{0}$ be a fuchsian group of type $(1,1)$ such that $\Lambda\left(F_{0}\right)=\mathbb{R}$ and satisfies $\mathbb{L} / F_{0}=S_{0}$. We consider the Bers slice whose center is $F_{0}$. As was said above, $\left[\rho_{x^{b}(p / q)}^{b}\right]=\left[\eta_{0}\right]$ and $\operatorname{proj}\left(\left[\rho_{\varphi}^{b}\right]\right)=y_{0}$ on some neighborhood of $x^{b}(p / q)$ in $Q_{-4}\left(F_{0}\right)$.

A quasiconformal deformation of $\eta_{0}$ induces a holomorphic section $s_{0}$ from a neighborhood $N_{0}$ of $y_{0}$ in Teich $(\bar{\Sigma})$ into $\mathcal{U}^{\prime}$ so that $s_{0}\left(y_{0}\right)=\left[\eta_{0}\right]$, proj $\circ s_{0}(y)=y$ and

$$
\operatorname{tr}^{2} s_{0}(y)(\mathbf{w}(p / q)) \equiv 4
$$

on $N_{0}$. Together with Theorem 2 we conclude the claim.

Let us continue to prove Theorem 4 . Shrink the neighborhood $\mathcal{U}_{0}$ such that:

(1) $\Psi_{0}$ is biholomorphic on $\mathcal{U}_{0}$, 
(2) the image $\Psi_{0}\left(\mathcal{U}_{0}\right)$ is the product of a simply connected domain $\operatorname{proj}\left(\mathcal{U}_{0}\right)$ and a small disk with center 4 and radius $r_{0}$, and

(3) $\mathcal{U}_{0} \cap \mathcal{F}=\emptyset$.

Then we finally claim that

$$
\mathcal{U}_{0} \cap \mathcal{P} \mathcal{L}_{\nu_{1}}^{+}=\left\{[\eta] \in \mathcal{U}_{0} \mid \operatorname{tr}^{2} \eta(\mathbf{w}(p / q)) \in \mathbb{R}_{>4}\right\},
$$

for appropriate small constant $r_{0}>0$.

Indeed, on $\mathcal{P} \mathcal{L}_{\nu_{1}}^{+}$, the complex length of $\nu_{1}$ takes real values. Therefore, the left-hand side is contained in the right.

Let $[\eta] \in \Psi_{0}^{-1}\left(N_{1}\right)$ with $\operatorname{proj}([\eta])=y_{0}$. Let $\varphi \in Q_{-4}\left(F_{y_{0}}\right)$ so that $[\eta]=\left[\rho_{\varphi}^{b}\right]$. We know that the $p / q$-pleating ray is a connected component of the set

$$
\left\{\varphi \in Q_{-4}\left(F_{y_{0}}\right)-\{0\} \mid \operatorname{tr}^{2} \rho_{\varphi}^{b}(\mathbf{w}(p / q))>4\right\} .
$$

By Theorem 2, a set

$$
\left\{\varphi \in Q_{-4}\left(F_{y_{0}}\right) \mid \operatorname{tr}^{2} \rho_{\varphi}^{b}(\mathbf{w}(p / q)) \in \mathbb{R}\right\}
$$

is a real analytic curve near $x^{b}(p / q)$. Hence by taking $r_{0}$ sufficiently small, for $\varphi \in$ $Q_{-4}\left(F_{y_{0}}\right)$, if $\left[\rho_{\varphi}^{b}\right] \in \Psi_{0}^{-1}\left(N_{1}\right), \varphi$ lies on the $p / q$-pleating ray in $\mathcal{B}_{F_{y_{0}}}$. Since $\mathcal{U}_{0}$ does not intersect the fuchsian locus, applying the Local pleating theorem and the Limit pleating theorem, the set consisting of $[\eta] \in \Psi_{0}^{-1}\left(N_{1}\right) \cap \mathcal{P} \mathcal{L}_{\nu_{1}}^{+}$is open and closed in $\Psi_{0}^{-1}\left(N_{1}\right)$. This means that $[\eta] \in \mathcal{P} \mathcal{L}_{\nu_{1}}^{+}$for $[\eta] \in \mathcal{U}_{0}$ with $4<\operatorname{tr}^{2} \eta(\mathbf{w}(p / q))<4+r_{0}$. Thus, we may let $\mathcal{U}$ be the resulting neighborhood.

\section{Appendix A. Relation to Complex dynamics}

A similarity phenomenon, Corollary 4 on Bers slices gives columns in the dictionary between rational maps and Kleinian groups. In particular, this corresponds to the similarity of parabolic points of the Mandelbrot set $M$ via tuning. The tuning is a homeomorphism from the Mandelbrot set to its copy in itself defined by Renormalization (cf. e.g. Section 5 of [38]).

We will not give the concrete definition of the notion in complex dynamics, e.g. tuning and a little Mandelbrot copy, and so forth. However we should know here the following four properties:

(1) There are two kinds of Mandelbrot copies: primitive copies and satellite copies (cf. e.g. [46]).

(2) If a Mandelbrot copy is primitive, the corresponding tuning is extended quasiconformally to the whole complex plane. If a copy is satellite, the tuning is quasiconformal at $M-\{1 / 4\}$ (Theorem 5.5 of [38]).

(3) Every parabolic point is a 1/2-deep point for the interior of the Mandelbrot set (cf. e.g. Lemmas 6.1 and 6.2 of [46]).

(4) A tuning is holomorphic on the interior of the Mandelbrot set (cf. Theorem 2 of [12]).

Applying (2), (3), and (4) together with Theorem 18, we have

Theorem 20. Let $M$ be the Mandelbrot set and $M_{c}$ a Mandelbrot copy. Then the tuning from $M$ to $M_{c}$ is $C^{1+\alpha}$-conformal at every parabolic point except for $1 / 4 \in \partial M$. If $M_{c}$ is primitive, the tuning is also $C^{1+\alpha}$-conformal at $1 / 4$. 
Thus we obtain the following table:

\begin{tabular}{|l|l|}
\hline Once puncture torus groups & Quadratic polynomials \\
\hline \hline Bers slice $\mathcal{B}_{G}$ & Mandelbrot set $M$ \\
\hline Rational boundary point & Parabolic point \\
\hline $\begin{array}{l}\text { Rational boundary points are } \\
\text { inward-pointing cusps (Theorem } \\
1 \text { 1) }\end{array}$ & $\begin{array}{l}\text { Root of a primitive Mandelbrot } \\
\text { copy is an inward-pointing cusp } \\
\text { (cf. e.g. Lemma 6.2 of [46] })\end{array}$ \\
\hline \hline $\begin{array}{l}\text { Rational boundary points are 1/2- } \\
\text { deep points (Theorem 1) }\end{array}$ & $\begin{array}{l}\text { Parabolic points are 1/2-deep } \\
\text { points }\end{array}$ \\
\hline $\begin{array}{l}\text { Teichmüller modular transforma- } \\
\text { tion acting on a Bers slice }\end{array}$ & Tuning \\
\hline $\begin{array}{l}\text { Teichmüller modular transforma- } \\
\text { tion has qc-extension (Theorem } 3 \text { ) }\end{array}$ & $\begin{array}{l}\text { Tuning has qc-extension (locally) } \\
\text { (M. Lyubich) }\end{array}$ \\
\hline $\begin{array}{l}\text { Teichmüller modular transforma- } \\
\text { tion is } C^{1+\alpha} \text {-conformal at rational } \\
\text { boundary points (Corollary 4]) }\end{array}$ & $\begin{array}{l}\text { Tuning is } C^{1+\alpha} \text {-conformal at par- } \\
\text { abolic points (Theorem [20) }\end{array}$ \\
\hline
\end{tabular}

Many people observed some similarity phenomena in the theory of complex dynamics (cf. e.g. [35], 38], [55], and [56]).

Acknowledgment. The author is very grateful to his adviser, Professor Yoichi Imayoshi, for his constant encouragement and advice. The author thanks Dr. Yohei Komori for his helpful conversations and inspiration.

The author also expresses his gratitude to Professor Caroline Series for her favorable advice and comments on this work and for her interesting lectures on pleating coordinates at the Osaka City University in July 1998 and at the Nara Women's University in January 2000. Her lecture invited him to the study of analytic and geometric properties of several slices of once punctured torus groups. The author thanks Professor Curtis T. McMullen and Professor David J. Wright for telling him about strange phenomena on the boundary of the Maskit slice, and thanks Mr. Peter Liepa, Professor D. J. Wright, and Professor Yasushi Yamashita for permission to include their figures here.

Almost all of the complex dynamics section (Appendix A) was completed during the author's visit to SUNY at Stony Brook from May 2000 to February 2001. The author is grateful to Professor Yair Minsky and Professor Mikhail Lyubich for their helpful conversations and advice. He also thanks the Department of Mathematics of SUNY at Stony Brook for their hospitality. Finally, he thanks the referee for his/her useful comments and for pointing out various errors in the previous version of this paper.

\section{REFERENCES}

[1] L. V. Ahlfors, Complex Analysis, 3rd ed., McGraw-Hill, 1979. MR 80c:30001

[2] L. V. Ahlfors and L. Bers, Riemann mapping theorem for variable metrics, Ann. of Math. 72 (1960), 385-404. MR 22:5813

[3] K. Astala, Area distortion of quasiconformal mappings, Acta Math. 173 (1994), 37-60. MR 95m:30028b 
[4] L. Bers, A non-standard integral equation with applications to quasiconformal mappings, Acta Math. 116 (1966), 113-134. MR 33:273

[5] $\_$, On boundaries of Teichmüller spaces and on Kleinian groups, Ann. of Math. 91 (1970), 570-600. MR 45:7044

[6] L Fiber spaces over Teichmüller spaces, Acta Math. 130 (1973), 86-126. MR 55:3323

[7] $ـ$, The action of the modular group on the complex boundary Riemann surfaces and related topics (I. Kra and B. Maskit, eds.), Ann. of Math. Studies 97 (1981), 33-53, Princeton University Press. MR 83b:32019

[8] - Finite dimensional Teichmüller spaces and generalizations, Bull. Amer. Math. Soc. (N.S.) 5 (1981), 131-172. MR 82k:32050

[9] L. Bers and H. L. Royden, Holomorphic families and injections, Acta Math. 157 (1986), 259-286. MR 88i:30034

[10] F. Bonahon, Bouts des variétés hyperboliques de dimension 3, Ann. of Math. 124, 71-158. MR 88c:57013

[11] P. Buser, Geometry and spectra of compact Riemann surfaces, Birhäuser Boston, Inc., Boston, MA, 1992. MR 93g:58149

[12] A. Douady and J. H. Hubbard, On the dynamics of polynomial-like maps, Ann. Sci. École Norm. Sup. 18 (1985), 287-343. MR 87f:58083

[13] C. J. Earle, Some intrinsic coordinates on Teichmüller space, Proc. Amer. Math. Soc. 83 (1981), 527-531. MR 82j:32050

[14] C. J. Earle, I. Kra, and S. L. Krushkal, Holomorphic motions and Teichmüller spaces, Trans. Amer. Math. Soc. 343 (1994), 927-948. MR 94h:32035

[15] D.B.A. Epstein and A. Marden, Convex hulls in hyperbolic space, a theorem of Sullivan, and measured pleated surfaces. Analytic and Geometric Aspects of Hyperbolic Space (D.B.A Epstein ed.), London Math. Soc. Lecture Notes 111 112-253, Cambridge Univ. Press, Cambridge, 1987. MR 89c:52014

[16] F. P. Gardiner, Schiffer's interior variation and quasiconformal mappings, Duke Math. J. 42 (1975), 371-380. MR 52:3519

[17] F. W. Gehring, Characteristic properties of quasidisks, Séminaire de Mathématiques Supérieures [Seminar on Higher Mathematics], 84. Presses de L'université de Montreal, Montreal, Que., 1982. MR 84a:30036

[18] F. W. Gehring and G. J. Martin, Iteration theory and inequalities for Kleinian groups, Bull. Amer. Math. Soc. 21 (1989), 57-63. MR 90k:30086

[19] G. H. Hardy and E. M. Wright, An introduction to the theory of numbers. 5th edition, Clarendon Press, Oxford (1979). MR 81i:10002

[20] D. A. Hejhal, Monodromy groups and linearly polymorphic functions, Acta Math. 135 (1975), 1-55. MR 57:3380

[21] Y. Imayoshi and M. Taniguchi, An introduction to Teichmüller spaces, Springer-Verlag (1992) MR 94b:32031

[22] K. Ito, Exotic projective structures and boundaries of quasi-Fuchsian space, Duke Math. J. 105 (2000), 185-209. MR 2001j:30038

[23] T. Jørgensen, On pair of once punctured tori, Preprint.

[24] L. Keen and C. Series, Pleating coordinates for the Maskit embedding of the Teichmüller space of punctured tori, Topology 32 (1993), 719-749. MR 95g:32030

[25] $\ldots$, The Riley slice of Schottky space, Proc. London Math. Soc. (3) 69 (1994), 72-90. MR 95j:32033

[26] Pleating invariants for punctured torus groups, to appear in Topology.

[27] L. Keen, B. Maskit, and C. Series, Geometric finiteness and uniqueness for Kleinian groups with circle packing limit sets, J. Reine. Angew. Math. 436 (1993), 209-219. MR 94b:30053

[28] S. Kerckhoff and W. Thurston, Noncontinuity of the action of the modular group at Bers' boundary of Teichmüller space, Invent. Math. 100 (1990), 25-48. MR 91a:57038

[29] Y. Komori, Minsky's pivot theorem and its application to the Earle slice of punctured torus groups. Hyperbolic spaces and related topics (S. Kamiya, ed.), RIMS, Kokyouroku, Kyoto Univ. 110 (1999), 91-102.

[30] Y. Komori and C. Series, Pleating coordinates for the Earle embedding. Ann. Fac. Sci. Toulouse Math. (6) 10 (2001), 69-105.

[31] Y. Komori, T. Sugawa, M. Wada, and Y. Yamashita, Discreteness locus in projective structures, http://www.kusm.kyoto-u.ac.jp/complex/Bers/ 
[32] I. Kra, Nonvariational global coordinates for Teichmüller spaces. Holomorphic functions and Moduli, II (D. Drasin, C. J. Earle, F. W. Gehring, I. Kra, and A. Marden, eds.), SpringerVerlag: MSRI publications 10 (1988), 221-249. MR 90b:32044

[33] _ Horocyclic coordinates for Riemann surfaces and moduli spaces, I: Teichmüller and Riemann spaces of Kleinian groups. J. Amer. Math. Soc. 3 (1990), 499-578. MR 91c:32014

[34] I. Kra and B. Maskit, Bases on quadratic differentials, Comment. Math. Helv. 57 (1982), 603-626. MR 85b:30059

[35] T. Lei, Similarity between the Mandelbrot set and Julia sets, Commun. Math. Phys. 134 (1990), 587-617. MR 92k:58228

[36] O. Lehto, Univalent functions and Teichmüller spaces, Graduate Texts in Mathematics 109 (1986), Springer-Verlag. MR 88f:30073

[37] O. Lehto and K.I.Virtanen, Quasiconformal mapping in the plane. Second edition. SpringerVerlag, New York-Heidelberg, 1973. MR 49:9202

[38] M. Lyubich, Feigenbaum-Coullet-Tresser universality and Milnor's fairiness conjecture, Ann. of Math. 149 (1999), 319-420. MR 2000d:37051

[39] R. Mañé, P. Sad, and D. Sullivan, On the dynamics of rational maps, Ann. Sci. École. Norm. Sup. 16 (1983), 193-217. MR 85j:58089

[40] K. Matsuzaki and M. Taniguchi, Hyperbolic manifolds and Kleinian groups, Oxford Mathematical Monographs, The Clarendon Press, Oxford University Press, New York, 1998. MR 99g:30055

[41] C. T. McMullen, Families of rational maps and iterative root-finding algorithms, Ann. of Math. 125 (1987), 467-493. MR 88i:58082

[42] _ Cusps are dense, Ann. of Math. 133 (1991), 217-247. MR 88i:58082

[43] - Renormalization and 3-manifolds which fiber over the circle, Ann. of Math. Studies 142 (1996), Princeton University Press, Princeton, NJ. MR 97f:57022

[44] Complex earthquakes and Teichmüller theory, J. Amer. Math. Soc. 11 (1998), 283320. MR 98i:32030

[45] G. J. Martin, On the geometry of Kleinian groups. Quasiconformal mappings and analysis, 253-274 (P.L.Duren, J.M.Heinonen, B.G.Osgood, and B.P.Palka, eds.), A collection of papers honoring F. W. Gehring, Springer-Verlag, New York, 1998. MR 98k:30061

[46] J. Milnor, Periodic orbits, external rays and the Mandelbrot set: an expository account, 'Geometrie Complexe et Systemes Dynamiques', Astérisque 261 (2000) 277-333. MR 2002e:37067

[47] Y. N. Minsky, The classification of punctured torus groups, Ann. of Math. 149 (1999), 559626. MR 2000f:30028

[48] H. Miyachi, On the horocyclic coordinate for the Teichmüller space of once punctured tori, Proc. Amer. Math. Soc. 130 (2002), 1019-1029. MR 2002j:32010

[49] _ On cusps in the boundaries of the Earle slice and the Maskit slice for once punctured torus groups, preprint (1999).

[50] D. Mumford, C. T. McMullen, and D. J. Wright, Limit sets of free two-generator Kleinian groups, Preprint, Version 0.95 (1990).

[51] J. P. Otal, Sur le coeur convexe d'une variété hyperbolique de dimension 3, preprint.

[52] J. R. Parker and C. Series, Bending formulae for convex hull boundaries, J. Anal. Math. 64 (1995), 165-198. MR 97k:30057

[53] P.Liepa, http://reality.sgi.com/pliepa_tor/slices.html

[54] Ch. Pommerenke, Boundary behavior of conformal maps, Springer-Verlag (1992). MR 95b:30008

[55] J. Rivera, On the continuity of Hausdorff dimension of Julia sets and similarity between the Mandelbrot set and Julia sets, Fund. Math. 170, 287-317.

[56] _ Similarity between the Mandelbrot set and Julia sets and hairiness, preprint.

[57] H. Shiga, Projective structures on Riemann surfaces and Kleinian groups, J. Math. Kyoto. Univ. 27 (1987) 433-438. MR 88k:30056

[58] T. Sugawa, The Bers projections and the $\lambda$-lemma, J. Math. Kyoto. Univ. 32 (1992), 701-713. MR 93k:32043

[59] P. Susskind, Kleinian groups with intersecting limit sets, J.Analyse Math. 52 (1989), 26-38. MR 90f:57045

[60] D. Sullivan and W. Thurston, Extending holomorphic motions, Acta Math. 157 (1986), 243258. MR 88i:30033 
[61] Z. Słodkowski, Holomorphic motions and polynomial hulls, Proc. Amer. Math. Soc. 111 (1991), 347-355. MR 91f:58078

[62] M. Wada, OPTi3.0, http://vivaldi.ics.nara-wu.ac.jp/ ${ }^{\sim}$ wada/OPTi/

[63] D. J. Wright, The shape of the boundary of Maskit's embedding of the Teichmüller space of once punctured tori, Preprint, Version 0.8 (1988).

[64] - http://www.math.okstate.edu/ ${ }^{\sim}$ wrightd/

[65] Y. Yamashita, Pictures, http://vivaldi.ics.nara-wu.ac.jp/ yamasita/Slice/

Department of Mathematics, Osaka City University, Sumiyoshi, Osaka, 558-8585, JAPAN

E-mail address: miyaji@sci.osaka-cu.ac.jp

Current address: Graduate School of Science, Department of Mathematics, Osaka University, 1-1, Machikaneyama-cho, Toyonaka, Osaka, 560-0023, Japan

E-mail address: miyaji@gaia.math.wani.osaka-u.ac.jp 